\title{
THE EXTENDED VIRGO CLUSTER CATALOG
}

\author{
Suk Kim ${ }^{1}$, Soo-Chang Rey ${ }^{1,5}$, Helmut Jerjen ${ }^{2}$, Thorsten Lisker ${ }^{3}$, Eon-Chang Sung ${ }^{4}$, Youngdae Lee ${ }^{1}$, \\ Jiwon Chung ${ }^{1}$, Mina PaK ${ }^{1}$, Wonhyeong Yi ${ }^{1}$, AND Woong LeE ${ }^{1}$ \\ ${ }^{1}$ Department of Astronomy and Space Science, Chungnam National University, 99 Daehak-ro, Daejeon 305-764, Korea \\ ${ }^{2}$ Research School of Astronomy and Astrophysics, The Australian National University, Cotter Road, Weston, ACT 2611, Australia \\ ${ }^{3}$ Astronomisches Rechen-Institut, Zentrum für Astronomie der Universität Heidelberg (ZAH), Mönchhofstraße 12-14, D-69120 Heidelberg, Germany \\ ${ }^{4}$ Korea Astronomy \& Space Science institute, 776 Daedeokdae-ro, Daejeon 305-348, Korea \\ Received 2014 March 15; accepted 2014 September 8; published 2014 December 2
}

\begin{abstract}
We present a new catalog of galaxies in the wider region of the Virgo cluster, based on the Sloan Digital Sky Survey (SDSS) Data Release 7. The Extended Virgo Cluster Catalog (EVCC) covers an area of $725 \mathrm{deg}^{2}$ or $60.1 \mathrm{Mpc}^{2}$. It is 5.2 times larger than the footprint of the classical Virgo Cluster Catalog (VCC) and reaches out to 3.5 times the virial radius of the Virgo cluster. We selected 1324 spectroscopically targeted galaxies with radial velocities less than $3000 \mathrm{~km} \mathrm{~s}^{-1}$. In addition, 265 galaxies that have been overlooked in the SDSS spectroscopic survey but have available redshifts in the NASA Extragalactic Database are also included. Our selection process secured a total of 1589 galaxies, 676 of which are not included in the VCC. The certain and possible cluster members are defined by means of redshift comparison with a cluster infall model. We employed two independent and complementary galaxy classification schemes: the traditional morphological classification based on the visual inspection of optical images and a characterization of galaxies from their spectroscopic features. SDSS $u, g, r, i$, and $z$ passband photometry of all EVCC galaxies was performed using Source Extractor. We compare the EVCC galaxies with the VCC in terms of morphology, spatial distribution, and luminosity function. The EVCC defines a comprehensive galaxy sample covering a wider range in galaxy density that is significantly different from the inner region of the Virgo cluster. It will be the foundation for forthcoming galaxy evolution studies in the extended Virgo cluster region, complementing ongoing and planned Virgo cluster surveys at various wavelengths.
\end{abstract}

Key words: catalogs - galaxies: clusters: general - surveys

Online-only material: color figures, machine-readable tables

\section{INTRODUCTION}

The current theory of structure formation in the standard cold dark matter universe predicts that the build-up of galaxy clusters is characterized by the accretion of individual galaxies and groups. These small galaxy aggregates preferentially travel from the cosmic web along filamentary substructures to the densest regions (van Haarlem \& van de Weygaert 1993; Bond et al. 1996; Springel et al. 2005). Nearby galaxy clusters $\left(c z<10,000 \mathrm{~km} \mathrm{~s}^{-1}\right)$ and their surrounding regions represent the current endpoint of this evolution and occupy the apex of the process.

However, despite the importance of these extended cluster regions, most observational studies of nearby galaxy clusters have concentrated on the densest parts, the cores or central regions that are already in dynamical equilibrium. There are comparatively few examinations of the extended infall regions of clusters due to the lack of wide-field CCD cameras and spectroscopic information. These intermediate density environments contain the structural bridges that lead to the cluster cores. It is expected that because of the longer dynamical timescale they still contain important information about the different stages of the cluster formation and the physical processes that change the morphologies of galaxies. In this regard, it is intriguing to study the galaxy populations that we encounter in the extended areas of nearby clusters.

While high-quality data for distant clusters are continuously being gathered from the Hubble Space Telescope (HST) and large ground-based telescopes, our present knowledge of the

\footnotetext{
5 Author to whom any correspondence should be addressed.
}

systematic properties of galaxies in nearby clusters remains surprisingly limited. Photography-based surveys of clusters in Virgo (Binggeli et al. 1985), Fornax (Ferguson 1989), Centaurus (Jerjen \& Dressler 1997), and Coma (Godwin et al. 1983) are still cited as some of the main references. The significance of these surveys can be understood given the fact that large format, wide-field CCD imagers that enable the mapping of a significant number of nearby clusters became available only recently (e.g., Ferrarese et al. 2012 for Virgo; Pak et al. 2014 for Ursa Major). With the availability of imaging and spectroscopic data from megasurveys such as the Sloan Digital Sky Survey (SDSS), the situation of studies for nearby clusters in the northern hemisphere has been revolutionized. The SDSS data have the advantage of uniform coverage over large areas of sky with photometric and spectroscopic depths that are suitable for studying detailed properties of nearby cluster galaxy populations.

The Virgo cluster is the nearest rich cluster from the Milky Way at a distance of $16.5 \mathrm{Mpc}$ (Jerjen et al. 2004; Mei et al. 2007). The Virgo Cluster Catalog (VCC; Binggeli et al. 1985) has been extensively used and is the primary source catalog for countless studies of properties of cluster galaxies. It contains 2096 galaxies that are distributed within an area of approximately one cluster virial radius $\left(R_{\mathrm{vir}}=6 \mathrm{deg}\right.$ or $1.72 \mathrm{Mpc}$; Hoffman et al. 1980). As the cluster environment already begins to affect the evolution of galaxies located at several virial radii from the center and the Virgo cluster is considered to be a dynamically young cluster (Arnaboldi et al. 2004; Aguerri et al. 2005), investigations of the more extended regions of the Virgo cluster should still contain valuable information about the mass assembly history of the cluster, the physical processes that 


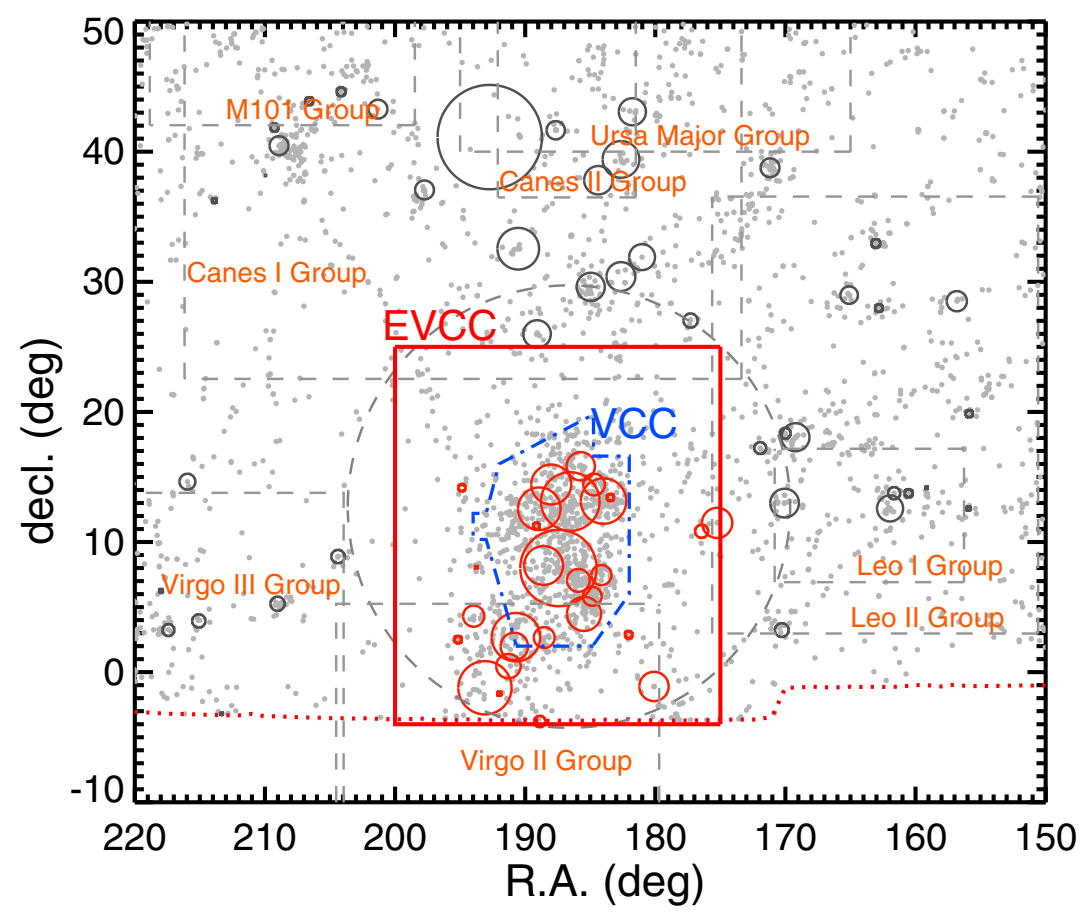

Figure 1. Projected spatial distribution of galaxies in the direction of the Virgo cluster and its surrounding cosmic web. Gray dots are all galaxies from the SDSS DR7 spectroscopic data with radial velocities less than $3000 \mathrm{~km} \mathrm{~s}^{-1}$. The large red rectangle denotes the region of the EVCC, and the region of the VCC is presented by the blue dot-dashed contour. Rectangles with gray dashed lines are outlines of other galaxy groups surrounding the Virgo cluster. The red and gray circles are galaxy groups that are located inside and outside of the EVCC region, respectively, from the galaxy group catalog of Makarov \& Karachentsev (2011). The circle size is proportional to the mean harmonic radius of the group from Makarov \& Karachentsev (2011). For reference, we also present the lower limit of the zero-velocity surface ( $\sim 17$ deg radius, large dashed circle) estimated by Karachentsev \& Nasonova (2010). The red dotted line indicates the coverage limit of the SDSS observations.

(A color version of this figure is available in the online journal.)

govern the morphological transformation of individual galaxies, and the connection of the galaxy distribution with the large-scale filamentary structures around the Virgo cluster (Tully 1982).

In line with the above situation, we present a new catalog of galaxies in the surrounding region of the Virgo cluster, which we call the Extended Virgo Cluster Catalog (EVCC), using publicly available data from the SDSS. The SDSS provides multi-band optical images that are a critical asset for the investigation of galaxy properties. These multi-color images also allow good quality of the morphological classification and in some cases the improvement of the morphological classification of VCC galaxies, which was based on large-scale, blue-sensitive photographic plates with an angular resolution of $10.8 \operatorname{arcsec~mm}^{-1}$. While the membership determination of galaxies in the VCC relied primarily on the visual inspection of the single-band images and galaxy surface brightness, the SDSS can also take advantage of color information and the spectroscopic data for refined classification of galaxies in the Virgo cluster region.

We organize this paper as follows. Section 2 describes the definition of the EVCC region and the selection of galaxies. In Section 3, we introduce two schemes of galaxy classification based on the SDSS imaging and spectroscopic data. Galaxy photometry is described in Section 4 and the final EVCC catalog is presented in Section 5. In Section 6, we present a comparison between the EVCC and VCC. Finally, we summarize our results in Section 7.

\section{SELECTION OF GALAXIES}

\subsection{Data}

The construction of the EVCC is based on the SDSS Data Release 7 (DR7; Abazajian et al. 2009). The SDSS DR7 provides reduced and calibrated images taken in the $u, g, r$, $i$, and $z$ bands with an effective exposure time of $54 \mathrm{~s}$ in each band (see also Stoughton et al. 2002). The pixel scale of 0.396 arcsec and the average seeing of 1.4 arcsec correspond to a physical size of $32 \mathrm{pc}$ and $112 \mathrm{pc}$, respectively, at a Virgo cluster distance of $16.5 \mathrm{Mpc}$ (i.e., a distance modulus $m-M=31.1$; Jerjen et al. 2004; Mei et al. 2007). The SDSS spectroscopic survey nominally covers all galaxies brighter than $r \leqslant 17.77$ and $r$-band Petrosian half-light surface brightnesses $\mu_{50} \leqslant 24.5 \mathrm{mag} \operatorname{arcsec}^{-2}$ (Strauss et al. 2002). It provides fiber spectra with a wavelength coverage between $3800-9200 \AA$ at a resolution of $R=2000$. The fiber has an angular diameter of $3 \mathrm{arcsec}$ that corresponds to $240 \mathrm{pc}$ at a distance of Virgo, providing spectral information for the very central region of the galaxies. We took advantage of the SDSS spectroscopic data for the determination of galaxy membership and classification of spectroscopic morphology (see Sections 2.4 and 3.2). The SDSS imaging data allow detailed morphological classification and accurate photometry of galaxies (see Sections 3.1 and 4 for details).

\subsection{Region of the EVCC}

The SDSS spectroscopic data were searched in the window of $175 \mathrm{deg}<$ R.A. (J2000) $<200 \mathrm{deg}$ and $-4 \mathrm{deg}<$ decl. (J2000) $<$ $25 \mathrm{deg}$, which defines the nominal area of the EVCC (large red rectangle in Figure 1). This region extends to the lower limit of the zero-velocity surface of the Virgo cluster, about $17 \mathrm{deg}$ radius from the cluster center, estimated by Karachentsev \& Nasonova (2010), which separates the galaxies in a given galaxy cluster against the global cosmic expansion. In contrast, the VCC region covers a smaller area within a radius of $\sim 10$ deg (blue dot-dashed contour in Figure 1). The EVCC 


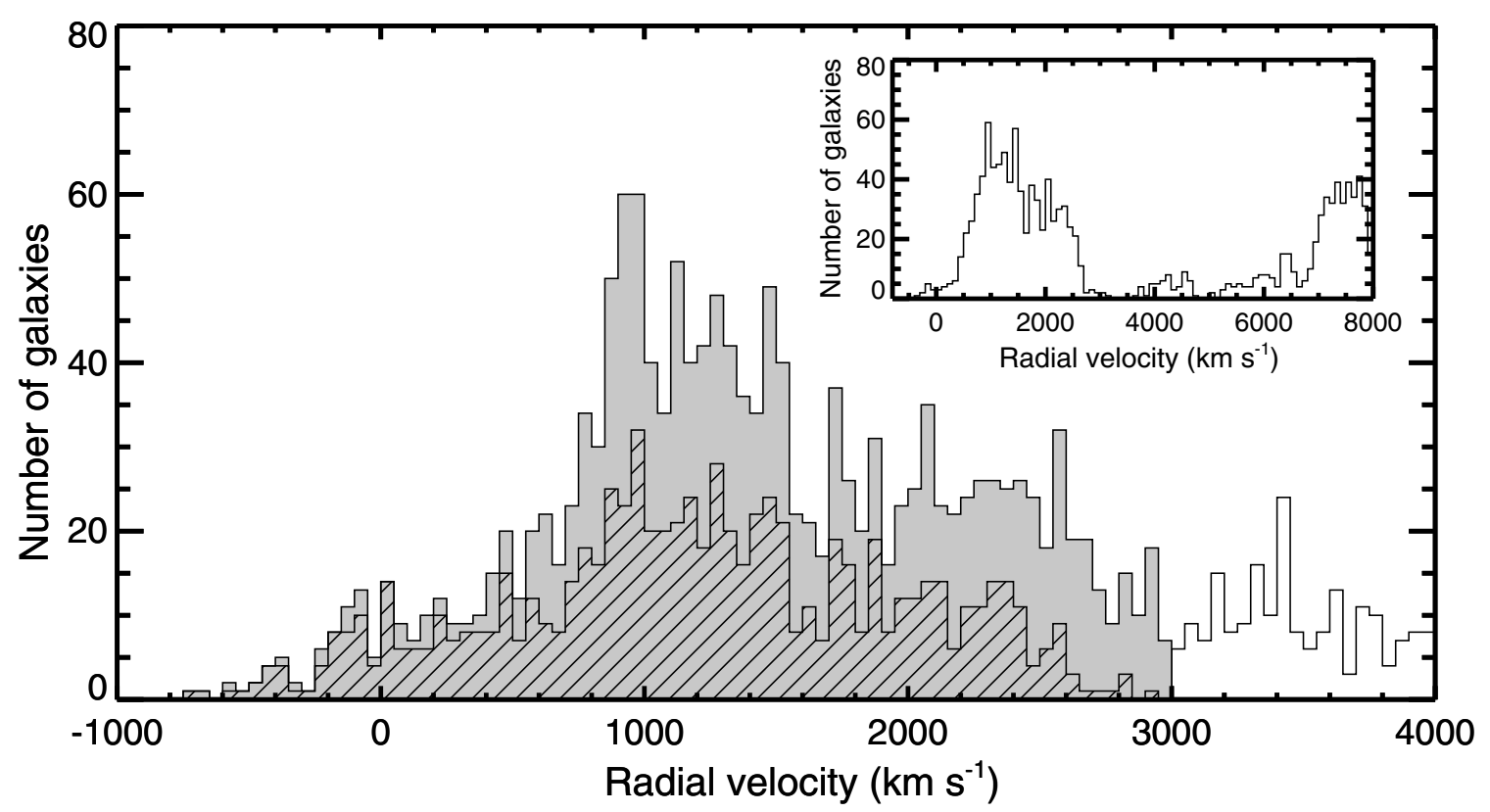

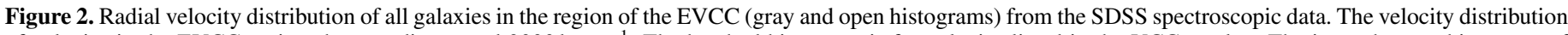

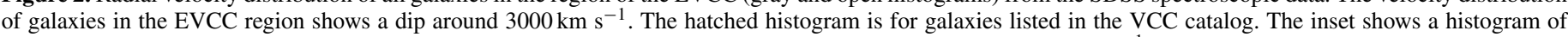

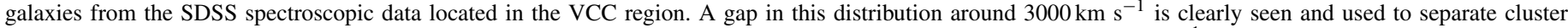
members from background. The selected galaxies included in the EVCC are those with radial velocities less than $3000 \mathrm{~km} \mathrm{~s}^{-1}$ (gray histogram).

area $\left(725 \mathrm{deg}^{2}\right)$ is 5.2 times larger than the footprint of the VCC $\left(140 \mathrm{deg}^{2}\right)$, reaching up to 3.5 times the virial radius of the Virgo cluster.

The EVCC region generally avoids overlap with other distinct galaxy groups in the vicinity (gray dashed line rectangles in Figure 1, Fouque et al. 1992; Garcia 1993; Giuricin et al. 2000). From the nearby galaxy group catalog of Makarov \& Karachentsev (2011), the EVCC region includes a complex of subgroups around the main body of the Virgo cluster (red circles in Figure 1) and the neighboring galaxy groups are also located outside of the EVCC region (gray circles in Figure 1). The southern extension of the Virgo cluster at decl. $<5 \mathrm{deg}$, which is a part of the Local Supercluster (de Vaucouleurs \& de Vaucouleurs 1973; Tully 1982; Sandage et al. 1985a; Hoffman et al. 1995), is well covered by the EVCC, while the VCC region included only the most northern tip of this structure. However, the EVCC does not cover the area below decl. $=-4$ deg where the Virgo II Group (de Vaucouleurs 1961; de Vaucouleurs \& de Vaucouleurs 1973) is located, owing to the coverage limit of the SDSS observations (see the red dotted line in Figure 1).

\subsection{Selection of Sample Galaxies}

We initially focus on galaxies with radial velocities that are available in the SDSS spectroscopic data since the velocity is a suitable information for cluster membership of galaxies in a galaxy cluster without significant foreground and background. In Figure 2, we present the velocity distribution of galaxies in the region of the EVCC (gray and open histograms). We selected 1324 galaxies with radial velocities less than $3000 \mathrm{~km} \mathrm{~s}^{-1}$ in the EVCC region (i.e., gray histogram). The value of $3000 \mathrm{~km} \mathrm{~s}^{-1}$ was adopted as an upper limit in the velocity distribution of the Virgo galaxies since a well-defined gap around $3000 \mathrm{~km} \mathrm{~s}^{-1}$ has been acknowledged by Binggeli et al. (1993; see the inset of Figure 2), while galaxies in the EVCC region exhibit a dip in their radial velocity distribution owing to the contribution of galaxies outside of the VCC region.
While SDSS spectroscopic observations generally cover galaxies down to an apparent magnitude of $r=17.77$, bright, bulge-dominated galaxies with $r<14.5$ were rejected from the SDSS spectroscopic target list as they cause saturation problems (Blanton et al. 2005a, 2005b). Another source of incompleteness are fiber conflicts, where neighboring fibers could not be placed closer than 55 arcsec (McIntosh et al. 2008). To complete the EVCC catalog with galaxies for which radial velocities were not measured in the SDSS, we extracted independent spectroscopic data from the NASA/IPAC Extragalactic Database (NED) for 265 galaxies that are located in the EVCC region. These galaxies cover a wide range in magnitude, $8<r<19$. As a result, the EVCC contains a total of 1589 galaxies, 676 of which are included only in the EVCC (see also Figure 28 and Section 6.4).

To test the consistency between SDSS and NED, we examined the radial velocities of 498 EVCC galaxies which are available in the SDSS and NED. The mean difference $\left(2.6 \mathrm{~km} \mathrm{~s}^{-1}\right)$ and standard deviation $\left(105 \mathrm{~km} \mathrm{~s}^{-1}\right)$ of radial velocity turn out negligible. Furthermore, we found that there is no significant deviation of the radial velocity depending on the magnitude range. Specifically, faint galaxies $(r>16)$ also show similar residuals (i.e., $10 \pm 103 \mathrm{~km} \mathrm{~s}^{-1}$ ). Consequently, we conclude that radial velocity information of our sample is not significantly hampered by difference of compiled sources.

The spectra of most SDSS galaxies are obtained from fibers positioned on or close to the galactic center, but some galaxies in the SDSS have their spectrum taken at an off-center position. Some galaxies have even multiple spectra observed at various off-center positions. In the latter case, we select the fiber spectrum obtained at the location closest to the center to minimize the uncertainties of radial velocities due to rotation or peculiar motion within a galaxy. About $10 \%$ of the SDSS data included in the EVCC have off-center spectra.

We also examined the reliability of radial velocities obtained from the off-center spectra. We select galaxies whose spectra from the SDSS are off center by more than 5 arcsec from the photometric galaxy center and compare their radial velocities 

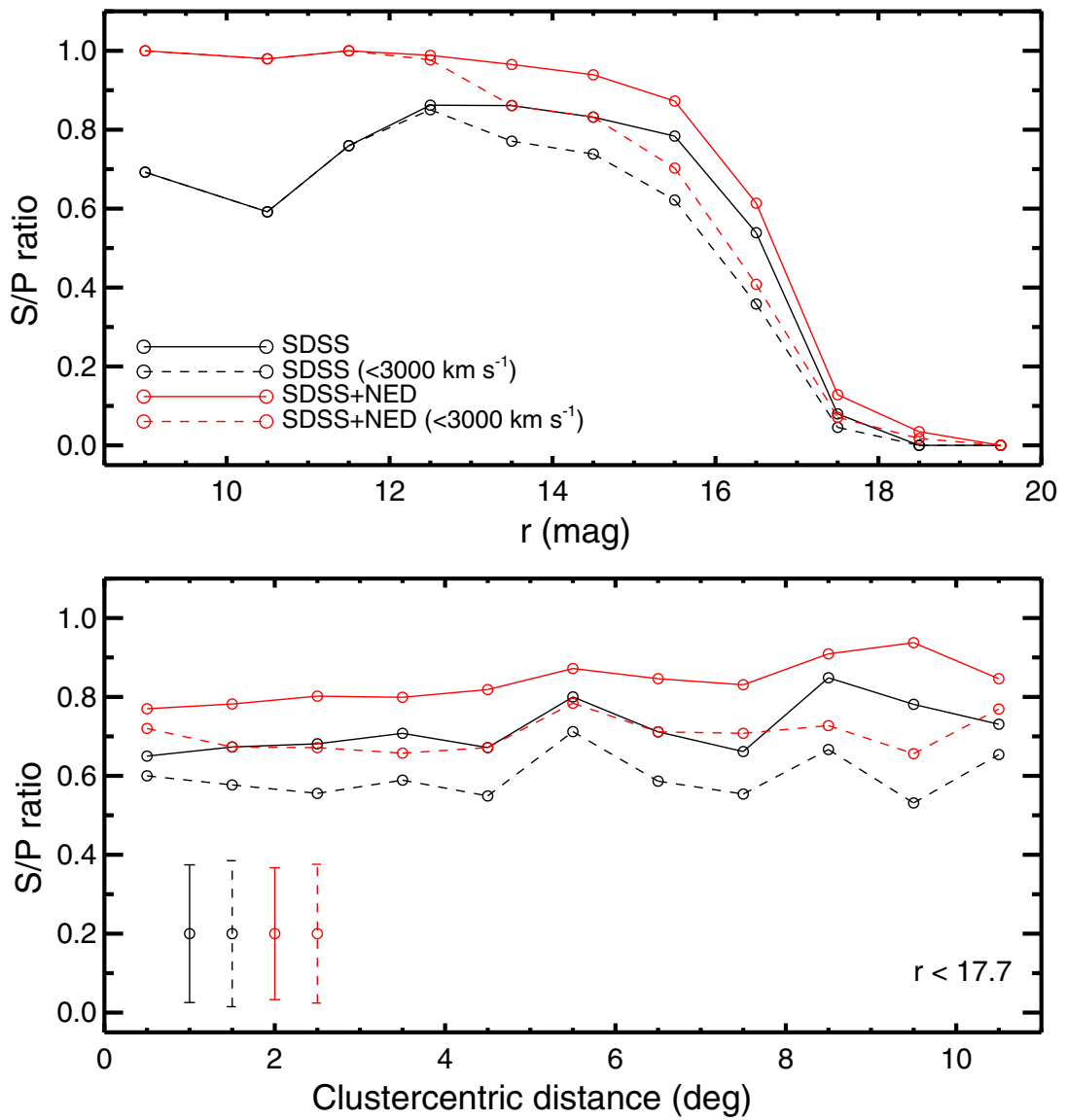

Figure 3. Top: $\mathrm{S} / \mathrm{P}$ ratio (the fraction of certain and possible $\mathrm{VCC}$ member galaxies with available spectroscopic data) against the $r$-band magnitude. Bottom: the $\mathrm{S} / \mathrm{P}$ ratio of galaxies brighter than $r=17.7$ with respect to the clustercentric distance from the Virgo cluster center. The red and black lines indicate $\mathrm{S} / \mathrm{P}$ ratios obtained from the SDSS data with and without the NED data, respectively. Dashed and solid lines indicate galaxies with $c z<3000 \mathrm{~km} \mathrm{~s}^{-1}$ and with all redshift data, respectively. Error bars in the bottom panel denote the mean values of Poisson error in different $\mathrm{S} / \mathrm{P}$ ratios.

(A color version of this figure is available in the online journal.)

with independent measurements in NED where spectra are observed on their center. We found that the radial velocities of these galaxies show no significant difference with those from the NED (i.e., $12 \pm 98 \mathrm{~km} \mathrm{~s}^{-1}$ ), indicating no appreciable systematic bias with respect to radial velocities from on-center spectra.

In Figure 3, we estimate the number ratio of VCC certain and possible member galaxies with available radial velocities from SDSS and NED to those with SDSS photometric data (hereafter $\mathrm{S} / \mathrm{P}$ ratio) as a function of magnitude (top panel) and clustercentric distance (bottom panel). The $\mathrm{S} / \mathrm{P}$ ratio is obtained for the SDSS data with (red solid line) and without (black solid line) the NED data. The S/P ratio of galaxies with $c z<$ $3000 \mathrm{~km} \mathrm{~s}^{-1}$ (i.e., $\mathrm{S} / \mathrm{P}_{3000}$ ratio, dashed lines) is also considered. The overall feature of the $\mathrm{S} / \mathrm{P}_{3000}$ ratio versus the magnitude is that it drops to a 50\% completeness level around $r \sim 16.5$ $\left(M_{r}=-14.6\right)$. Galaxies fainter than $r=18\left(M_{r}=-13.1\right)$ remain largely undetected by the SDSS and NED data. In the case of the S/P ratio for only the SDSS data, a prominent paucity of spectroscopic data for bright galaxies with $r<13$ is seen. This highlights the saturation problem of SDSS spectroscopic observations mentioned by Blanton et al. (2005a, 2005b).

However, by adding the NED data, the S/P ratio increases to $\sim 100 \%$ for about $r<14$. At a given magnitude, the $\mathrm{S} / \mathrm{P}_{3000}$ ratio is lower than the $\mathrm{S} / \mathrm{P}$ ratio in the magnitude range of $13<r<18$. This indicates that some fainter galaxies in the morphology-based VCC are absent in the EVCC because their redshifts are larger than $3000 \mathrm{~km} \mathrm{~s}^{-1}$ (see also Section 6.4).
In addition, we examine the $\mathrm{S} / \mathrm{P}$ ratio for galaxies brighter than the magnitude limit of SDSS spectroscopic observations (i.e., $r=17.7$ ) with respect to the clustercentric distance from the Virgo (see the bottom panel of Figure 3). The S/P ratio shows no dependence on the location within the cluster, considering Poisson error $(\sim \pm 0.18)$ in the $\mathrm{S} / \mathrm{P}$ ratio (see the error bars in the bottom panel of Figure 3). This suggests that the average angular separation between galaxies at the distance of the Virgo cluster is large enough to avoid the problem of fiber collisions in the SDSS observations (i.e., 55 arcsec; Strauss et al. 2002).

\subsection{Membership}

Cluster membership determination of galaxies in the VCC was essentially based on optical morphology as follows (for further details see Sandage \& Binggeli 1984; Binggeli et al. 1985 for details). Cluster member early-type dwarfs and irregular galaxies are well distinguished from the background galaxies, since they follow a well-established correlation between magnitude and surface brightness (Binggeli \& Cameron 1991; Boselli et al. 2008). Thus, at a given magnitude, these cluster members exhibit lower surface brightness than the majority of background counterparts. In the case of spiral galaxies, luminosity classes combined with apparent galaxy size were used to distinguish members from background objects (van den Bergh 1960). Additionally, resolution into star-forming knots provided a complementary criterion for late-type galaxies; i.e., member 


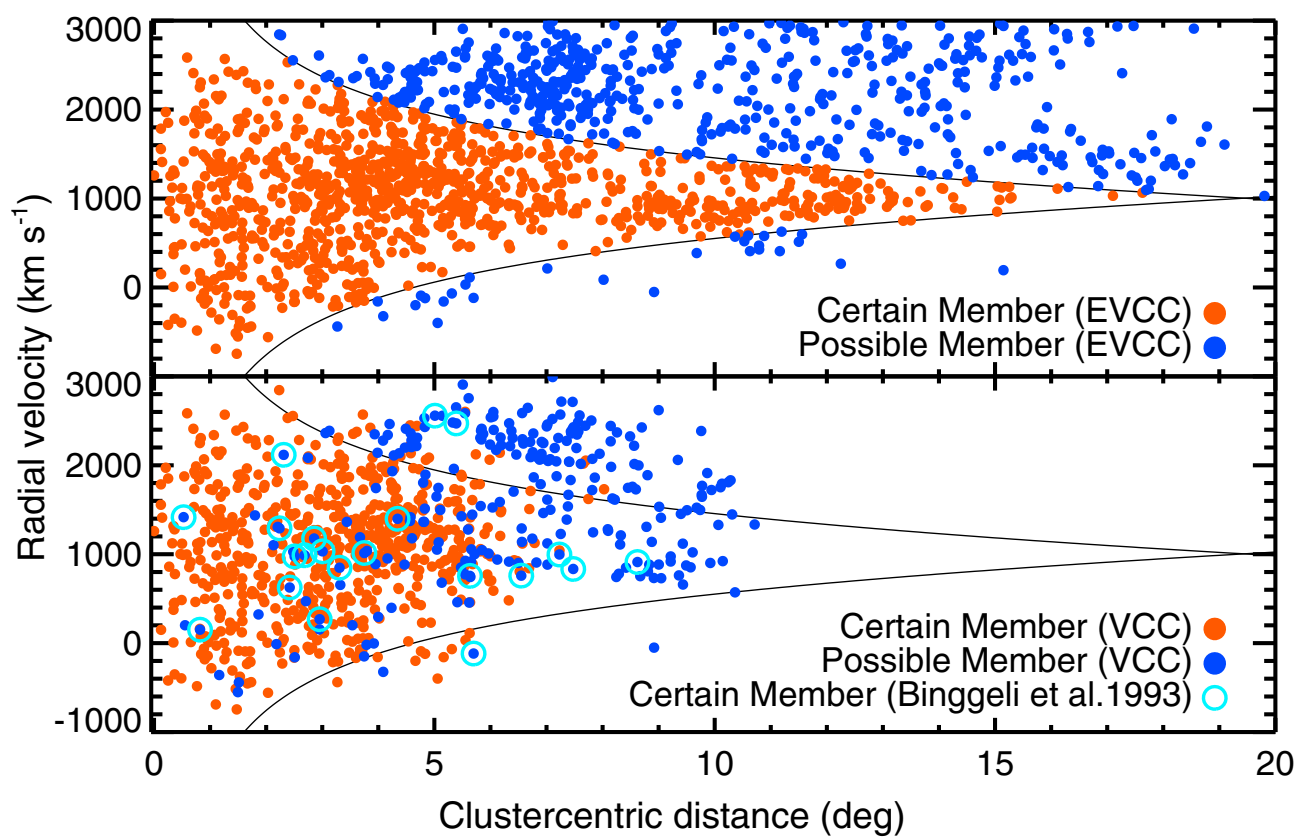

Figure 4. Top: radial velocities of EVCC galaxies against the projected radius from the cluster center. The solid lines indicate the model caustic curves using the infall model of Praton \& Schneider (1994). Red and blue dots are member and possible member galaxies defined as galaxies located inside and outside of the model lines, respectively. Bottom: radial velocities of VCC galaxies against the projected radius from the cluster center. Membership is taken from the VCC and radial velocity information is from the SDSS or NED. Cyan open circles are possible member galaxies in the VCC which were re-classified as certain members based on the radial velocity information from Binggeli et al. (1993).

(A color version of this figure is available in the online journal.)

Table 1

Primary Morphology

\begin{tabular}{lcl}
\hline \hline Type & Digital Code & \multicolumn{1}{c}{ Subclass } \\
\hline Elliptical & 100 & \\
Disk galaxy & $2[0,1][0-7]$ & [no-bar $=0 /$ bar $=1]$ [subtype: $0=0, \mathrm{a}=1, \mathrm{~b}=2, \mathrm{c}=3, \mathrm{~d}=4, \mathrm{~m}=5, \mathrm{~S}=6$, edge-on $=7]$ \\
Irregular & $3[0,1] 0$ & [number of prominent $\mathrm{H}$ II regions: none to low $=0$, high $=1]$ \\
Early-type dwarf galaxy & $4[0,1][0,1]$ & [subtype: $\mathrm{dE}=0, \mathrm{dS} 0=1]$ [no nucleus $=0 /$ nucleus $=1]$ \\
\hline
\end{tabular}

galaxies exhibit well-resolved $\mathrm{H}$ II regions and stellar associations compared to the background counterparts. Binggeli et al. (1993) subsequently demonstrated with newly available radial velocity data that morphology is a powerful method to separate cluster members and background galaxies with a 95\% success rate. These authors noted that in the case of the Virgo cluster membership status can be best determined with radial velocity data because of a distinct gap in the velocity distribution behind the Virgo cluster at $3000 \mathrm{~km} \mathrm{~s}^{-1}$.

We divide the EVCC galaxies into two subsamples using a spherical symmetric infall model that defines the limits of the infall velocity as a function of distance from the cluster center (Praton \& Schneider 1994). Under the assumption that galaxy clusters are small density perturbations in the early universe, a spherical infall model predicts the galaxy motions based on the spherical accretion onto a point mass in a uniform and expanding universe. The density perturbations eventually have broken away from the general Hubble expansion as a result of the dominating gravitational force out to a maximum distance (i.e., turnaround radius), allowing material to move inward. The infall motion produces a caustic curve in a galaxy cluster which consists of all galaxies whose infall motion is larger than the local Hubble flow. The spherical infall model can be calculated from the virial radius and the line-of-sight velocity dispersion of a cluster (Praton \& Schneider 1994; Abdullah et al. 2011). Consequently, a caustic curve for a model of the Virgo cluster is obtained using $\Omega_{0}=0.3$ (Jing et al. 1995), a velocity dispersion $\sigma_{v}=800 \mathrm{~km} \mathrm{~s}^{-1}$ (Binggeli et al. 1993), and a virial radius of $1.8 \mathrm{Mpc}$ (Hoffman et al. 1980).

In Figure 4 (top panel), we plot the radial velocities of the EVCC galaxies against the Virgocentric distance, measured from M87, along with the model caustic curves. We define certain member (red dots) and possible member galaxies (blue dots) as those located inside and outside of the model lines, respectively. For comparison, we also present VCC galaxies in Figure 4 (bottom panel) using membership classification from the VCC. Most VCC galaxies classified as certain member (red dots) based on the morphology are inside of the model lines, confirming they are true members in terms of their radial velocity information. It is worth noting that some possible member (blue dots) galaxies classified in the VCC are also enveloped in the caustic curves showing relatively small deviation of their radial velocities to the cluster's systemic velocity. Binggeli et al. (1993) investigated the membership of 144 galaxies in the VCC using available radial velocity information at that time. As a result, about $22 \%$ (21 of 94 ) of possible member galaxies were reclassified as certain member galaxies (see Table 1 of Binggeli et al. 1993). Note that all but three of these galaxies are located inside of the caustic curves (cyan circles in the bottom panel of Figure 4).

By definition, galaxies that are located outside of the caustic curves of the infall model should be considered as non-member 
galaxies of the Virgo cluster. Nevertheless, the projected spatial distribution of these galaxies appears to be similar to that of possible member galaxies in the VCC (see Figure 21 of Section 6.2). Furthermore, in the VCC, substructures of the Virgo cluster, including the $\mathrm{W}, \mathrm{W}^{\prime}$, and $\mathrm{M}$ clouds and southern extension region, are dominated by possible member galaxies (Binggeli et al. 1985, 1987, 1993; Sandage et al. 1985a), and some results suggest that they are connected with the M87 and M49 clusters (see Yoon et al. 2012 and references therein). Therefore, we characterize galaxies located outside of the infall model lines as possible members, following the VCC terminology.

\section{MORPHOLOGY CLASSIFICATION}

The SDSS data allow us to investigate the relationship between photometric and spectroscopic properties of galaxies. We introduce two classification schemes using the SDSS imaging and spectroscopic data. In addition to the traditional morphological classification by visual inspection of images ("primary morphology"), we also classify galaxies based on their spectroscopic features ("secondary morphology"). The primary morphological classification follows the scheme of the morphological classification used in the VCC. The secondary morphological classification relies on the spectral energy distribution (SED) shape and presence of $\mathrm{H} \alpha$ emission/absorption lines. The morphological classification for all galaxies was independently performed by three members of our team (H.J., T.L., and E.C.S.).

\subsection{Primary Morphology}

The morphological classification was carried out by carefully examining both monochromatic $g-, r$-, and $i$-band images and gri combined color images of the SDSS data. Some of the advantages of using digital images over photographic plates is the ability to zoom in, vary contrast levels, as well as cut levels to investigate low and high surface brightness features in detail. The EVCC galaxies were subdivided into 21 classes on the basis of the extended Hubble morphological classification scheme. These classes are summarized in Table 1 and illustrated in Figure 5. We briefly describe the most important features here and refer to the following main references for a comprehensive discussion and illustration of the different types: Sandage \& Binggeli (1984), Sandage et al. (1985b), Binggeli \& Cameron (1991), Sandage \& Bedke (1994). ${ }^{6}$

1. Elliptical galaxy $(E)$. Elliptical galaxies show high surface brightness spheroidal shape and a featureless envelope.

2. Lenticular galaxy (SO). The main feature that discriminates between an E and an S0 galaxy is the presence of a disk. S0 galaxies have a smooth bulge and disk component with a distinct change in surface brightness at the transition radius when seen face-on. Edge-on S0s display a lens shape.

3. Spiral $(S a-S d)$. The classification criteria for spiral galaxies are the relative strength of the bulge and the degree of tightness of the spiral arms defined by bright H II regions. Qualitatively, early-type spirals (e.g., $\mathrm{Sa}$ and $\mathrm{Sb}$ ) have a large bulge and tightly wrapped spiral arms, while latetype spirals (e.g., Sc and Sd) have a small bulge and patchy open spiral arms.

\footnotetext{
6 http://ned.ipac.caltech.edu/level5/Shapley_Ames/frames.html
}

4. Early-type dwarf galaxy ( $d E$ and $d S O)$. A low surface brightness galaxy characterized by elliptical isophotes and a smooth surface brightness profile. Overall colors are generally red, but some faint dEs show blue colors. Among the $\mathrm{dE}$ class, some galaxies show a distinct bulge-disk transition and/or asymmetric features (e.g., EVCC 976 in Figure 5). They are classified as dS0. If an unresolved, starlike nucleus at the center is present in a $\mathrm{dE}$ or a dS0, they are called $\mathrm{dE}(\mathrm{N})$ or $\mathrm{dS} 0(\mathrm{~N})$.

5. Sm. Sm galaxies are called the Magellanic type spirals and are the latest type where traces of spiral patterns are still visible.

6. Barred galaxy. Barred galaxies exhibit a large-scale linear bar feature crossing the nucleus.

7. High surface brightness irregular (HSB Irr) and low surface brightness irregular (LSB Irr). Irregular galaxies have a chaotic morphology. We use the surface brightness to separate the blue compact dwarf (BCD) type from the "Im" galaxies similar to the luminosity class in the Revised Shapley-Ames Catalog (Sandage \& Tammann 1981). The EVCC irregulars of highest surface brightness are called HSB Irr. Their appearance is dominated by a single or sometimes group of bright, centrally located $\mathrm{H}$ II regions. We classified all irregular EVCC galaxies as if they were at the distance of the Virgo cluster (assuming an appropriate line-of-sight depth). However, HSB galaxies are the most difficult to correctly determine using only redshift and angular size. It is possible that there are a few background spiral galaxies in the HSB class. The LSB Irr galaxies are irregular galaxies with low surface brightness similar to dEs but with irregular isophotes. Based on the $r$-band peak surface brightness values and half-light radius (R50) returned from SExtractor, at all given magnitudes, HSB Irr galaxies are found to have systematically higher central surface brightness (difference of about 1 mag $\operatorname{arcsec}^{-2}$ ) and smaller angular size (a factor of about 2 in R50) compared to the LSB Irr counterparts.

8. Edge-on galaxy. Edge-on galaxies are spiral galaxies seen edge-on and thus did not allow a more detailed morphological classification within the "disk galaxy" class.

9. $S$. Galaxies that have relatively small angular size ${ }^{7}$ and did not have unique features within the spiral galaxy classifications scheme.

\subsection{Secondary Morphology}

The shape of the SED is related to the color of a galaxy while the stellar absorption and emission lines trace star formation activity within a galaxy (Kennicutt 1992). In this context, we subdivide all galaxies into red- and blue-type galaxies based on the overall shape of their SEDs. We construct a model SED with an age of $1 \mathrm{Gyr}$ and solar metallicity from the population model (Bruzual \& Charlot 2003; see the red solid curves in Figure 6) and compare it with the observed SED for each galaxy. When the wavelength of the peak intensity in the SED is shorter or longer compared to that of the model, we classify the galaxies as blue or red, respectively. We further subdivide the blue and red galaxies into two subclasses based on the presence of Balmer absorption and emission lines. Consequently, all galaxies included in the EVCC are classified as four types of secondary morphologies as follow (see Figure 6 for examples).

\footnotetext{
7 The majority (about $87 \%$ ) of S-type galaxies have a Kron radius smaller than 50 arcsec, while only a small fraction (about 11\%) of normal spiral galaxies $(\mathrm{Sa} / \mathrm{Sb} / \mathrm{Sc} / \mathrm{Sd})$ belong to these small objects.
} 

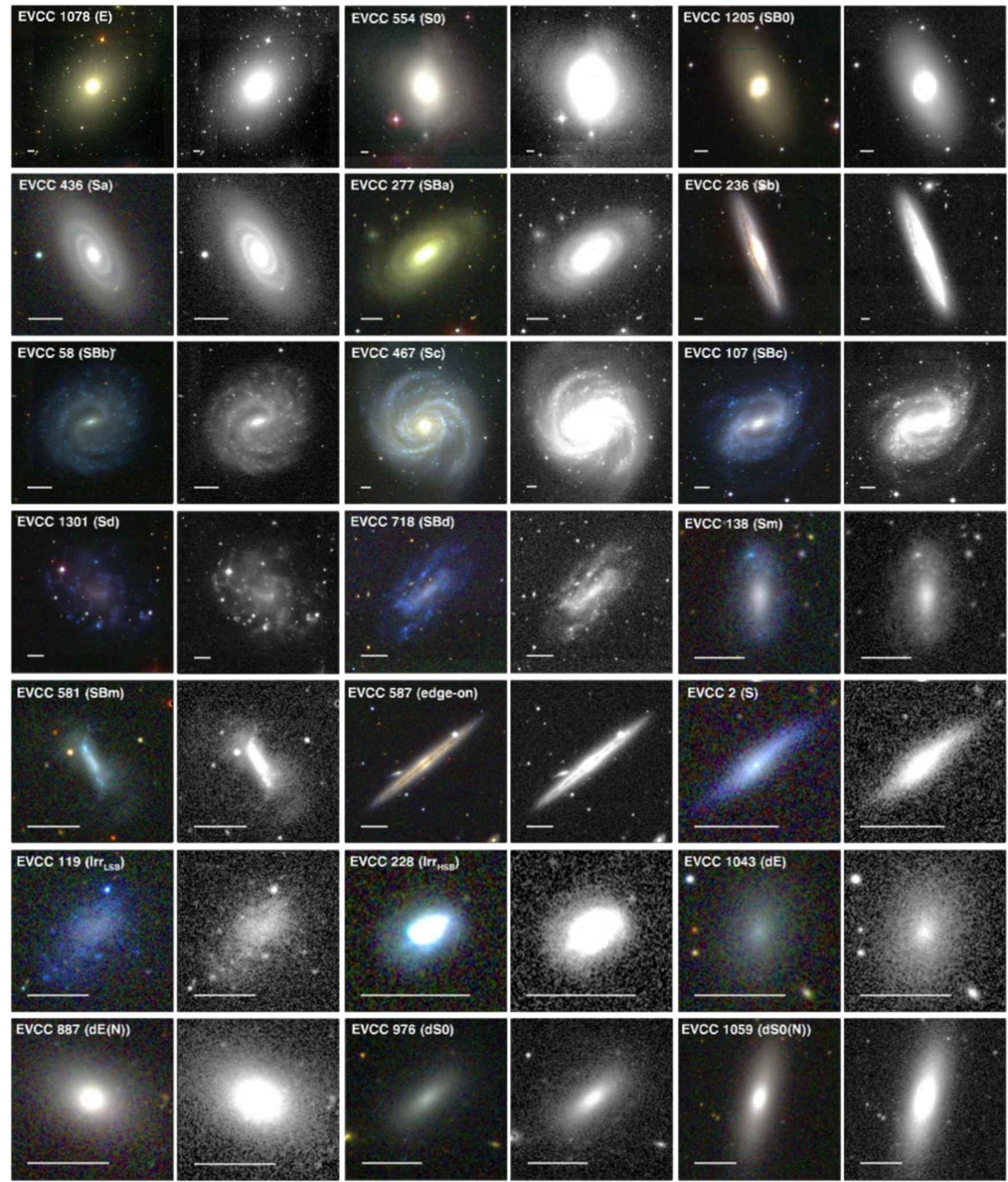

Figure 5. Examples of galaxies with various primary morphologies. For each galaxy we show the gri combined color image (left) and the $r$-band grayscale image (right). In each panel, the solid bar corresponds to $30 \mathrm{arcsec}(2.4 \mathrm{kpc})$ on the sky. North is up and east is to the left. The EVCC number and the morphological type are also given in the upper left corner of the color image.

(A color version of this figure is available in the online journal.) 

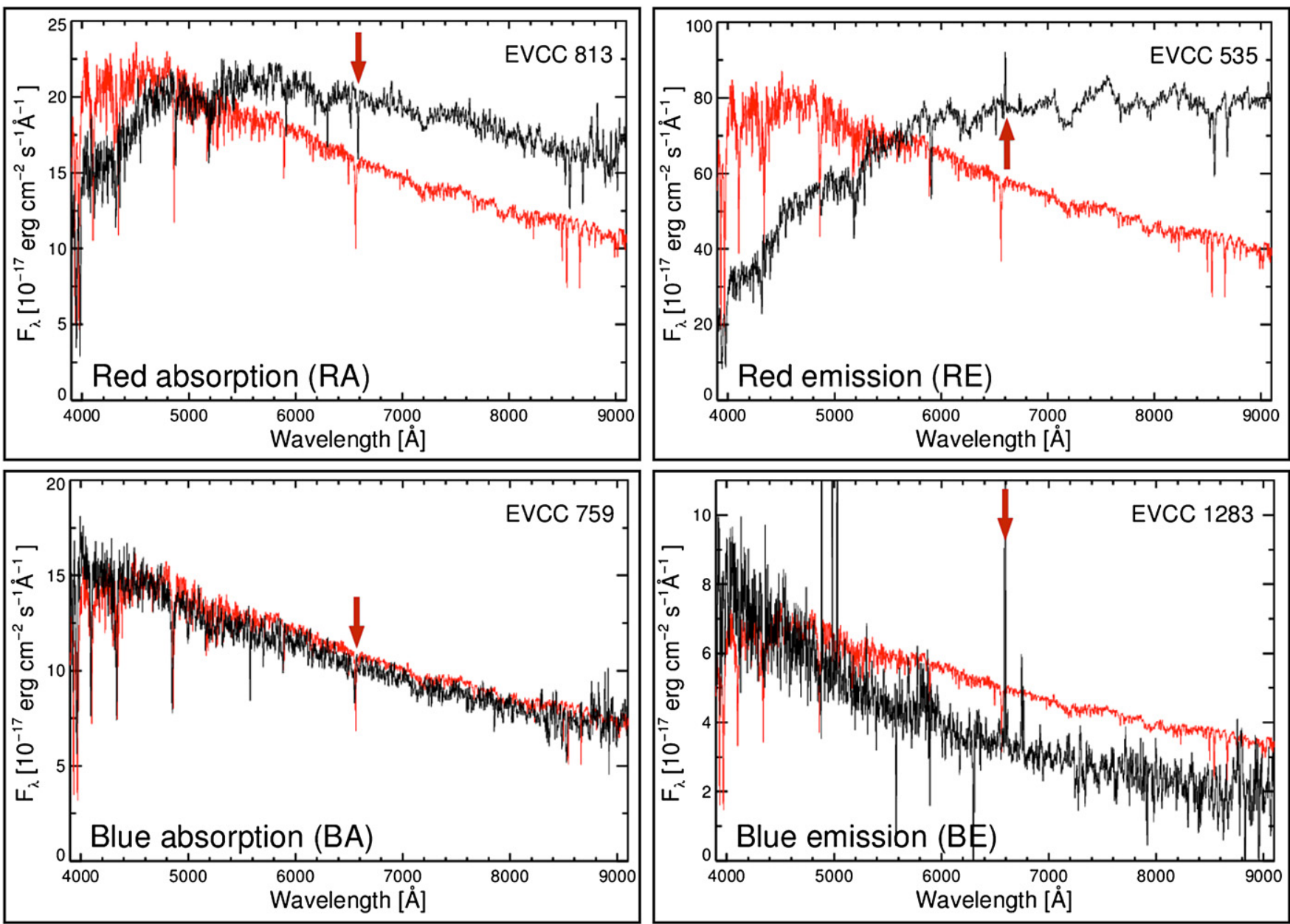

Figure 6. Examples of galaxies with various secondary morphologies. Red arrows indicate the H $\alpha$ line in emission or absorption. In each panel, a model SED with an age of $1 \mathrm{Gyr}$ and solar metallicity from the population model is overplotted as a red solid curve. Secondary morphology is classified from comparing this model SED with the observed spectrum of each galaxy (see the main text for details).

(A color version of this figure is available in the online journal.)

1. Red absorption (RA) galaxy. overall SED shape of a typical early-type red galaxy with an $\mathrm{H} \alpha$ absorption line.

2. Red emission (RE) galaxy. overall SED shape of a typical early-type red galaxy, but it has $\mathrm{H} \alpha$ in emission.

3. Blue absorption (BA) galaxy. overall SED shape of a typical blue galaxy, and has an $\mathrm{H} \alpha$ absorption line.

4. Blue emission (BE) galaxy. overall SED shape of a typical blue galaxy with an $\mathrm{H} \alpha$ emission line. In many cases, many emission lines (e.g., $\mathrm{H} \alpha, \mathrm{H} \beta$, and [O III] 25007 ) are prominent compared to the continuum.

For galaxies with multiple SDSS spectra taken at various locations, we used the fiber spectrum obtained closest to the photometric center of the galaxy (see also Section 2.3). We note that our secondary morphology generally provides spectral information of the central region of a galaxy due to the 3 arcsec (i.e., $240 \mathrm{pc}$ ) fiber aperture of the SDSS spectrograph. Our secondary morphology classification is somewhat coarse, because galaxies belonging to the same secondary morphology can have a different SED slope and $\mathrm{H} \alpha$ strength.

\subsection{Comparison between Primary and Secondary Morphology}

It has been known that the Hubble type of a galaxy closely correlates with its spectrum (Sánchez Almeida et al. 2010 and references therein). In Figure 7, we present a two-dimensional histogram comparing the two morphological classifications (primary versus secondary) for the 1189 EVCC galaxies. We excluded 135 galaxies in the EVCC whose spectra are off center by more than 5 arcsec from the photometric galaxy center. Figure 7 also presents the morphology distribution (filled histogram) as well as the percentage of early-type (i.e., $\mathrm{E} / \mathrm{dE}$ and S0/dS0; hatched histogram of upper panel) and red (RA and RE; hatched histogram in the right panel) galaxies in each morphology bin. We summarize the main features of our comparison between primary and secondary morphology.

1. We find an expected general trend between photometric (i.e., primary) and spectroscopic (i.e., secondary) morphologies. Most (78\%) early-type galaxies exhibit red spectra (i.e., RA type) and the majority (73\%) of late-type galaxies show blue spectra (i.e., BE type). While our secondary morphology is obtained from the spectrum of the central region of the galaxy, it signifies that the secondary morphology also reflects global characteristics of the galaxies. However, the correlation between these two morphologies exhibits some scatter for both morphologies in the sense that there are numerous outliers in the main trend along the diagonal.

2. The majority (95\%) of galaxies with red absorption line spectra are early-type galaxies (E/dE and S0/dS0). Only 


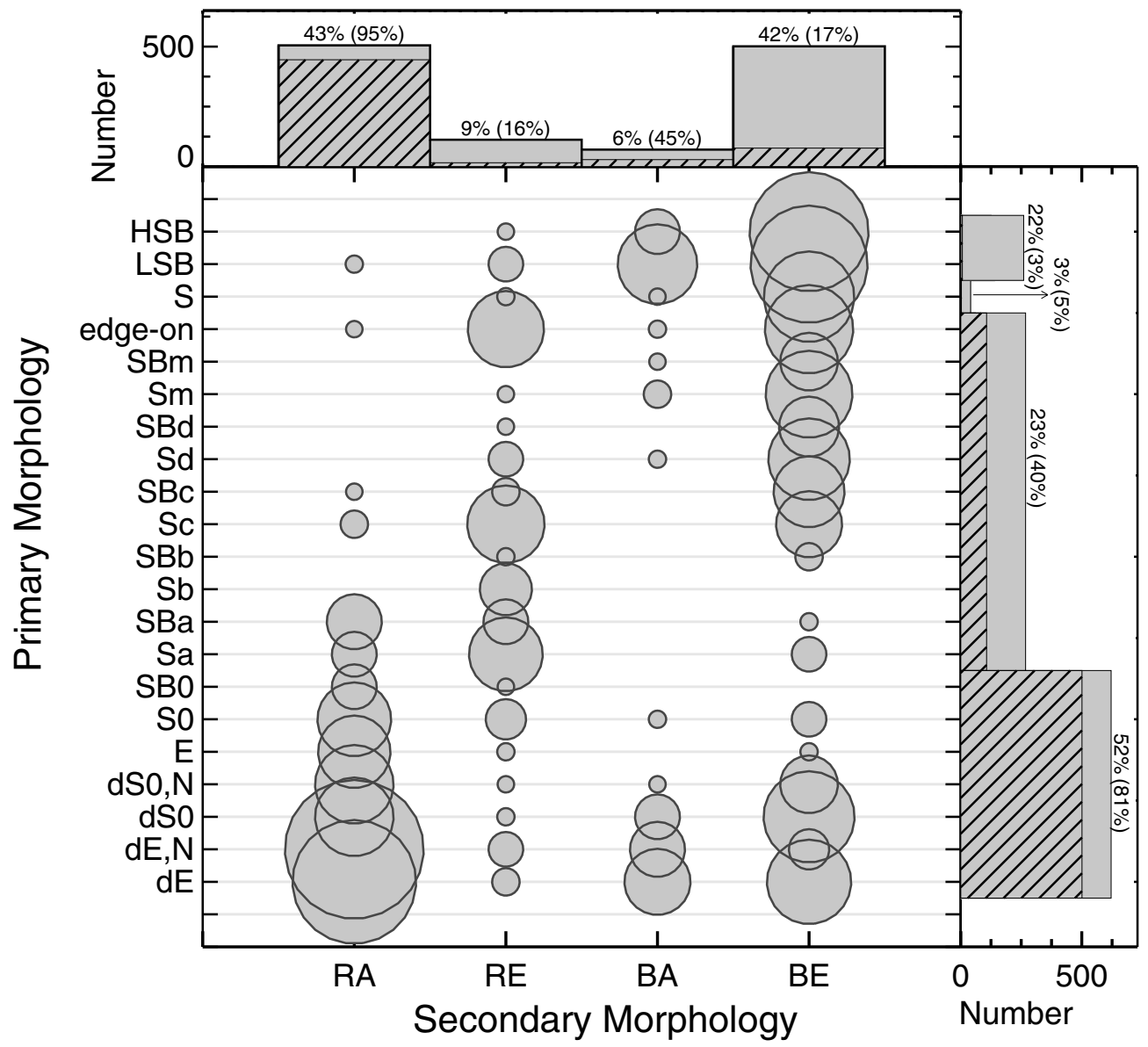

Figure 7. Comparison between the primary and secondary morphology of 1189 galaxies in the EVCC. The symbol size is proportional to the number of galaxies. In the upper and right panels, the gray filled histogram indicates the fraction of galaxies included in the secondary (RA, RE, BA, and BE) and primary morphology (early-type, spiral, and Irr), respectively. The hatched histograms represent the fraction of early-type galaxies (i.e., E/dE and S0/dS0) for each secondary morphology (upper panel) and that of red galaxies (i.e., RA and RE) for each primary morphology (right panel). The numbers above the histogram correspond to the fraction of morphology. The numbers in parentheses are the fraction of early-type and red (RA/RE) galaxies in each morphology bin for the secondary and primary classification, respectively.

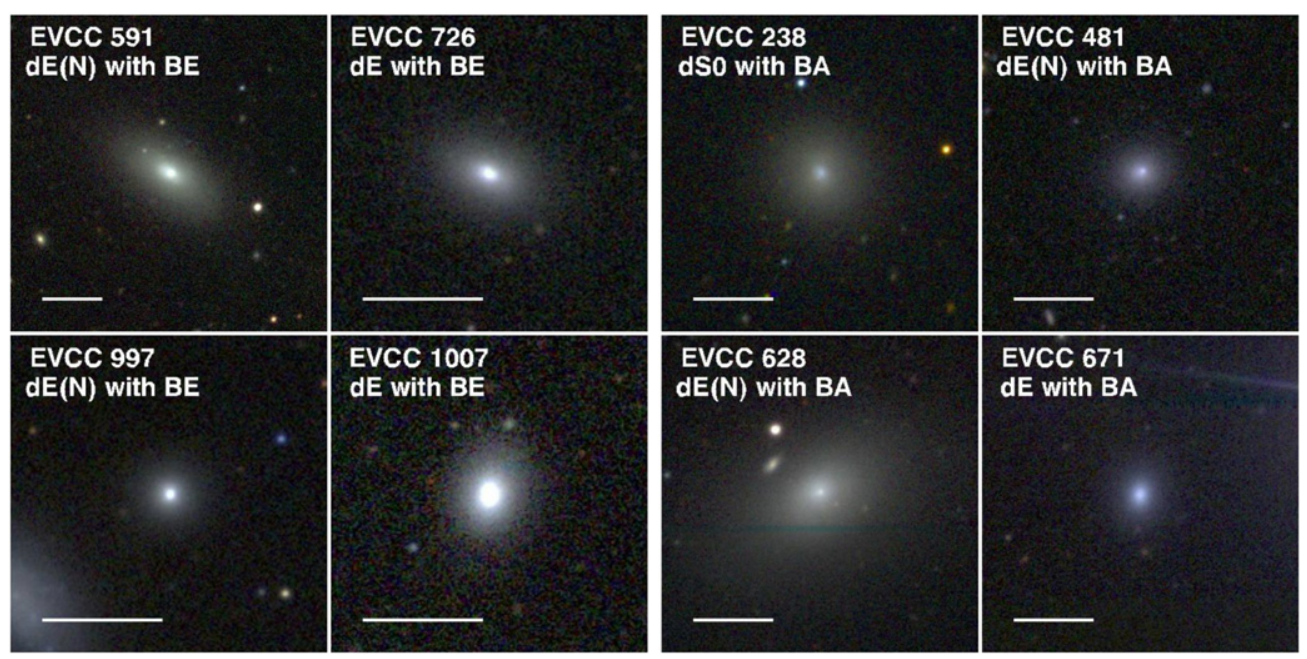

Figure 8. Examples of BE- (left panels) and BA-type (right panels) early-type dwarf galaxies that exhibit a blue core at their centers. The solid bar corresponds to 30 arcsec on the sky. North is up and east is to the left.

(A color version of this figure is available in the online journal.)

a small fraction (3\%) of RA-type galaxies are bulgedominated $\mathrm{Sa} / \mathrm{SBa}$ galaxies. There is a lack $(2 \%)$ of latetype galaxies (Irrs and spiral galaxies later than $\mathrm{Sa}$ ) within the RA type (see also Sánchez Almeida et al. 2011).
3. While RE-type galaxies extend over the entire range of primary morphology, they are dominated by spiral galaxies (mostly earlier than Sd galaxies) including edge-on galaxies. 

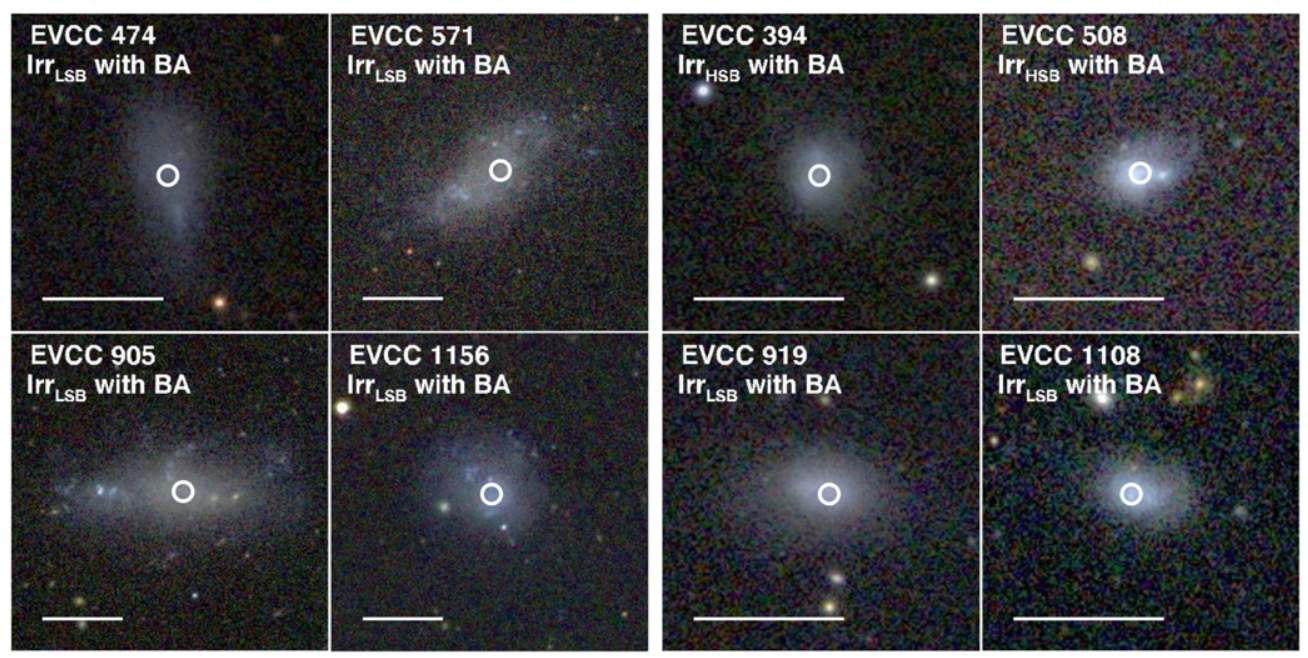

Figure 9. Examples of BA-type irregular galaxies. The open circle indicates the fiber location of the SDSS spectroscopic observation. The solid bar corresponds to $30 \operatorname{arcsec}$ on the sky. North is up and east is to the left.

(A color version of this figure is available in the online journal.)

4. Most BA-type galaxies are early-type dwarf galaxies (dE and dS0) or HSB/LSB Irr galaxies. A small fraction (5\%) of early-type dwarf galaxies are of BA type. On the other hand, this type contains only a few spiral galaxies.

5. BE-type galaxies are mostly associated with the irregular galaxies and late spiral galaxies ( $\mathrm{Sc}$ and $\mathrm{Sd}$ ). Interestingly, a considerable fraction $(14 \%)$ of early-type dwarf galaxies (dE and $\mathrm{dSO}$ ) are of BE type. Furthermore, some early-type dwarf galaxies classified as BA and BE type exhibit a blue core (see also Figure 8; Lisker et al. 2006).

6 . While the majority (86\%) of irregular galaxies are of BE type, which is the result of ongoing star formation, a fraction $(12 \%)$ of them are classified as BA type with no prominent Balmer emission line. In the case of some BA-type Irr galaxies with relatively large size and diffuse structures, SDSS fiber location, which is offset to the bright starforming knots in a galaxy, is responsible for their secondary morphology (see the left panels of Figure 9). On the other hand, some relatively compact Irrs also show BA type, although the SDSS fiber is located at the galaxy center (see the right panels of Figure 9). These galaxies have probably experienced a recent quenching of star formation.

7. A relatively dense obscuring band of interstellar dust is often detectable in optical images of spiral galaxies. Different classes of dust lanes are defined depending on the morphology and inclination of galaxies (see Buta 2013 for details). A significant fraction (72\%, e.g., EVCC 340, EVCC 488, and EVCC 634 in Figure 10) of Sa and SBa galaxies in RE type show dust lanes, while no such galaxy is seen in RA type.

8. The edge-on galaxies are dominated by RE- and BE-type galaxies. The BE-type edge-on galaxies show overall bluer colors compared to their RE-type counterparts. Furthermore, a large fraction (75\%) of RE-type edge-on galaxies exhibit dust lanes (see EVCC 574, EVCC 587, and EVCC 1036 in Figure 11).

In Figure 12, we also present a two-dimensional histogram comparing the two morphological classifications for 135 galaxies whose spectra are off center by more than 5 arcsec from the photometric galaxy center. This allows us to examine whether off-center spectra are likely to affect the secondary morpholog- ical classification. The first obvious conclusion drawn from inspection is that the overall distribution for the off-center spectra appear to be quite consistent with that for on-center spectra (see Figure 7). This denotes that within our classification scheme, the fiber location in the SDSS does not significantly affect the secondary morphology at a given primary morphology. However, note that in the case of spiral galaxies including edge-on systems with off-center spectra, they are mostly (72\%) dominated by BE type and only a small fraction (20\%) of RE-type galaxies are found contrary to the cases of on-center spectra. This is mostly because the fibers are positioned on the star-forming regions in the disk rather than the bulges.

\section{PHOTOMETRY}

While the SDSS photometric pipeline is optimized for small, faint objects, the photometric parameters for large, bright galaxies are often affected by deblending (or shredding) and imperfect sky subtraction (Abazajian et al. 2004, 2009; West et al. 2010; Blanton et al. 2011). Galaxies with large angular sizes, irregular morphology, and bright sub-clumps (e.g., H II regions and spiral arms) are often shredded into multiple, separate objects and thus the derived total flux for these galaxies are often unreliable. Furthermore, the SDSS photometric pipeline systematically underestimates the luminosities of galaxies with large size and/or in crowded fields, due to the overestimation of the sky background as they occupy a considerable fraction of the $256 \times 256$ pixel mask. In order to overcome these problems, we performed our own photometry for all galaxies in the EVCC.

The photometry of all EVCC galaxies was performed using Source Extractor (SExtractor, Bertin \& Arnouts 1996). Additionally, photometric parameters for VCC galaxies that are not in common with the EVCC were also measured. For each galaxy, we created SDSS postage images in five passbands $(u, g, r, i$, and $z$ ) depending on the angular size of the galaxy. For the construction of the background map, unlike the pipeline of the SDSS DR7, we masked out all proper objects in the image (see also Blanton et al. 2011). This allows us to eliminate any contribution of light from stars and background galaxies and then avoid overestimating of sky background regardless of the BACK_SIZE in SExtractor. In this masking image, masked regions which are defined by SExtractor segmentation image are 


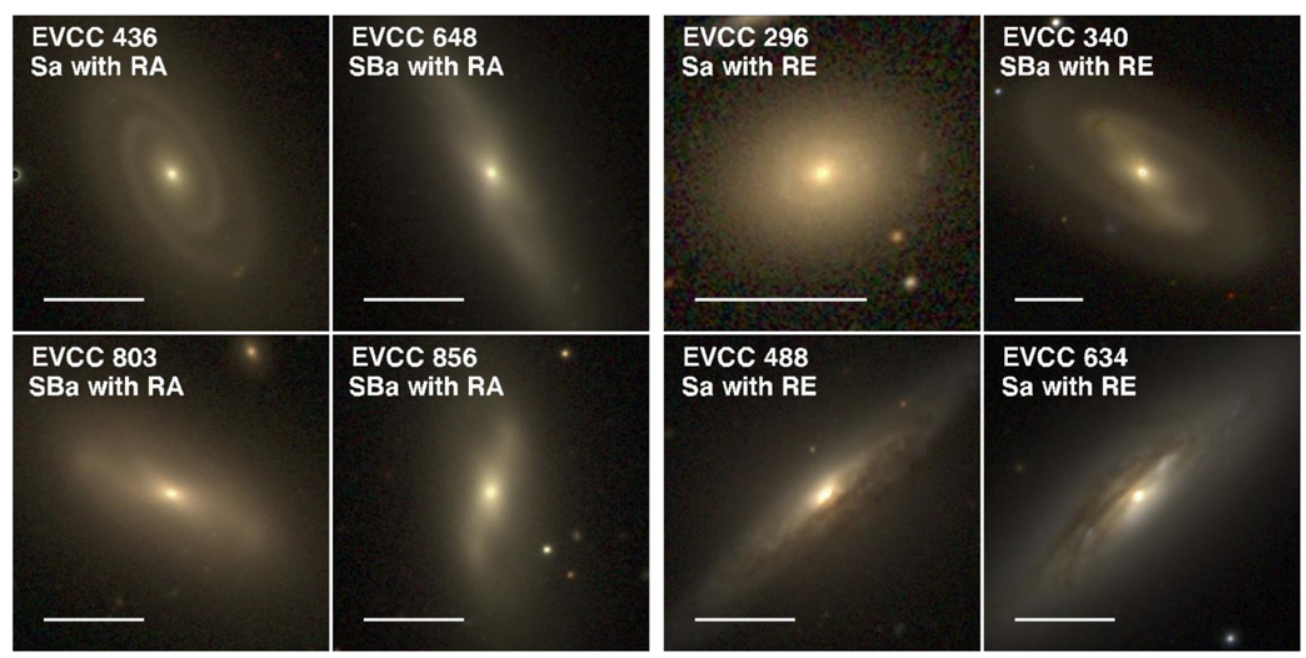

Figure 10. Examples of RA- (left panels) and RE-type (right panels) Sa and SBa galaxies. The solid bar corresponds to 30 arcsec on the sky. North is up and east is to the left.

(A color version of this figure is available in the online journal.)
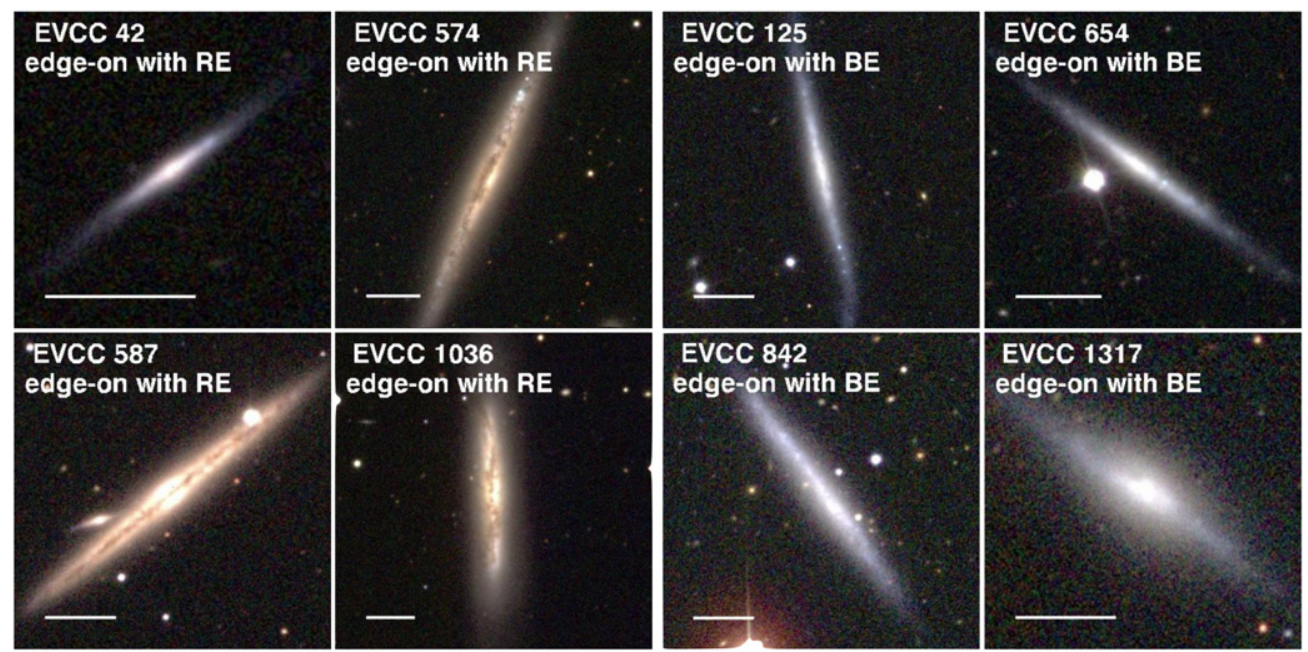

Figure 11. Examples of RE- (left panels) and BE-type (right panels) edge-on spiral galaxies. The solid bar corresponds to 30 arcsec on the sky. North is up and east is to the left.

(A color version of this figure is available in the online journal.)

filled by median value calculated from surrounding all pixels. Then, SExtractor constructs a background map by dividing the whole masking image into a grid of background meshes set by BACK_SIZE $=256$ pixels.

Source detection and deblending were carried out on the $r$-band image, because it offers the highest signal-to-noise ratio $(\mathrm{S} / \mathrm{N})$ for most sources. The resulting positional and basic shape parameters were used for subsequent photometry in the other passbands. We found that the following parameters led to optimal source detection; DETECT_THRESHOLD $=1$ and DETECT_MINAREA $=10$. In some cases where galaxies were faint or affected by neighboring bright sources, different parameters were chosen (DETECT_THRESHOLD $=0.5-1$ and DETECT_MINAREA $=5-50$ ), which were verified by visual inspection, confirming that obvious galaxies are detected and a large number of spurious detections are avoided. We selected individual values for the deblending parameters (DEBLEND_MINCONT and DEBLEND_NTHRESH) for each galaxy that minimize the fraction of shredded, spurious objects from target galaxy. The final values of parameters are selected by visual inspection of detected objects iteratively and typical values are DEBLEND_MINCONT $=0.001-0.05$ and DEBLEND_NTHRESH $=1-64$. If the deblending algorithm did not extract possible neighbor sources, we selected those by visual inspection and removed them in the image manually using the IMEDIT command in IRAF.

We adopted MAG_AUTO as the total magnitude of a galaxy, which provides the flux within $k$ (Kron factor) times the Kron radius. For the total flux, we used $k=2.5$, which is expected to recover more than $94 \%$ of the total galaxy light (Bertin \& Arnouts 1996). Once a flux was calculated, we converted all fluxes to asinh magnitudes (Lupton et al. 1999). In Figure 13, we compare our own photometry of all galaxies in the EVCC with the Petrosian magnitude from the SDSS photometric pipeline. The main trend is that the SDSS pipeline systematically underestimates the fluxes of larger galaxies. There is a difference of up to 1.5 magnitude. This confirms that the photometry from the SDSS pipeline has problems with deblending and sky subtraction for large, bright galaxies. 


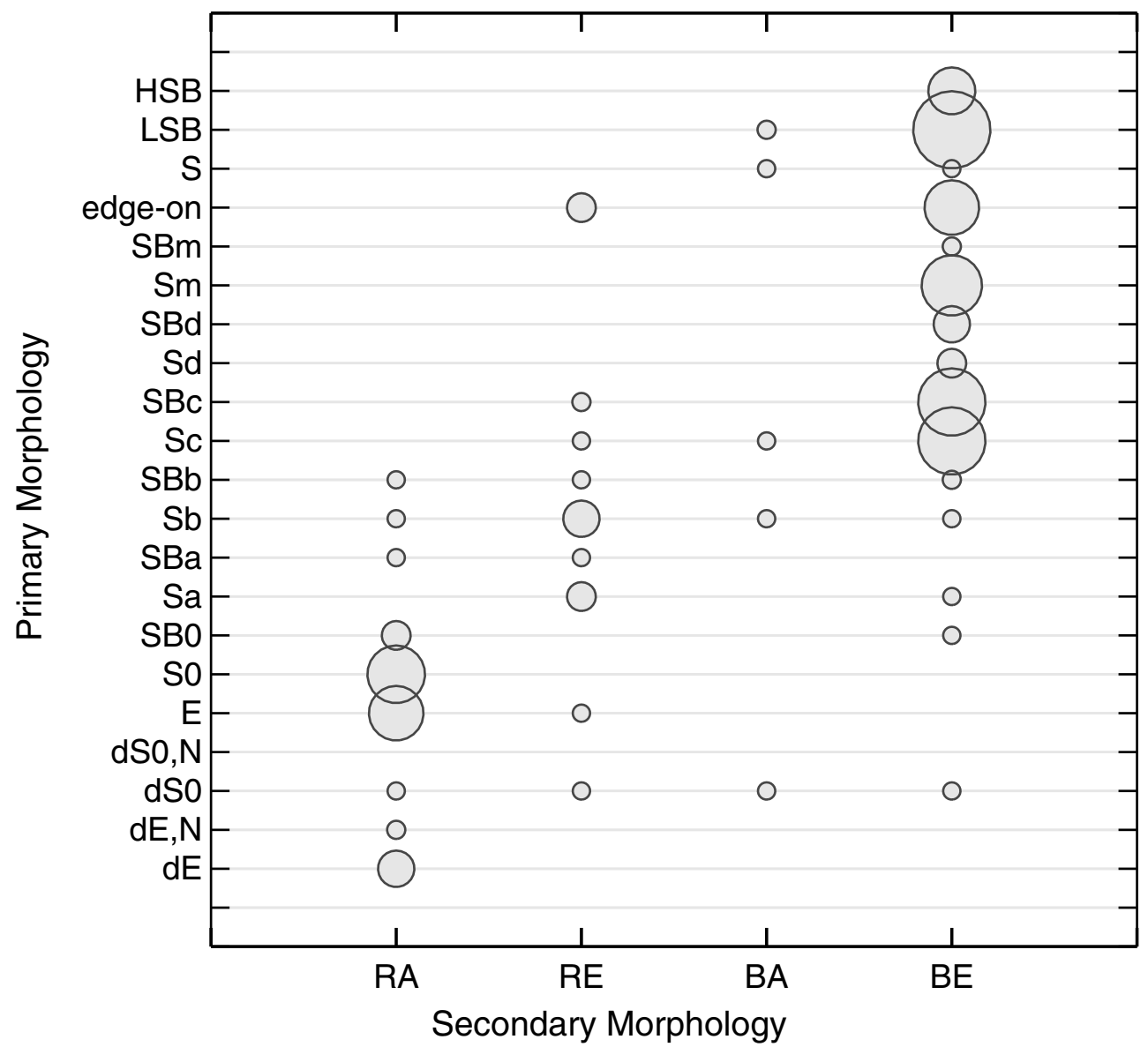

Figure 12. Comparison between primary and secondary morphology of 135 galaxies with off-center spectra in the EVCC. The fibers for these spectra are off-center by more than 5 arcsec from the photometric galaxy center. The symbol size is proportional to the number of galaxies.

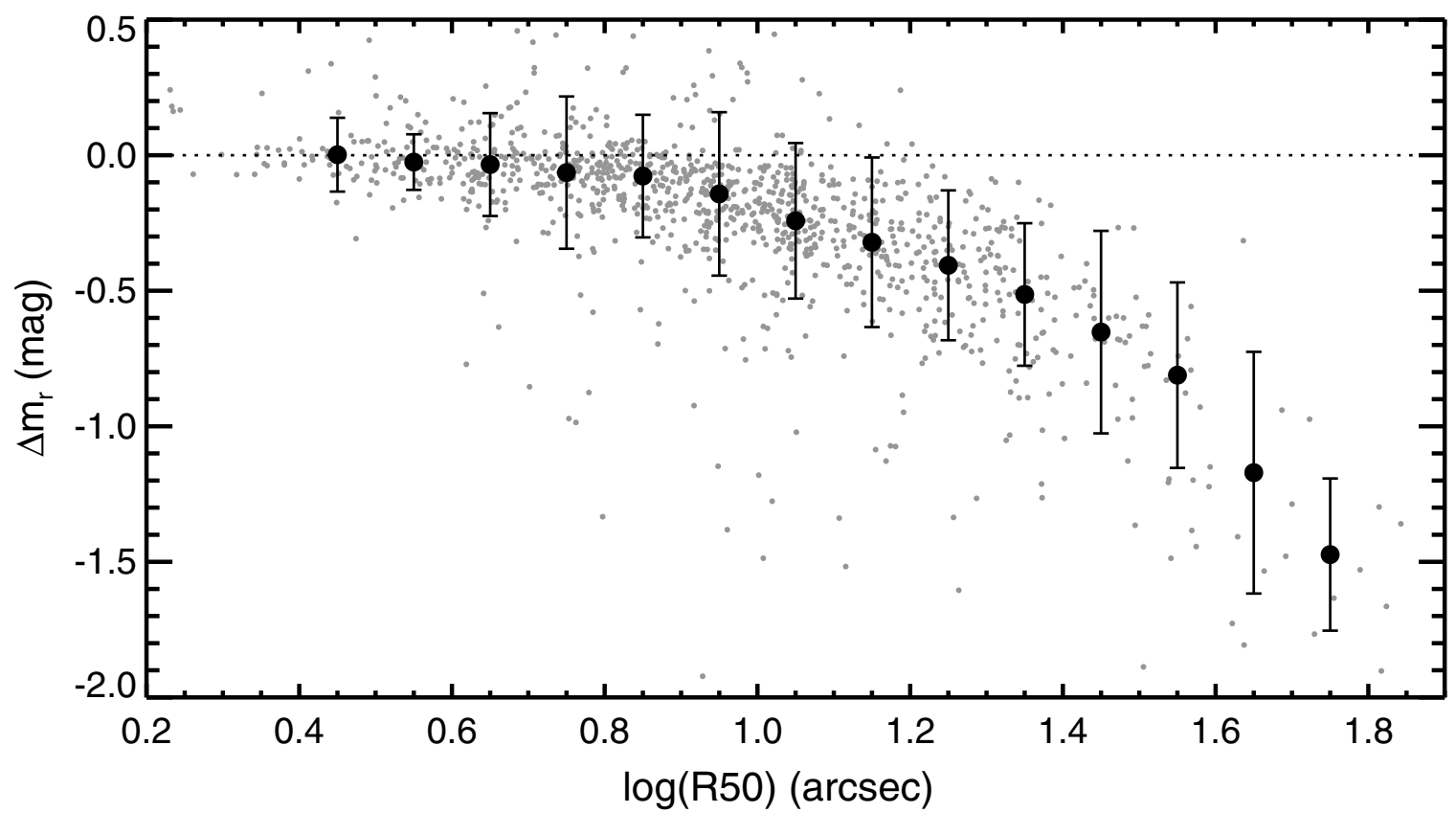

Figure 13. Difference between the galaxy's total $r$-band magnitude from our own photometry and Petrosian magnitude of the SDSS pipeline as a function of $r$-band half-light radius (R50). Filled circles and error bars denote the median value and standard deviation for every 0.1 dex interval.

Foreground Galactic extinction correction for each galaxy was then applied (Schlegel et al. 1998). We used the reddening law of Cardelli et al. (1989) to derive the following: $R_{u}=5.049$, $R_{g}=3.889, R_{r}=2.822, R_{i}=2.176$, and $R_{z}=1.567$.
Magnitude errors were estimated following the equation given by Lisker et al. (2008), where several different sources of uncertainties are added in quadrature: $\mathrm{S} / \mathrm{N}$ uncertainty, sky level uncertainty, uncertainty in the determination of semimajor 


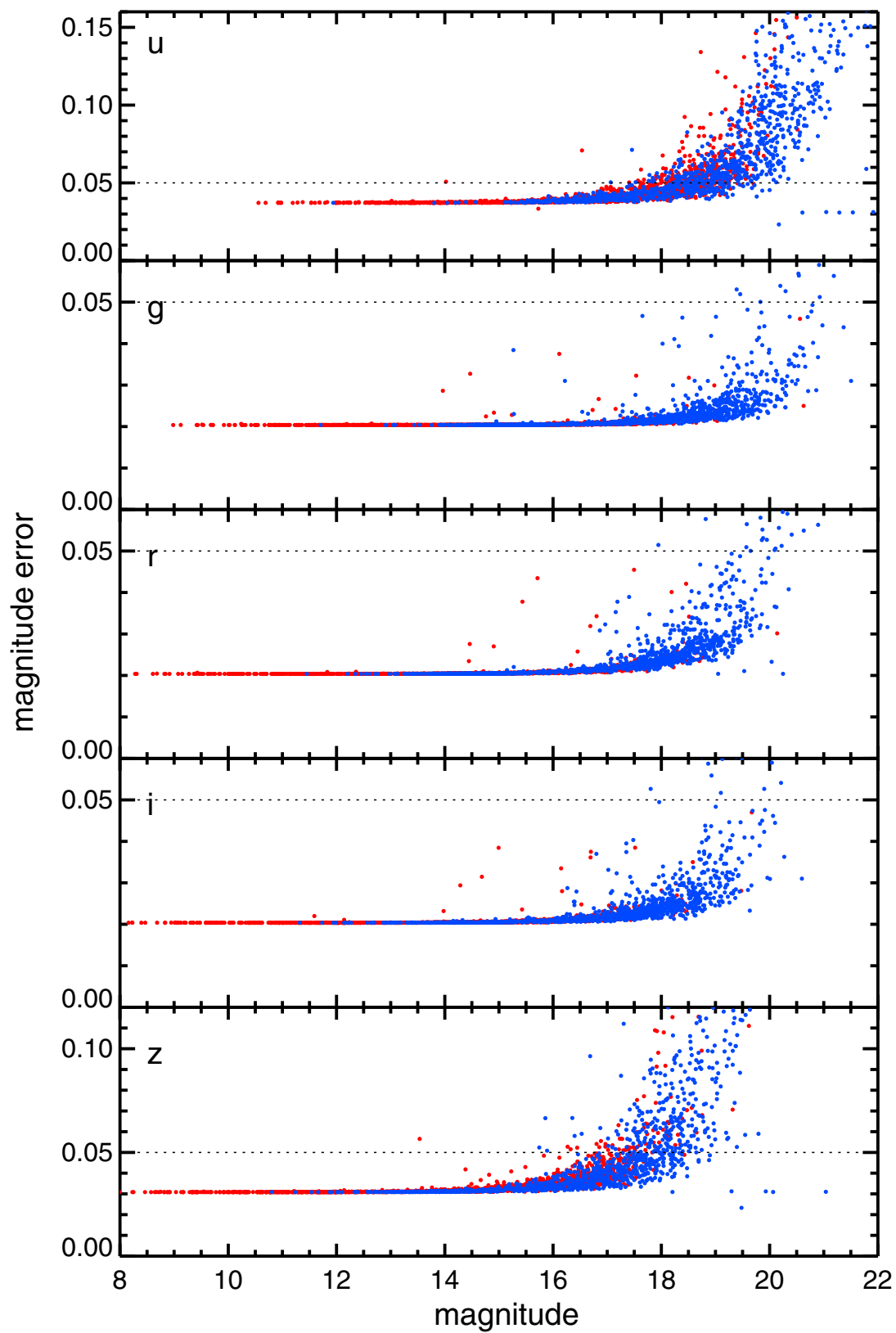

Figure 14. Magnitude error vs. total magnitude in each passband. Red and blue dots are for galaxies in the EVCC and included only in the VCC, respectively. In each panel, the dotted line indicates the magnitude error of 0.05 .

(A color version of this figure is available in the online journal.)

axis of aperture, photometric calibration uncertainty, and $u$-band leak uncertainty (see Section 4 of Lisker et al. 2008 for details). As an example, uncertainty of flux measurement for the $u$ band is given below:

$$
\begin{aligned}
\Delta f_{\mathrm{u}} / f_{\mathrm{u}}= & \left(\left(\frac{\sigma_{\mathrm{u}} \cdot \sqrt{N_{\text {pix }}}}{f_{\mathrm{u}}}\right)^{2}+2 \times\left(\frac{0.002 \sigma_{\mathrm{u}} \cdot N_{\text {pix }}}{f_{\mathrm{u}}}\right)^{2}\right. \\
& \left.+\left(10^{0.4 \times 0.03}-1\right)^{2}+\left(10^{0.4 \times 0.02}-1\right)^{2}\right)^{0.5}
\end{aligned}
$$

Here, $f_{\mathrm{u}}$ denotes the flux measurement. $\sigma_{\mathrm{u}}$ and $N_{\text {pix }}$ are the noise level per pixel and the number of pixels included in the given aperture, respectively. The first term is the $\mathrm{S} / \mathrm{N}$ uncertainty and the second term is the combination of sky level uncertainty and aperture uncertainty. The last two terms are the calibration uncertainty and the $u$-band leak uncertainty, respectively. Figure 14 presents the distribution of magnitude error for each passband.

\section{THE CATALOG}

The EVCC contains 1589 galaxies. The 23 columns in Table 2 present the full EVCC catalog including fundamental information such as membership, morphology, and photometric parameters.

Column 1: EVCC ID number. Galaxies with numbers larger than 2000 have radial velocities from the NED. ID is marked with the right ascension.

Column 2: VCC ID number.

Column 3: NGC designation. 
Column 4: Right ascension (J2000) from SExtractor in degrees.

Column 5: Declination (J2000) from SExtractor in degrees.

Column 6: Right ascension (J2000) of the SDSS fiber location in degrees.

Column 7: Declination (J2000) of the SDSS fiber location in degrees.

Column 8: Offset between the photometric center and SDSS fiber location in arcsecs.

Column 9: Heliocentric radial velocity from the SDSS in $\mathrm{km} \mathrm{s}^{-1}$.

Column 10: Heliocentric radial velocity from the NED in $\mathrm{km} \mathrm{s}^{-1}$.

Column 11: Membership of galaxy as derived from the Virgo cluster infall model $(\mathrm{M}=$ certain member, $\mathrm{P}=$ possible member; see Section 2.4).

Column 12: Membership of galaxy from the VCC $(\mathrm{M}=$ member, $\mathrm{P}=$ possible member, $\mathrm{B}=$ background galaxy).

Column 13: Primary morphology.

Column 14: Secondary morphology.

Column 15: Primary morphology in numerical type (see Table 1).

Column 16: Morphology from the VCC.

Column 17: $u$-band AUTO magnitude from SExtractor and error.

Column 18: $g$-band AUTO magnitude from SExtractor and error.

Column 19: $r$-band AUTO magnitude from SExtractor and error.

Column 20: $i$-band AUTO magnitude from SExtractor and error.

Column 21: z-band AUTO magnitude from SExtractor and error.

Column 22: $r$-band Kron radius in arcsecs.

Column 23: $r$-band half-light radius (R50) in arcsecs from SExtractor which encloses $50 \%$ of the flux.

In addition, we present in Table 3 the photometric parameters for 1183 galaxies in the VCC which are not in common with the EVCC. They either have no available radial velocity information or have radial velocity with $c z>3000 \mathrm{~km} \mathrm{~s}^{-1}$ from the SDSS and NED. The columns in Table 3 are as follows.

Column 1: VCC ID number.

Column 2: NGC designation.

Column 3: Right ascension (J2000) from SExtractor in degrees.

Column 4: Declination (J2000) from SExtractor in degrees.

Column 5: Heliocentric radial velocity from the SDSS in $\mathrm{km} \mathrm{s}^{-1}$.

Column 6: Heliocentric radial velocity from the NED in $\mathrm{km} \mathrm{s}^{-1}$.

Column 7: Membership of galaxy from the VCC $(\mathrm{M}=$ member, $\mathrm{P}=$ possible member, $\mathrm{B}=$ background galaxy).

Column 8: Morphology from the VCC.

Column 9: $u$-band AUTO magnitude from SExtractor and error.

Column 10: $g$-band AUTO magnitude from SExtractor and error.
Column 11: $r$-band AUTO magnitude from SExtractor and error.

Column 12: $i$-band AUTO magnitude from SExtractor and error.

Column 13: $z$-band AUTO magnitude from SExtractor and error.

Column 14: $r$-band Kron radius in arcsecs.

Column 15: $r$-band half-light radius (R50) in arcsecs from SExtractor which encloses $50 \%$ of the flux.

\section{COMPARISON BETWEEN THE EVCC AND VCC}

In the following, we compare the EVCC and VCC in terms of morphology, projected spatial distribution, and galaxy luminosity function. For that purpose we have arranged the morphologies of the galaxies in the two catalogs in a consistent way. VCC galaxies with mixed morphologies (e.g., dE/dS0, dE/ $\mathrm{Im}, \mathrm{Im} / \mathrm{BCD}$, etc; Binggeli et al. 1985) have been assigned to the first morphology class. For VCC galaxies with uncertain morphologies, indicated by ":" or "?” (e.g., dE:, dE?, etc; Binggeli et al. 1985), we take their morphologies at face value. The Im and BCD galaxies in the VCC approximately correspond to the $\operatorname{Irr}(\mathrm{LSB})$ - and $\operatorname{Irr}(\mathrm{HSB})$-type galaxies in the EVCC, respectively. In addition, we do not discriminate between barred and non-barred galaxies in both cases of the VCC and EVCC (e.g., from dSB0 and $\mathrm{SBc}$ to $\mathrm{dS} 0$ and $\mathrm{Sc}$, respectively).

\subsection{Comparison of Morphology}

In Figure 15, we present a two-dimensional histogram comparing the morphologies for the 913 EVCC galaxies that are in common with the VCC. Our morphological classification in the EVCC is generally consistent with the VCC classification. There is a close trend along the diagonal between two catalogs. The differences are typically at the level of one or two morphological subtypes. The morphological classifications of $90 \%$ (822 of 913) galaxies are in good agreement within deviation of two morphological types.

However, it is worth noting that the relationship has some scatter at a fixed morphological type. In the following, we discuss the most interesting outliers, where the morphological classification between our EVCC and the VCC disagree more significantly.

1. Significant disagreements occur among the early-type dwarf galaxies (i.e., dE, dE(N), dS0, and dSO(N)) for each catalog. Some early-type dwarf galaxies in the EVCC are classified as galaxies with late-type (i.e., BCD and $\mathrm{Im}$ ) or unknown morphologies in the VCC (see the upper left part of Figure 15). All of these galaxies exhibit elliptical shapes with overall smooth and regular appearances (see Figure 16). Although at first glance galaxies like EVCC 403, EVCC 730, EVCC 836, EVCC 851, and EVCC 1004 seem to have a central star-forming region, using an alternating color scheme to display the $r$-band image reveals smooth, elliptical isophotes in the center. Other galaxies like EVCC 464, EVCC 806, and EVCC 832 in Figure 16 seem to host many small, compact star-forming regions. However, these star-forming regions turned out to be faint foreground stars and background galaxies mostly with red colors judging from visual inspection of the color images.

The opposite situation is also seen: some early-type dwarf galaxies in the VCC have been classified as 
The Extended Virgo Cluster Catalog ${ }^{\mathrm{a}}$

\begin{tabular}{|c|c|c|c|c|c|c|c|c|c|c|c|c|c|c|c|c|c|c|c|c|c|c|}
\hline $\begin{array}{l}\text { ID } \\
\text { EVCC }\end{array}$ & $\begin{array}{c}\text { ID } \\
\text { VCC }\end{array}$ & NGC & $\begin{array}{l}\text { R.A. } \\
\text { (deg) } \\
(4)\end{array}$ & $\begin{array}{l}\text { decl. } \\
\text { (deg) } \\
(5)\end{array}$ & $\begin{array}{l}\text { R.A. } \\
\text { Fiber } \\
\text { (deg) } \\
(6)\end{array}$ & $\begin{array}{l}\text { decl. } \\
\text { Fiber } \\
(\mathrm{deg}) \\
(7)\end{array}$ & $\begin{array}{c}(\operatorname{arcsec}) \\
(8)\end{array}$ & $\begin{array}{c}\mathrm{cz} \\
\mathrm{SDSS} \\
\left(\mathrm{km} \mathrm{s}^{-1}\right) \\
(9)\end{array}$ & $\begin{array}{c}\mathrm{cz} \\
\mathrm{NED} \\
\left(\mathrm{km} \mathrm{s}^{-1}\right) \\
(10)\end{array}$ & $\begin{array}{r}\text { Memb } \\
\text { EVCC } \\
\text { (11) }\end{array}$ & $\begin{array}{c}\text { Memb } \\
\text { VCC } \\
(12)\end{array}$ & $\begin{array}{c}\text { Morp } \\
\text { Primary } \\
(13)\end{array}$ & $\begin{array}{c}\text { Morp } \\
\text { Secondary } \\
\text { (14) }\end{array}$ & $\begin{array}{l}\text { Morp } \\
\text { index }\end{array}$ & $\begin{array}{l}\text { Morp } \\
\text { VCC } \\
(16)\end{array}$ & $\begin{array}{c}u \\
\text { AUTO } \\
(\mathrm{mag}) \\
(17)\end{array}$ & $\begin{array}{c}g \\
\text { AUTO } \\
(\mathrm{mag}) \\
(18)\end{array}$ & $\begin{array}{c}r \\
\text { AUTO } \\
(\mathrm{mag}) \\
(19)\end{array}$ & $\begin{array}{c}i \\
\text { AUTO } \\
(\mathrm{mag}) \\
(20)\end{array}$ & $\begin{array}{c}z \\
\text { AUTO } \\
(\mathrm{mag}) \\
(21)\end{array}$ & $\begin{array}{c}R_{\mathrm{Kron}} \\
(\operatorname{arcsec}) \\
(22)\end{array}$ & $\begin{array}{c}\text { R50 } \\
(\operatorname{arcsec}) \\
(23)\end{array}$ \\
\hline 0001 & $\cdots$ & . & 175.0771 & 9.0099 & 175.0771 & 9.0099 & 0.241 & 1843.7 & 1860 & $\mathrm{P}$ & $\cdots$ & $\mathrm{Sa}$ & RE & 201 & $\cdots$ & $14.95 \pm 0.037$ & $13.50 \pm 0.020$ & $12.75 \pm 0.020$ & $12.33 \pm 0.020$ & $12.01 \pm 0.030$ & 50.01 & 22.44 \\
\hline 0002 & $\ldots$ & $\ldots$ & 175.2361 & 14.0744 & 175.2364 & 14.0742 & 1.346 & 977.9 & $\ldots$ & M & $\ldots$ & $\mathrm{S}$ & $\mathrm{BE}$ & 206 & $\ldots$ & $17.32 \pm 0.039$ & $16.15 \pm 0.020$ & $15.81 \pm 0.020$ & $15.49 \pm 0.020$ & $15.13 \pm 0.032$ & 36.20 & 19.15 \\
\hline 0003 & $\ldots$ & 3801 & 175.2447 & 11.4710 & 175.2448 & 11.4711 & 0.477 & 999.3 & 992 & M & $\cdots$ & $\mathrm{Sc}$ & $\mathrm{RE}$ & 203 & $\cdots$ & $12.13 \pm 0.038$ & $11.04 \pm 0.020$ & $10.50 \pm 0.020$ & $10.24 \pm 0.020$ & $10.01 \pm 0.031$ & 135.68 & 85.93 \\
\hline 0004 & $\ldots$ & $\ldots$ & 175.4609 & 15.9736 & 175.4610 & 15.9737 & 0.468 & 753.1 & 748 & M & $\ldots$ & $\operatorname{Irr}(\mathrm{HSB})$ & $\mathrm{BE}$ & & $\ldots$ & $15.50 \pm 0.037$ & $14.59 \pm 0.020$ & $14.12 \pm 0.020$ & $13.90 \pm 0.020$ & $13.76 \pm 0.030$ & 26.01 & 14.96 \\
\hline 0005 & $\ldots$ & $\ldots$ & 175.5747 & 14.9948 & 175.5792 & 14.9962 & 16.601 & 1021.5 & 1024 & M & $\ldots$ & $\operatorname{Irr}(\mathrm{LSB})$ & $\mathrm{BE}$ & 300 & $\ldots$ & $16.33 \pm 0.038$ & $15.54 \pm 0.020$ & $15.41 \pm 0.020$ & $15.08 \pm 0.020$ & $15.52 \pm 0.036$ & 106.17 & 56.61 \\
\hline 0006 & $\ldots$ & $\cdots$ & 175.6223 & 18.3326 & 175.6227 & 18.3325 & 1.503 & 906.4 & 922 & M & $\cdots$ & $\mathrm{Sc}$ & $\mathrm{BE}$ & 203 & $\cdots$ & $14.14 \pm 0.037$ & $13.32 \pm 0.020$ & $12.99 \pm 0.020$ & $12.83 \pm 0.020$ & $12.92 \pm 0.031$ & 99.26 & 53.93 \\
\hline 0007 & $\ldots$ & $\ldots$ & 175.8163 & -1.3950 & 75.8171 & -1.3946 & 3.218 & 1891.6 & $\cdots$ & $\mathrm{P}$ & $\ldots$ & $\operatorname{Irr}(\mathrm{LSB})$ & & & $\ldots$ & $17.35 \pm 0.040$ & $16.83 \pm 0.020$ & $16.56 \pm 0.021$ & $16.36 \pm 0.021$ & $16.26 \pm 0.035$ & 1.04 & 13.11 \\
\hline 0008 & $\ldots$ & $\cdots$ & 175.8624 & 11.3985 & 175.8624 & 11.3984 & 0.315 & 901.0 & $\ldots$ & M & $\cdots$ & $\operatorname{Irr}(\mathrm{HSB})$ & & 310 & $\cdots$ & $17.05 \pm 0.038$ & $16.26 \pm 0.020$ & $15.96 \pm 0.020$ & $15.81 \pm 0.020$ & $15.79 \pm 0.032$ & 17.41 & 12.17 \\
\hline 0009 & $\cdots$ & $\ldots$ & 175.9422 & 13.7076 & 175.9421 & 13.7075 & 0.238 & 2924.3 & 2920 & $P$ & $\cdots$ & S0 & $\mathrm{BE}$ & 200 & $\ldots$ & $17.76 \pm 0.040$ & $16.46 \pm 0.020$ & $15.74 \pm 0.020$ & $15.43 \pm 0.020$ & $15.23 \pm 0.031$ & 13.92 & 7.13 \\
\hline 0010 & $\ldots$ & $\cdots$ & 176.0439 & -3.6689 & 176.0441 & -3.6686 & 1.303 & 1029.1 & $\ldots$ & $\mathrm{P}$ & $\cdots$ & $\operatorname{Irr}(\mathrm{LSB})$ & & & $\ldots$ & $18.37 \pm 0.045$ & $17.28 \pm 0.020$ & $16.99 \pm 0.021$ & $16.98 \pm 0.022$ & $17.09 \pm 0.053$ & 8.26 & 14.60 \\
\hline 0011 & $\ldots$ & $\cdots$ & 176.1337 & 6.7041 & 176.1336 & 6.7040 & 0.522 & 1455.2 & $\ldots$ & $\mathrm{P}$ & $\ldots$ & $\operatorname{Irr}(\mathrm{HSB})$ & & 310 & $\ldots$ & $18.41 \pm 0.044$ & $17.49 \pm 0.021$ & $17.20 \pm 0.021$ & $16.97 \pm 0.021$ & $16.99 \pm 0.040$ & 1.87 & 11.51 \\
\hline 0012 & $\ldots$ & & 176.1515 & 19.4781 & 176.1516 & 19.4776 & 1.844 & 2775.5 & $\ldots$ & $\mathrm{P}$ & $\ldots$ & $\operatorname{Irr}(\mathrm{L}$ & & 30 & $\ldots$ & $18.43 \pm 0.042$ & $17.63 \pm 0.020$ & $17.20 \pm 0.020$ & $17.20 \pm 0.021$ & $17.05 \pm 0.044$ & 8.23 & 20.21 \\
\hline 0013 & $\ldots$ & $\ldots$ & 176.1697 & 16.8995 & 176.1696 & 16.8996 & 0.474 & 902.1 & $\cdots$ & M & $\ldots$ & dS0 & B & 410 & $\ldots$ & $18.40 \pm 0.054$ & $16.72 \pm 0.020$ & $16.25 \pm 0.020$ & $16.11 \pm 0.020$ & $15.37 \pm 0.032$ & 16.30 & 10.91 \\
\hline 0014 & $\ldots$ & $\ldots$ & 176.1809 & 11.2071 & 176.1811 & 11.2073 & 0.836 & 2944.3 & $\cdots$ & $\mathrm{P}$ & $\ldots$ & $\operatorname{Irr}(\mathrm{LSB})$ & B & 300 & $\ldots$ & $17.93 \pm 0.042$ & $17.00 \pm 0.020$ & $16.75 \pm 0.021$ & $16.70 \pm 0.021$ & $16.63 \pm 0.042$ & 3.66 & 15.33 \\
\hline 0015 & $\ldots$ & $\ldots$ & 176.3174 & 13.8726 & 176.3174 & 13.8725 & 0.454 & 2974.0 & $\ldots$ & $\mathrm{P}$ & $\ldots$ & $\mathrm{dE}$ & RA & 400 & $\ldots$ & $19.37 \pm 0.055$ & $18.20 \pm 0.022$ & $17.50 \pm 0.022$ & $17.23 \pm 0.021$ & $16.94 \pm 0.034$ & 3.37 & 9.14 \\
\hline 0016 & $\ldots$ & 3876 & 176.3609 & 9.1610 & 176.3620 & 9.1637 & 10.258 & 2862.8 & 2892 & $\mathrm{P}$ & $\ldots$ & $\mathrm{SBb}$ & $\mathrm{BE}$ & 21 & $\ldots$ & $14.48 \pm 0.037$ & $13.52 \pm 0.020$ & $13.19 \pm 0.020$ & $12.98 \pm 0.074$ & $12.83 \pm 0.030$ & 42.55 & 25.34 \\
\hline 0017 & $\ldots$ & $\ldots$ & 176.4739 & 9.8998 & 176.4740 & 9.8997 & 0.444 & 2918.5 & $\ldots$ & $\mathrm{P}$ & $\ldots$ & dS0 & $\mathrm{BE}$ & 410 & $\ldots$ & $18.51 \pm 0.044$ & $17.26 \pm 0.020$ & $16.93 \pm 0.021$ & $16.76 \pm 0.021$ & $16.50 \pm 0.038$ & 13.72 & 7.88 \\
\hline 0018 & $\ldots$ & $\ldots$ & 176.5169 & 11.5813 & 176.5168 & 11.5813 & & 2970.2 & 2969 & & $\ldots$ & So & & & $\ldots$ & $16.47 \pm 0.037$ & $15.47 \pm 0.020$ & $15.00 \pm 0.020$ & $14.75 \pm 0.020$ & $14.61 \pm 0.031$ & 7.35 & 8.74 \\
\hline 0019 & $\ldots$ & $\ldots$ & 176.7524 & -0.2939 & 176.7530 & -0.2942 & 2.442 & 1463.1 & 1478 & $\mathrm{P}$ & $\cdots$ & dS0 & & 410 & $\cdots$ & $16.24 \pm 0.037$ & $15.10 \pm 0.020$ & $14.60 \pm 0.020$ & $14.34 \pm 0.020$ & $14.21 \pm 0.031$ & 33.48 & 17.23 \\
\hline 0020 & $\ldots$ & $\ldots$ & 176.7792 & 3.1064 & 176.7791 & 3.1064 & 0.232 & 1012.7 & $\ldots$ & M & $\ldots$ & $\mathrm{dE}$ & $\mathrm{BE}$ & 400 & $\ldots$ & $18.63 \pm 0.044$ & $17.69 \pm 0.020$ & $17.36 \pm 0.021$ & $17.06 \pm 0.021$ & $16.72 \pm 0.035$ & 17.45 & 11.48 \\
\hline 0021 & $\ldots$ & $\ldots$ & 176.8230 & 16.3346 & 176.8232 & 16.3345 & 0.593 & & $\ldots$ & M & $\ldots$ & $\mathrm{dE}$ & & 400 & $\ldots$ & $18.53 \pm 0.045$ & $17.70 \pm 0.021$ & $17.29 \pm 0.022$ & $16.96 \pm 0.021$ & $17.09 \pm 0.039$ & 16.99 & 10.04 \\
\hline 0022 & $\ldots$ & $\ldots$ & 177.0440 & -1.9892 & 177.0443 & -1.9891 & & 1529.3 & $\ldots$ & $\mathrm{P}$ & $\cdots$ & $\operatorname{Irr}(\mathrm{LSB})$ & & & $\ldots$ & $17.54 \pm 0.040$ & $16.81 \pm 0.020$ & $16.64 \pm 0.021$ & $16.65 \pm 0.021$ & $16.46 \pm 0.039$ & 24.45 & 16.41 \\
\hline 0023 & $\ldots$ & $\ldots$ & 177.0683 & 18.6423 & 177.0680 & 18.6425 & 1.145 & 1010.6 & $\ldots$ & M & $\ldots$ & $\operatorname{Irr}(\mathrm{LSB})$ & $\mathrm{BE}$ & 300 & $\ldots$ & $17.13 \pm 0.039$ & $16.37 \pm 0.020$ & $16.11 \pm 0.020$ & $15.89 \pm 0.020$ & $15.52 \pm 0.033$ & 30.68 & 20.85 \\
\hline 0024 & $\ldots$ & $\ldots$ & 177.1789 & 17.1811 & 177.1797 & 17.1814 & 2.860 & 1058.7 & $\ldots$ & M & $\ldots$ & $\operatorname{Irr}(\mathrm{LSB})$ & B & 300 & $\ldots$ & $17.89 \pm 0.043$ & $17.11 \pm 0.020$ & $16.80 \pm 0.021$ & $16.60 \pm 0.021$ & $16.83 \pm 0.041$ & 27.44 & 17.21 \\
\hline 0025 & $\ldots$ & $\ldots$ & 177.2101 & -2.0326 & 177.2100 & -2.0322 & 1.527 & 1712.5 & 1723 & $\mathrm{P}$ & $\ldots$ & $\mathrm{Sc}$ & $\mathrm{BE}$ & 203 & $\ldots$ & $14.57 \pm 0.037$ & $13.67 \pm 0.020$ & $13.34 \pm 0.020$ & $12.91 \pm 0.020$ & $13.02 \pm 0.031$ & 184.37 & 89.82 \\
\hline
\end{tabular}

Note. ${ }^{\text {a }}$ The catalog of the EVCC will be updated at http://sites.google.com/site/extendedvcc/.

(This table is available in its entirety in a machine-readable form in the online journal. A portion is shown here for guidance regarding its form and content.) 
Galaxies in the VCC

\begin{tabular}{|c|c|c|c|c|c|c|c|c|c|c|c|c|c|c|}
\hline $\begin{array}{l}\text { ID } \\
\text { VCC }\end{array}$ & NGC & $\begin{array}{l}\text { (deg) } \\
\text { (3) }\end{array}$ & $\begin{array}{l}\text { decl. } \\
(\mathrm{deg}) \\
(4)\end{array}$ & $\begin{array}{c}\mathrm{cz} \\
\mathrm{SDSS} \\
\left(\mathrm{km} \mathrm{s}^{-1}\right) \\
(5)\end{array}$ & $\begin{array}{c}\mathrm{cz} \\
\mathrm{NED} \\
\left(\mathrm{km} \mathrm{s}^{-1}\right) \\
(6)\end{array}$ & $\begin{array}{l}\text { Memb } \\
\text { VCC }\end{array}$ & $\begin{array}{l}\text { Morp } \\
\text { VCC }\end{array}$ & $\begin{array}{c}u \\
\text { AUTO } \\
(\mathrm{mag}) \\
(9)\end{array}$ & $\begin{array}{c}g \\
\text { AUTO } \\
(\mathrm{mag}) \\
(10)\end{array}$ & $\begin{array}{c}r \\
\text { AUTO } \\
(\mathrm{mag}) \\
(11)\end{array}$ & $\begin{array}{c}i \\
\text { AUTO } \\
(\mathrm{mag}) \\
(12)\end{array}$ & $\begin{array}{c}z \\
\text { AUTO } \\
(\mathrm{mag}) \\
(13)\end{array}$ & $\begin{array}{c}R_{\mathrm{Kron}} \\
(\operatorname{arcsec}) \\
(14)\end{array}$ & $\begin{array}{c}(\operatorname{arcsec}) \\
(15)\end{array}$ \\
\hline 2 & $\cdots$ & 182.1055 & 13.8284 & $\cdots$ & $\cdots$ & M & dE2: & $18.89 \pm 0.050$ & $17.82 \pm 0.022$ & $17.37 \pm 0.022$ & $16.91 \pm 0.021$ & $17.19 \pm 0.049$ & 24.52 & 8.07 \\
\hline 3 & $\cdots$ & 182.1121 & 13.5238 & 6774.8 & 6805.8 & $\mathrm{P}$ & $\mathrm{BCD}$ ? & $17.56 \pm 0.039$ & $16.89 \pm 0.021$ & $16.67 \pm 0.021$ & $16.54 \pm 0.021$ & $16.49 \pm 0.034$ & 16.24 & 4.08 \\
\hline 5 & $\cdots$ & 182.1409 & 15.1188 & $\ldots$ & $\ldots$ & M & $\mathrm{dE} 4$ & $19.01 \pm 0.054$ & $18.09 \pm 0.021$ & $17.31 \pm 0.022$ & $16.76 \pm 0.022$ & $17.42 \pm 0.071$ & 26.12 & 7.38 \\
\hline 6 & $\cdots$ & 182.2152 & 9.1316 & 8172.0 & 8180.7 & B & $\mathrm{SBa}$ & $16.62 \pm 0.038$ & $15.40 \pm 0.020$ & $14.84 \pm 0.020$ & $14.57 \pm 0.020$ & $14.35 \pm 0.031$ & 21.16 & 5.67 \\
\hline 7 & $\cdots$ & 182.3274 & 11.4301 & 18904.5 & 18900.3 & B & $\mathrm{SBc}(\mathrm{s}) \mathrm{I}$ & $17.04 \pm 0.040$ & $15.75 \pm 0.021$ & $15.18 \pm 0.021$ & $14.84 \pm 0.021$ & $14.61 \pm 0.031$ & 22.73 & 7.64 \\
\hline 8 & $\cdots$ & 182.3373 & 13.5260 & $\cdots$ & $\cdots$ & M & dE0 & $19.94 \pm 0.089$ & $18.56 \pm 0.022$ & $18.12 \pm 0.024$ & $17.91 \pm 0.024$ & $17.47 \pm 0.040$ & 18.51 & 6.68 \\
\hline 11 & $\cdots$ & 182.3987 & 6.7425 & $\cdots$ & $\cdots$ & $\mathrm{M}$ & dE6 & $18.21 \pm 0.050$ & $16.75 \pm 0.021$ & $16.48 \pm 0.021$ & $16.02 \pm 0.021$ & $16.29 \pm 0.037$ & 50.96 & 13.88 \\
\hline 12 & $\cdots$ & 182.4348 & 12.1258 & 8643.3 & 8666.1 & B & $\mathrm{SBa}(\mathrm{s})$ & $16.96 \pm 0.039$ & $15.41 \pm 0.020$ & $14.64 \pm 0.020$ & $14.25 \pm 0.020$ & $13.96 \pm 0.031$ & 25.50 & 4.39 \\
\hline 13 & $\cdots$ & 182.4411 & 13.5520 & $\ldots$ & $\ldots$ & $\mathrm{P}$ & $?$ & $19.31 \pm 0.060$ & $18.17 \pm 0.021$ & $17.76 \pm 0.022$ & $17.46 \pm 0.021$ & $17.00 \pm 0.037$ & 27.87 & 9.38 \\
\hline 14 & $\ldots$ & 182.4631 & 11.2567 & 17931.9 & 17930.1 & $\mathrm{P}$ & BCD? & $17.73 \pm 0.041$ & $16.73 \pm 0.021$ & $16.34 \pm 0.021$ & $16.07 \pm 0.021$ & $15.96 \pm 0.033$ & 10.30 & 2.79 \\
\hline 16 & $\ldots$ & 182.5047 & 14.6155 & 6806.4 & 6774.6 & $\mathrm{P}$ & ImIII? & $17.92 \pm 0.043$ & $16.97 \pm 0.021$ & $16.58 \pm 0.021$ & $16.42 \pm 0.022$ & $16.40 \pm 0.043$ & 23.12 & 6.81 \\
\hline 18 & $\cdots$ & 182.5496 & 12.3255 & 8755.7 & 8759.1 & $\mathrm{P}$ & $\mathrm{Sc}(\mathrm{s}) \mathrm{II}$ & $16.13 \pm 0.038$ & $14.92 \pm 0.020$ & $14.36 \pm 0.020$ & $14.02 \pm 0.020$ & $13.70 \pm 0.031$ & 36.17 & 7.15 \\
\hline 19 & $\cdots$ & 182.5575 & 13.1880 & 6807.6 & 6807.6 & $\mathrm{P}$ & $\mathrm{BCD}$ ? & $17.65 \pm 0.045$ & $16.00 \pm 0.020$ & $15.15 \pm 0.020$ & $14.65 \pm 0.020$ & $14.36 \pm 0.031$ & 20.38 & 5.01 \\
\hline 20 & $\ldots$ & 182.5787 & 12.3297 & $\ldots$ & $\ldots$ & $\mathrm{P}$ & $?$ & $18.91 \pm 0.059$ & $17.85 \pm 0.021$ & $17.42 \pm 0.022$ & $16.97 \pm 0.021$ & $16.72 \pm 0.037$ & 20.09 & 5.78 \\
\hline 23 & $\cdots$ & 182.6040 & 13.3663 & $\cdots$ & $\cdots$ & $\mathrm{P}$ & dE5? & $20.22 \pm 0.138$ & $19.04 \pm 0.026$ & $17.88 \pm 0.023$ & $17.97 \pm 0.024$ & $18.29 \pm 0.065$ & 22.80 & 7.23 \\
\hline 27 & $\cdots$ & 182.6744 & 13.3311 & 6828.6 & 6819.6 & B & $\mathrm{SBc}(\mathrm{s}) \mathrm{I}$ & $15.93 \pm 0.038$ & $14.61 \pm 0.020$ & $14.07 \pm 0.020$ & $13.79 \pm 0.020$ & $13.58 \pm 0.031$ & 48.49 & 9.90 \\
\hline 28 & $\ldots$ & 182.6894 & 15.8651 & 6391.3 & 6329.4 & B & $\mathrm{SBc}$ & $16.78 \pm 0.040$ & $15.71 \pm 0.020$ & $15.29 \pm 0.021$ & $15.17 \pm 0.021$ & $15.11 \pm 0.032$ & 33.47 & 8.96 \\
\hline 35 & $\cdots$ & 182.8312 & 11.9099 & $\cdots$ & $\ldots$ & $\mathrm{P}$ & $?$ & $20.49 \pm 0.110$ & $19.52 \pm 0.023$ & $19.34 \pm 0.032$ & $18.69 \pm 0.025$ & $18.02 \pm 0.040$ & 9.34 & 3.23 \\
\hline 36 & $\cdots$ & 182.8677 & 13.5838 & $\cdots$ & $\cdots$ & $\mathrm{M}$ & dE0: & $19.50 \pm 0.067$ & $18.70 \pm 0.022$ & $18.14 \pm 0.024$ & $17.65 \pm 0.022$ & $17.97 \pm 0.047$ & 16.13 & 5.59 \\
\hline 38 & $\cdots$ & 182.9489 & 12.1436 & 4010.6 & 4022.7 & B & $\mathrm{Sc}(\mathrm{s}) \mathrm{II}-\mathrm{III}$ & $15.42 \pm 0.038$ & $14.24 \pm 0.020$ & $13.70 \pm 0.020$ & $13.39 \pm 0.020$ & $13.18 \pm 0.031$ & 63.68 & 15.58 \\
\hline 39 & $\cdots$ & 182.9974 & 15.4013 & 7111.2 & 7110.0 & B & Sbc(on_edge) & $16.11 \pm 0.039$ & $14.31 \pm 0.020$ & $13.45 \pm 0.020$ & $13.01 \pm 0.020$ & $12.69 \pm 0.031$ & 53.04 & 10.04 \\
\hline 40 & $\cdots$ & 183.0133 & 14.9046 & 6885.7 & 6953.7 & B & Sbc(s) & $16.83 \pm 0.040$ & $15.73 \pm 0.020$ & $15.20 \pm 0.021$ & $14.93 \pm 0.021$ & $14.73 \pm 0.031$ & 29.45 & 9.48 \\
\hline 42 & $\cdots$ & 183.0252 & 14.9529 & $\ldots$ & $\ldots$ & M & dE0 & $19.39 \pm 0.053$ & $18.95 \pm 0.023$ & $18.22 \pm 0.023$ & $17.88 \pm 0.022$ & $17.61 \pm 0.039$ & 24.50 & 8.87 \\
\hline 43 & 4164 & 183.0228 & 13.2056 & 19467.7 & 17512.2 & $\mathrm{P}$ & E3 & $16.74 \pm 0.044$ & $14.95 \pm 0.020$ & $13.95 \pm 0.020$ & $13.67 \pm 0.020$ & $13.40 \pm 0.031$ & 77.83 & 16.16 \\
\hline
\end{tabular}

Note. ${ }^{a}$ The VCC galaxies which are not in common with the EVCC. The catalog of the EVCC will be updated at http://sites.google.com/site/extendedvcc/.

(This table is available in its entirety in a machine-readable form in the online journal. A portion is shown here for guidance regarding its form and content.) 


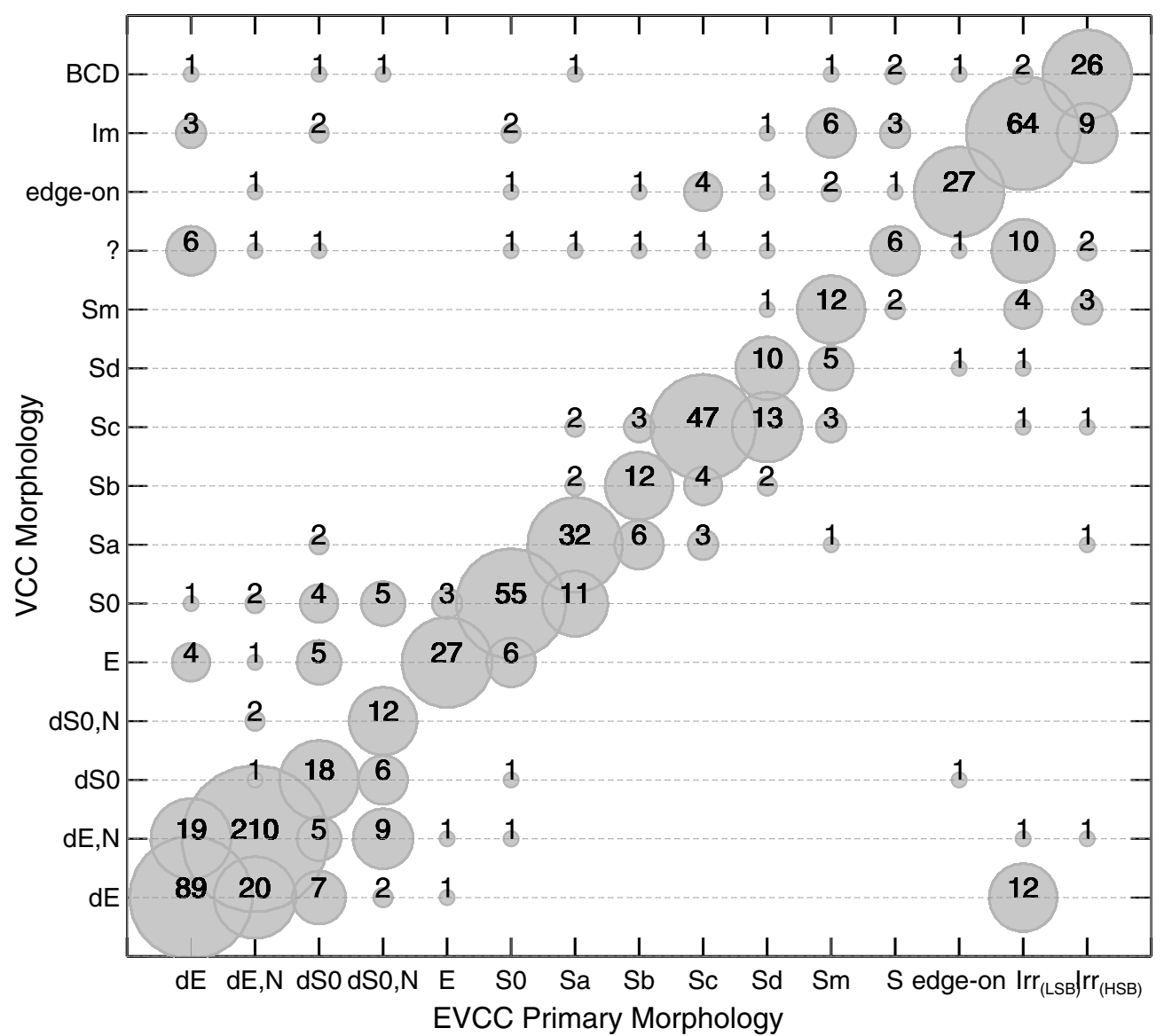

Figure 15. Comparison between EVCC primary morphological classification and VCC classification for 913 objects in common. The symbol size is proportional to the number of galaxies.

irregulars (see the lower right part of Figure 15) in the EVCC. In our classification, while some of these galaxies present elliptical shapes in outer part, they show large irregular structures with blue colors at their centers (e.g, EVCC 187, EVCC 246, EVCC 301, EVCC 316, EVCC 374, EVCC 400, EVCC 1143, and EVCC 2227 in Figure 17). Besides, some other galaxies show significantly off-center blue cores (e.g., EVCC 159, EVCC 245, EVCC 653, and EVCC 960 in Figure 17).

2. Combining properties like angular size and surface brightness distribution led to the reclassification of some earlytype galaxies (E and S0) in the VCC to early-type dwarf galaxies (dE and dS0) in the $\mathrm{EVCC}^{8}$ (see Figure 18). While the main criterion for distinguishing $\mathrm{dE} / \mathrm{dS} 0$ from $\mathrm{E} / \mathrm{S} 0$ has been the faint surface brightness of the former, this distinction becomes increasingly difficult at faint $\left(M_{r}>\right.$ -18 ) magnitudes. At these magnitudes, changes from $\mathrm{E} / \mathrm{S} 0$ to $\mathrm{dE} / \mathrm{dS} 0$ are therefore expected between two surveys with different instruments and especially different bands, namely, the blue-sensitive VCC and the multi-band EVCC. We could not see that these galaxies have a particu-

\footnotetext{
8 While our morphological classification of $\mathrm{E} / \mathrm{S} 0 / \mathrm{dE} / \mathrm{dS} 0$ was made by visual inspection of images, based on the peak surface brightness values of galaxies returned from SExtractor, we found that the peak surface brightness limit for discrimination between $\mathrm{E} / \mathrm{S} 0$ and $\mathrm{dE} / \mathrm{dS} 0$ is $\mu_{r} \sim 19 \mathrm{mag} \operatorname{arcsec}^{-2}$. In the $\mathrm{R}(50)$ versus peak surface brightness diagram, the size of $\mathrm{E} / \mathrm{S} 0$ galaxies decreases with peak surface brightness in a wide range of $0.5<\log (\mathrm{R} 50)<$ 2.0 , whereas $\mathrm{dE} / \mathrm{dS} 0$ galaxies have almost a constant size in a relatively narrow range of $0.5<\log (\mathrm{R} 50)<1.5$.
}

larly high surface brightness or are particularly compact in our classification.

3. The dwarf lenticular galaxies $(\mathrm{dSO}$ and $\mathrm{dSO}(\mathrm{N}))$ in the EVCC have large scatter in the VCC morphological classification: about 55\% (44 of 80) of dwarf lenticular galaxies in the EVCC were classified as different types in the VCC. A significant fraction of these galaxies are classified as $\mathrm{E}$ and $\mathrm{dE}$ types in the VCC, however, they show prominent irregularities at the center and/or asymmetric features like bar or lens in SDSS images (see Figure 19).

4. Some ( 20 galaxies) dEs in the VCC have been re-classified as $\mathrm{dE}(\mathrm{N})$ in the EVCC. As shown in Figure 20 (top panels), from SDSS images of dEs in the VCC, clear nuclei are visible. It is thought that a fraction of dEs were missclassified in the VCC because either the resolution of the photographic plate material or the seeing was insufficient to detect a central point source. Furthermore, three dSOs in the VCC also have been assigned to the $\mathrm{dSO}(\mathrm{N}) \mathrm{s}$ in the EVCC (see the middle panels of Figure 20). On the other hand, some (19 galaxies) $\mathrm{dE}(\mathrm{N}) \mathrm{s}$ in the VCC do not show nuclei at their centers from SDSS images and they have been re-classified as normal dEs in the EVCC (see bottom panels of Figure 20).

\subsection{Projected Spatial Distribution of Galaxies}

Figure 21(top, left panel) shows the distribution of galaxies on the sky classified as certain members (red circles) and possible members (blue circles) in the EVCC. For comparison, we also present the distribution of VCC galaxies (see the right 

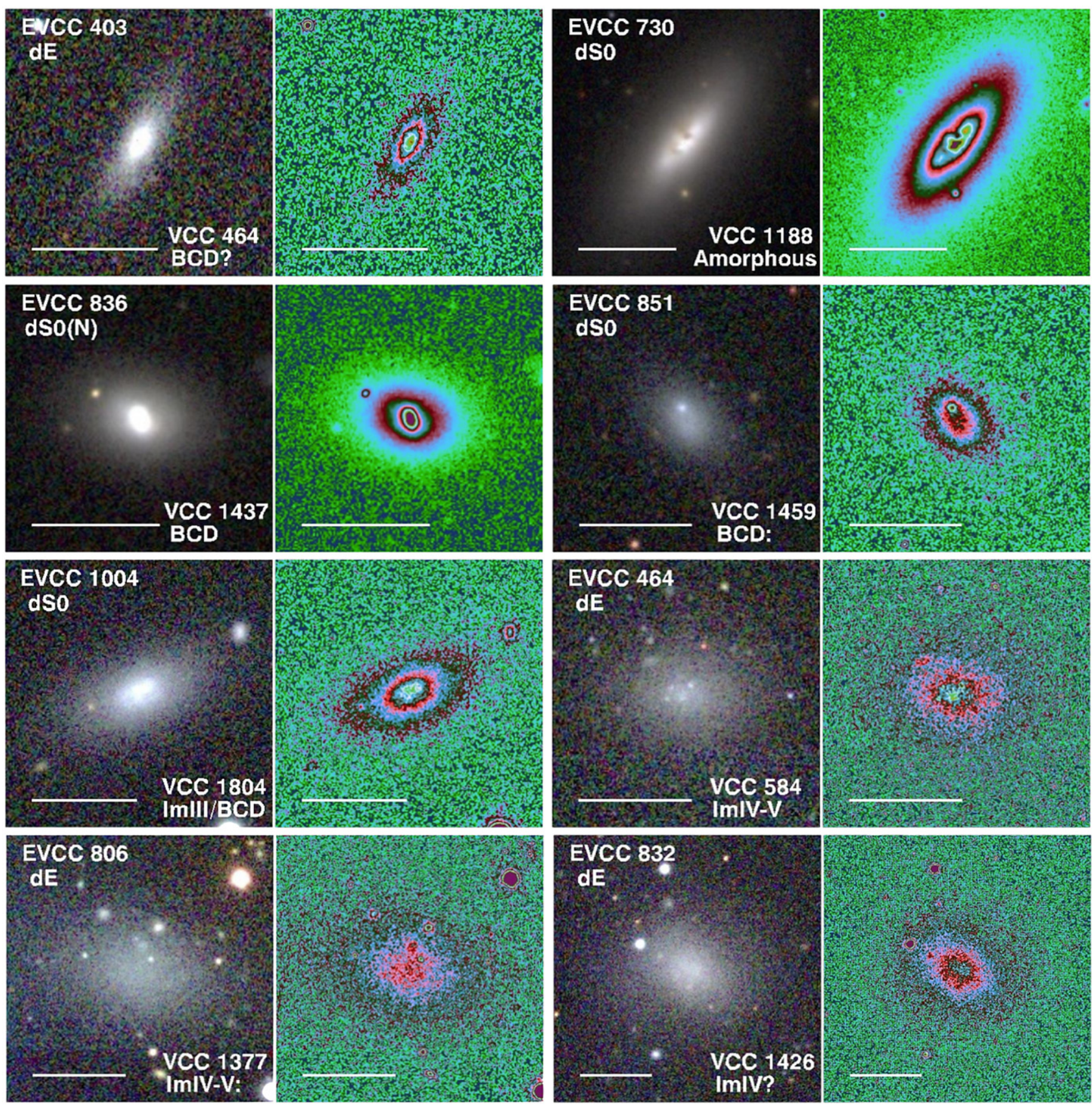

Figure 16. Examples of eight VCC galaxies with late-type morphology that were reclassified as early-type dwarf galaxies in the EVCC. The left and right panels show the SDSS gri combined color and $r$-band images, respectively. The intensity is represented by alternating colors in the $r$-band image to highlight the shape of isophotes and substructures within a galaxy. The solid bar corresponds to 30 arcsec on the sky. North is up and east is to the left.

(A color version of this figure is available in the online journal.)

panel of Figure 21(top)). Member galaxies in the EVCC are widely distributed beyond the region of the VCC. Basically, the distributions of certain and possible member galaxies in the EVCC appear to be similar to those in the VCC within the area of the VCC. As previous studies suggested (Sandage et al. 1985b; Binggeli et al. 1987, 1993), subgroups (W and M) of the Virgo cluster are dominated by possible members, and this feature is also seen in the EVCC. On the other hand, the majority of EVCC galaxies in the $\mathrm{W}^{\prime}$ group are classified as certain members in contrast to the case of the VCC. All of galaxies in the southern extension area (decl. $<5 \mathrm{deg}$ ) are defined as possible members in the VCC (see also Figure 1 and 2 of Sandage et al. 1985b). However, in the case of the EVCC, a large fraction of certain member galaxies are mixed with possible members in this region. Furthermore, a significant fraction of certain member galaxies in the EVCC are also found in the eastern part of the southern extension of the Virgo cluster, which overlaps with previously known galaxy groups (e.g., NGC 4636, Brough et al. 2006).

Most galaxies in the $\mathrm{W}$ and $\mathrm{M}$ clouds are defined as possible members in the VCC since they are at their mean velocity of $\sim 2000 \mathrm{~km} \mathrm{~s}^{-1}$. They are thought to lie at about twice the distance 

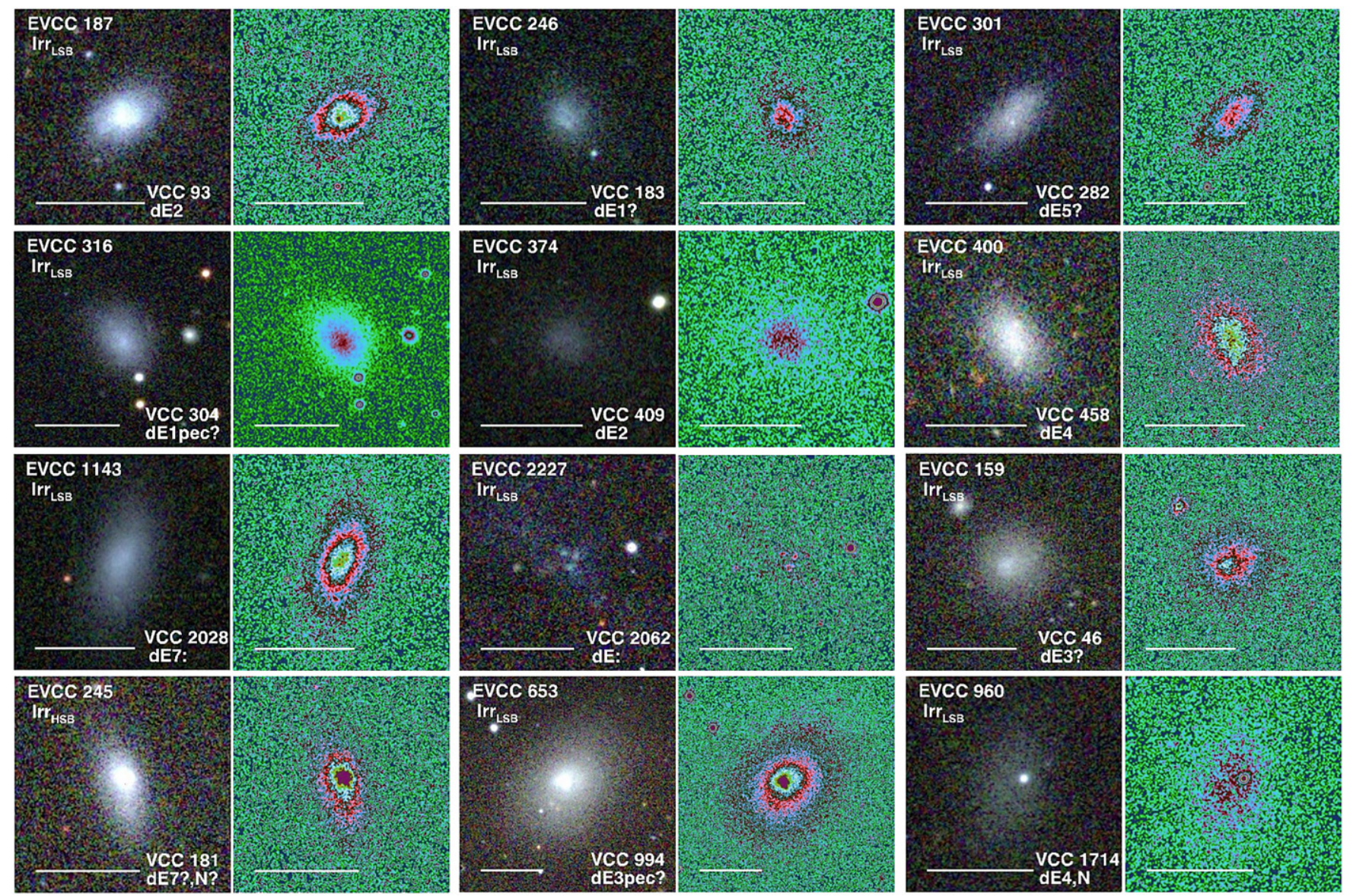

Figure 17. Examples of $12 \mathrm{VCC}$ early-type dwarf galaxies that were reclassified to irregular galaxies in the EVCC. The left and right panels show the SDSS gri combined color and $r$-band images, respectively. The intensity is represented by alternating colors in the $r$-band image to highlight the shape of isophotes and substructures within a galaxy. The solid bar corresponds to 30 arcsec on the sky. North is up and east is to the left.

(A color version of this figure is available in the online journal.)

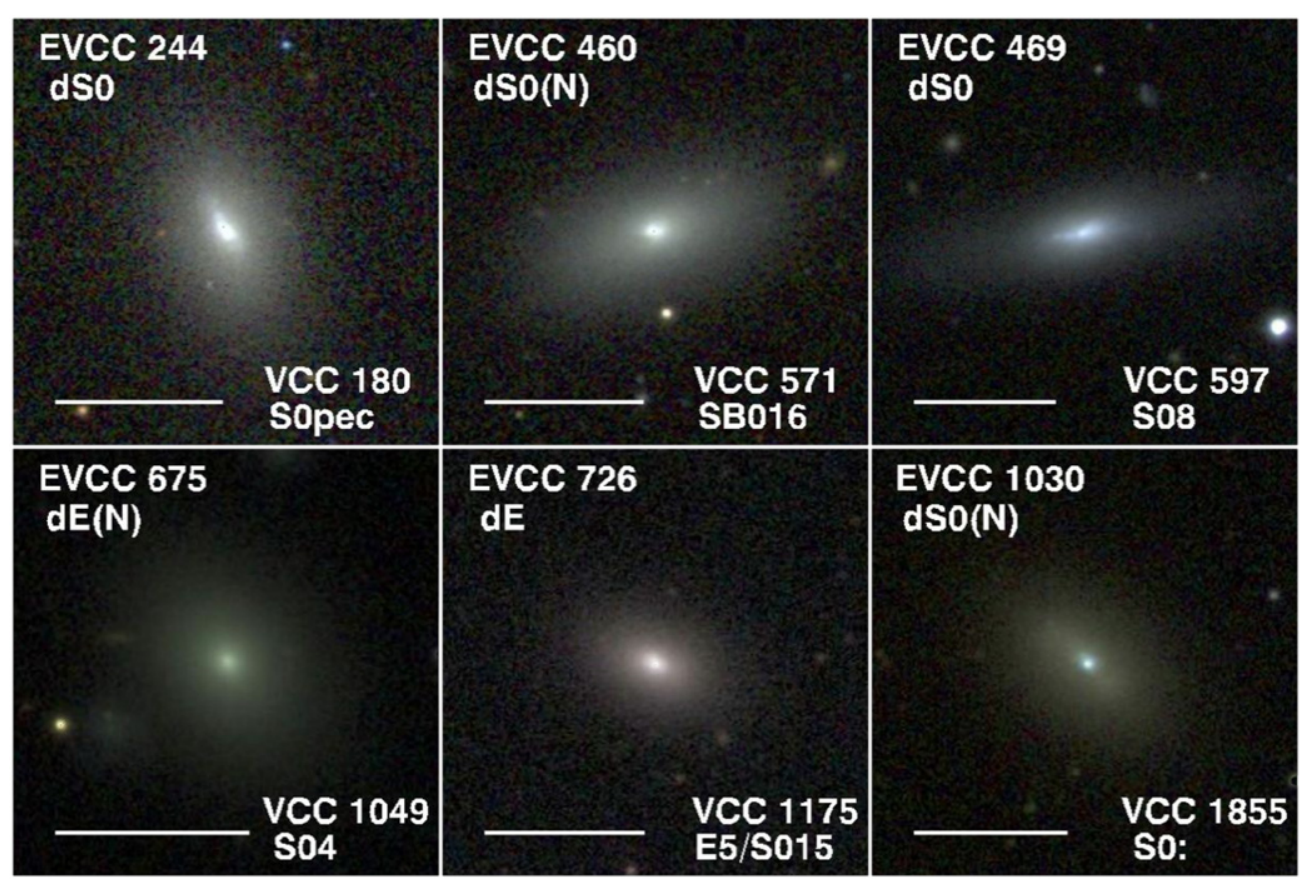

Figure 18. Examples of reclassification to $\mathrm{dE} / \mathrm{dS} 0$ galaxies in the EVCC from E/S0 morphologies in the VCC. The solid bar corresponds to 30 arcsec on the sky. North is up and east is to the left.

(A color version of this figure is available in the online journal.) 


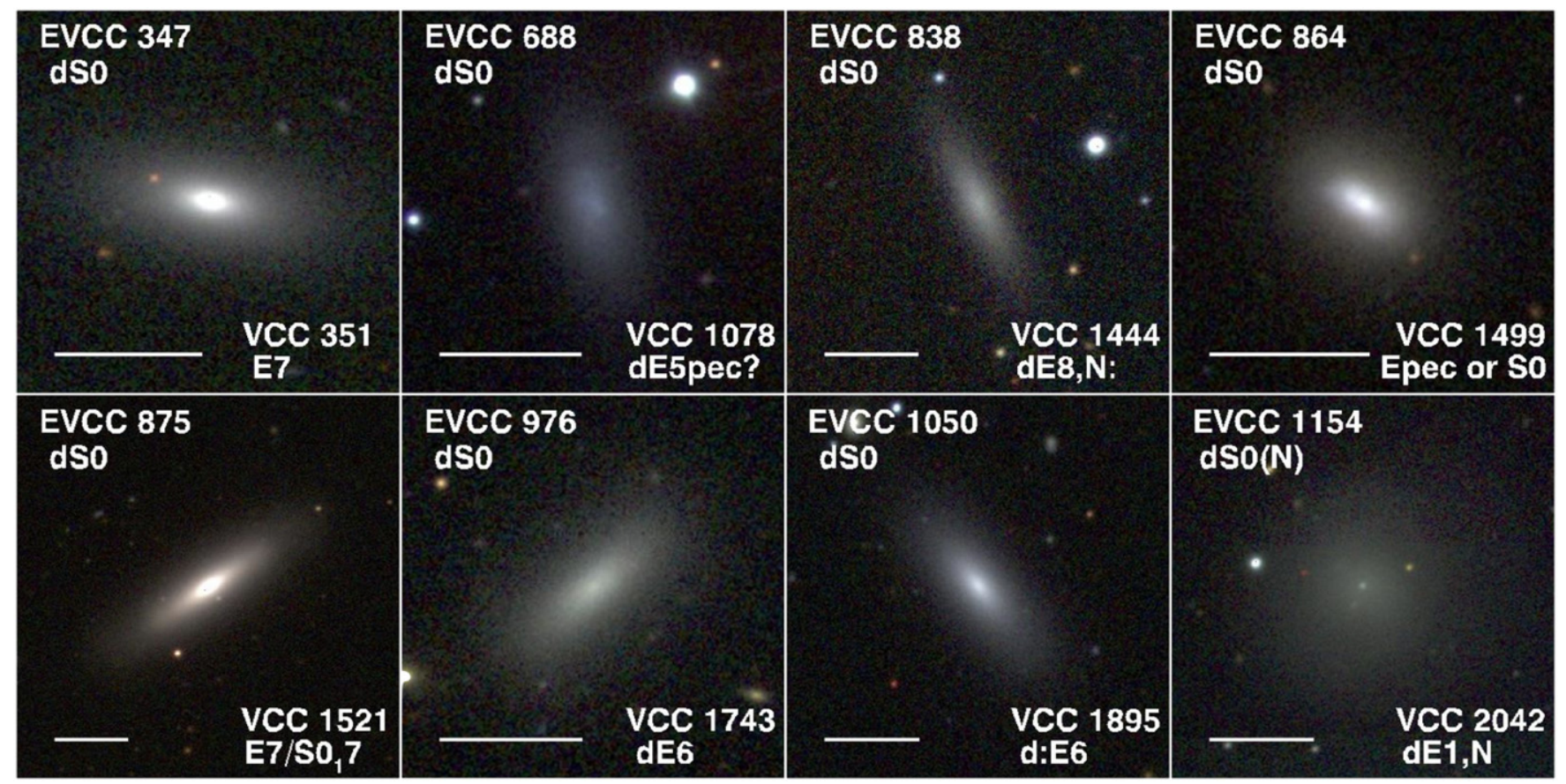

Figure 19. Examples of eight dwarf lenticular (dS0) galaxies in the EVCC with different morphology in the VCC. The solid bar corresponds to 30 arcsec on the sky. North is up and east is to the left.

(A color version of this figure is available in the online journal.)

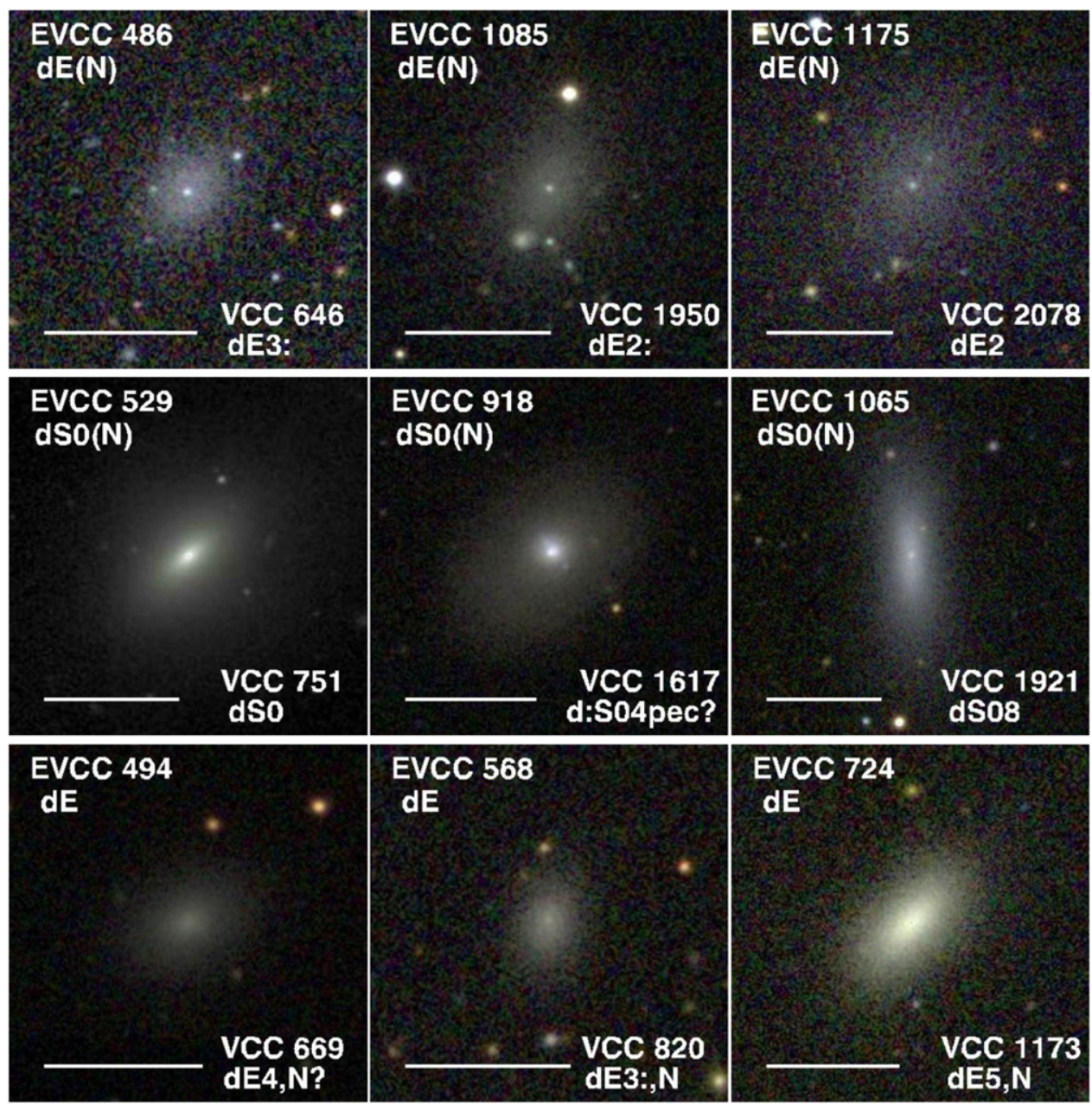

Figure 20. Top and middle panels: examples of SDSS images of dEs and dS0s where we found a nucleus. Bottom panels: examples of SDSS images of three dEs where a nucleus could not be confirmed. The solid bar corresponds to 30 arcsec on the sky. North is up and east is to the left. 

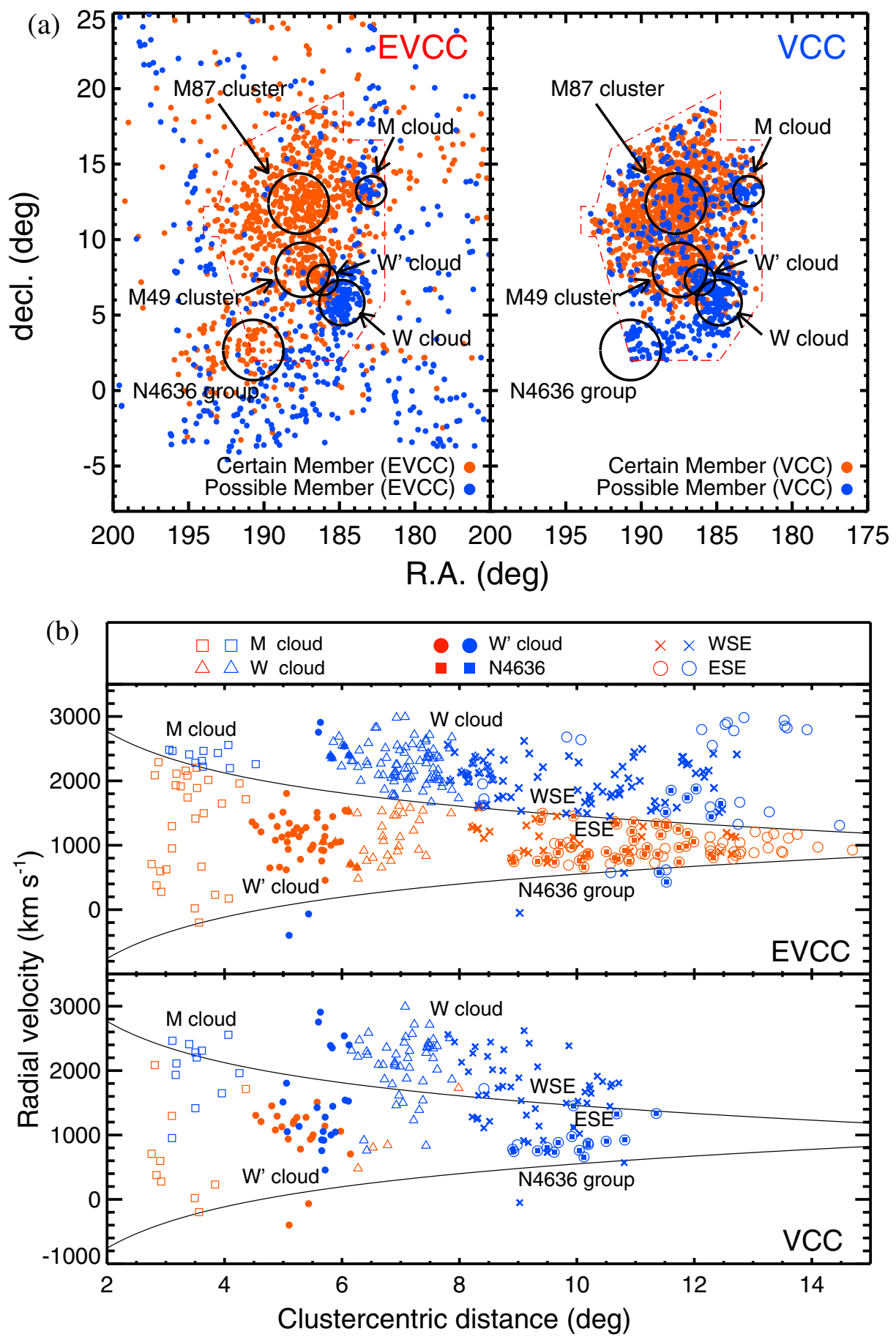

Figure 21. Top: projected spatial distribution of galaxies in the EVCC (left) and VCC (right). Red and blue filled circles are certain and possible member galaxies, respectively. The membership information of the right panel is from the VCC. The red dot-dashed contour outlines the survey boundary of the VCC. The subgroups (M87, M49, M, W, and $\mathrm{W}^{\prime}$ ) are presented by large circles as defined by Binggeli et al. (1987) and the NGC 4636 group is defined by this study. Note that the size of the large circle does not represent the physical extend of each subgroup. Bottom: same as Figure 4, but for galaxies in the EVCC and VCC which are associated with well-known subgroups in the Virgo cluster. Galaxies included in different subgroups are denoted as different symbols; open squares for M cloud, filled circles for W' cloud, open triangles for W, open circles for eastern part of the southern extension (ESE), crosses for western part of the southern extension (WSE), and filled squares for NGC 4636 group. The ESE and WSE are defined as $189 \mathrm{deg}<$ R.A. $<197 \mathrm{deg}, 0 \mathrm{deg}<\mathrm{decl} .<5 \mathrm{deg}$ and $184 \mathrm{deg}<\mathrm{R}$.A. $<189 \mathrm{deg}, 0$ deg $<$ decl. $<5$ deg, respectively. In each panel, red and blue symbols represent the certain and possible member galaxies defined from each catalog, respectively.

(A color version of this figure is available in the online journal.)

of the Virgo cluster (Binggeli et al. 1993). These clouds might be linked to each other (Ftaclas et al. 1984; Yoon et al. 2012) and are falling into the Virgo cluster from the backside (Yasuda et al. 1997). However, Gavazzi et al. (1999) argue that the W cloud is a rather isolated structure following the Hubble flow, whereas the M cloud appears to fall into the cluster. In the EVCC, the $\mathrm{W}$ cloud (open triangles in the upper panel of Figure 21 (bottom)) appears to be a localized, independent structure in 
which a large fraction of galaxies reside outside of the caustic curves of the infall model lines. On the other hand, the majority of galaxies in the M cloud turn out to be certain members which are inside of the infall models (open squares in the upper panel of Figure 21 (bottom)). In this case, we suggest that the $\mathrm{M}$ cloud consists of a galaxy population infalling into the Virgo cluster and spatially projected galaxies associated with main body of the cluster.

Binggeli et al. (1987) proposed that the $\mathrm{W}^{\prime}$ cloud might be in between the $\mathrm{W}$ cloud and cluster $\mathrm{B}$. It has been further suggested that the $\mathrm{W}^{\prime}$ cloud is connected to the background $\mathrm{W}$ cloud as a filament and both are falling into cluster B (Binggeli et al. 1993). However, Mei et al. (2007) proposed that the $\mathrm{W}^{\prime}$ could be a more localized structure behind the Virgo cluster based on the distance estimation of five galaxies $(\sim 22.9 \pm 0.3 \mathrm{Mpc}$ in the mean). In the EVCC, most galaxies associated with the $\mathrm{W}^{\prime}$ cloud are well inside of the infall model lines and have a mean velocity $\left(\sim 1140 \mathrm{~km} \mathrm{~s}^{-1}\right)$ comparable to the systemic Virgo cluster velocity (filled circles in the upper panel of Figure 21(bottom)). Consequently, considering its estimated distance (Mei et al. 2007) combined with our velocity distribution, we suggest that galaxies in the $\mathrm{W}^{\prime}$ cloud are falling into the Virgo cluster from behind.

To the south of the Virgo cluster (i.e., decl. $<5 \mathrm{deg}$ ), the southern extension defines an extended structure which seems to be a filament in the Local Supercluster (de Vaucouleurs \& de Vaucouleurs 1973; Tully 1982; Hoffman et al. 1995). The southern extension is also suggested as an infalling structure to the Virgo cluster (e.g., Binggeli et al. 1987). Furthermore, the western part of this structure might be linked to the $\mathrm{W}$ and $\mathrm{M}$ clouds (Yoon et al. 2012). In the VCC, galaxies in the southern extension were classified as either possible members or as background objects. However, in the EVCC, some galaxies in the southern extension are classified as certain members. Note that such certain members are preferentially located on the eastern side of the southern extension (hereafter ESE), while the western side (hereafter WSE) is dominated by possible members (see also the left panel of Figure 21(top)). While the galaxies associated with the ESE are far from the Virgo center, most of them are well inside of the narrow range of the infall model lines. The ESE is mostly associated with the NGC 4636 group which exhibits intragroup X-ray emission and is dynamically mature (Brough et al. 2006). Previous studies suggest that the NGC 4636 group is also infalling into the Vigo cluster (Tully \& Shaya 1984; Nolthenius 1993).

In Figure 22, we present the projected spatial distribution of galaxies in the EVCC (middle and bottom panels) in comparison with those in the VCC (top panels) according to the morphology of galaxies (left and right panels for early- and late-type galaxies, respectively). The spatial distribution of E/S0 galaxies in the EVCC appear to be similar to that of the VCC. This is supported by a Kolmogorov-Smirnov (K-S) statistic that is equivalent to $99 \%$. This is expected as the morphological classifications of most early-type galaxies between the EVCC and VCC agree (see Section 6.1). Early-type galaxies are strongly concentrated around M87 and the centers of subgroups: about $60 \%$ of early-type and $30 \%$ of late-type galaxies are located within an angular distance of $2 \mathrm{deg}$ (corresponding to $575 \mathrm{kpc}$ ) from M87 and subgroups. Furthermore, they appear to be surrounded by early-type dwarf galaxies (dEs and dS0s): about $80 \%$ of $\mathrm{dE} / \mathrm{dSO}$ galaxies reside within an angular distance of $1 \mathrm{deg}$ (corresponding to $288 \mathrm{kpc}$ ) from E/S0 galaxies. One interesting feature is that the EVCC reveals a population of E/S0 galaxies around and south of the NGC 4636 group, a region that was not covered by the VCC. It is clear that early-type and late-type galaxies in the EVCC are dominated by RA/RE- and BA/BEtype galaxies, respectively (see the bottom panels of Figure 22): a K-S test gives that the spatial distributions of RA/RE- and BA/ BE-type galaxies are similar to those of early-type and late-type galaxies at the $97 \%$ and $95 \%$ confidence level, respectively. The early-type galaxies with red spectra (RA and RE types) seem to concentrate around the main body and subgroups of the Virgo cluster, whereas those with BA and BE types are preferentially located in their outskirts: about $85 \%$ of early-type galaxies residing within the main body and subgroups are those of RA and RE types.

Late-type galaxies (spirals and irregulars) are relatively more scattered and randomly distributed compared to the early-type galaxies. Furthermore, the region outside of the VCC survey area, which is covered only by the EVCC, is dominated by latetype galaxies. Most of the subgroups ( $\mathrm{W}, \mathrm{W}^{\prime}$, and $\mathrm{M}$ clouds) contain many late-type galaxies, whereas the NGC 4636 group lacks these galaxies.

\subsection{Luminosity Function}

Figure 23 shows the $r$-band galaxy luminosity function in the EVCC in comparison with that of the VCC. The luminosity function of EVCC galaxies does not include galaxies fainter than $r \approx 19$, which is mainly due to the limiting magnitude of SDSS spectroscopic observations (i.e., $r=17.7$, vertical dashed line). Furthermore, the apparent incompleteness of the luminosity function is also seen at a brighter magnitude $(r \sim 16.5)$ than the nominal spectroscopic limit, which is consistent with the decrease of the $\mathrm{S} / \mathrm{P}$ ratio in Figure 3. As expected from the type-specific galaxy luminosity functions (Binggeli et al. 1988; Ferguson \& Sandage 1988; Jerjen \& Tammann 1997), these faint samples in the VCC are found to be $\mathrm{dE}$ and Irr galaxies (see also Figures 24 and 25). The mean magnitude of galaxies that are exclusive to the EVCC (dark gray histogram) is $\sim 1.6 \mathrm{mag}$ fainter than that of galaxies that are in common with the VCC (light gray histogram). This indicates that the EVCC galaxies located outside of the VCC region have systematically lower luminosities. This is a consequence of the morphology-density relation where the low-density environments lack the luminous early-type galaxies found in the inner regions of galaxy clusters.

In Figures 24 and 25, we present the galaxy luminosity function in the EVCC (left panels) and VCC (right panels) for various galaxy morphological types. In each panel, the hatched histogram is for galaxies included both in the EVCC and VCC. First, for a given galaxy morphological type, the shape of the luminosity function of the EVCC appear to be comparable to that of the VCC in the sense of similar magnitude range and mean magnitude of the distribution. It is shown in Figure 25 that the mean magnitude of the distribution changes with galaxy type. In particular, the mean magnitude of late-type galaxies systematically varies in the sense that the Irr and Sm galaxies are fainter than spiral galaxies and the $\mathrm{Sd}$ galaxies are fainter than the Sa, Sb, and Sc galaxies (Sandage et al. 1985b; Binggeli et al. 1988; Ferguson \& Sandage 1988; Jerjen \& Tammann 1997).

In the case of galaxies in the VCC (right panels of Figures 24 and 25), most galaxies are recovered by the EVCC. However, about $67 \%$ and $42 \%$ of $\mathrm{dE}$ and Irr galaxies in the VCC, respectively, are not included in the EVCC. A considerable 


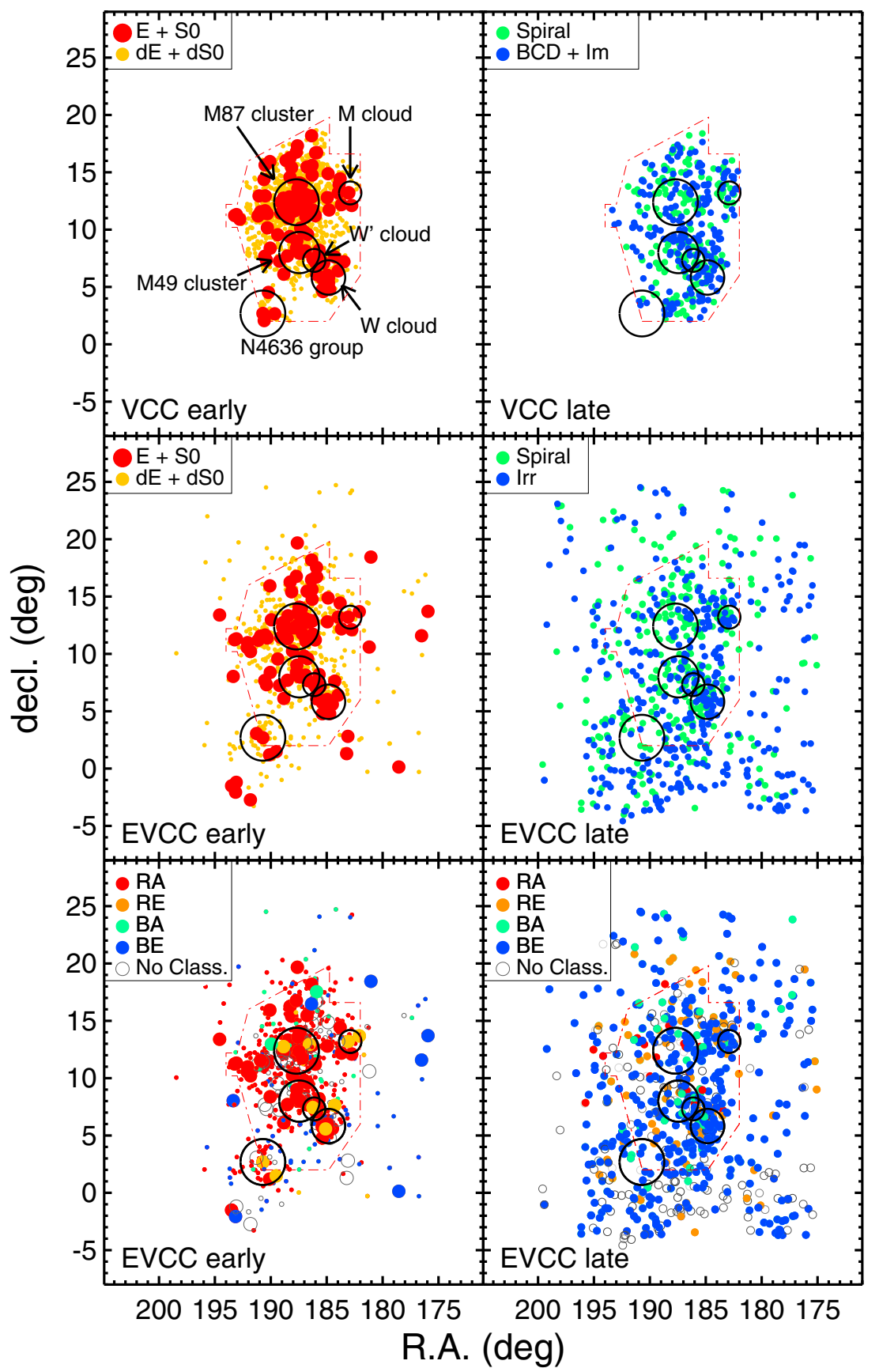

Figure 22. Comparison of the projected spatial distributions of galaxies between the VCC (top panels) and EVCC (middle and bottom panels) according to the galaxy morphology. Left and right panels are for early- $(\mathrm{E} / \mathrm{S} 0$ and $\mathrm{dE} / \mathrm{dS} 0)$ and late-type (spirals and irregulars) galaxies, respectively. In the bottom panels, filled circles with different colors represent different secondary morphologies in the EVCC, and open circles are galaxies with no available secondary classification information which are only compiled from the NED and have no information of SED.

(A color version of this figure is available in the online journal.)

fraction of these galaxies are fainter than the limiting magnitude of the SDSS spectroscopic survey. On the other hand, some dE and Irr galaxies brighter than this magnitude limit are also not found in the EVCC. While these galaxies are certain and possible members in the VCC, they are excluded in the EVCC owing to their larger radial velocities (i.e., $>3000 \mathrm{~km} \mathrm{~s}^{-1}$ ). In the case of galaxies in the EVCC (left panels of Figures 24 and 25), at all magnitudes, some EVCC galaxies are not found in the VCC. This is because most of them are located outside of the VCC region. This feature is more distinct for the Sm- and Irr-type galaxies, since these galaxies are preferentially found in the outskirts of clusters.

\subsection{Astrometry and Membership of VCC Galaxies}

By matching the coordinates of all galaxies presented in the VCC with those from SDSS images, we have found that coordinates of large numbers of galaxies in the VCC significantly deviate from our coordinates and are discrepant from the true centers of galaxies. Moreover, a considerable fraction of galaxies in NED also show incorrect coordinates. For VCC galaxies with incorrect coordinates, by taking into account of their size of D25, magnitude, and morphology, we identify real objects from visual inspection of SDSS images. As a result, about $28 \%$ of galaxies in the VCC (586 of 2096) turned 


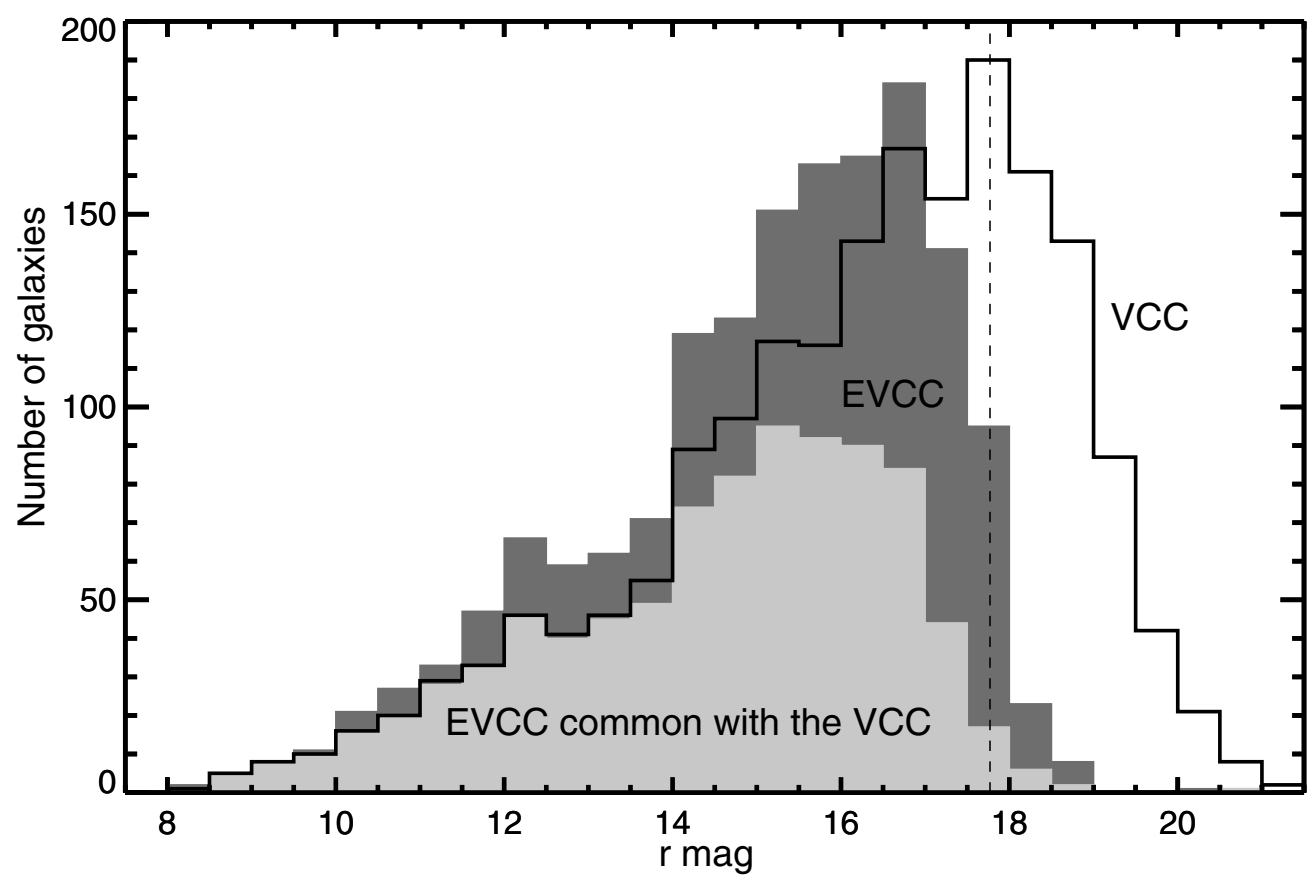

Figure 23. Galaxy luminosity function in the EVCC (dark gray histogram) in comparison with that of the VCC (open histogram). The open histogram is for all certain and possible member galaxies in the VCC. The light gray histogram is for the EVCC galaxies that are in common with the VCC. The vertical dashed line is the limiting magnitude of the SDSS spectroscopic survey (i.e., $r=17.7$ ).

out to have different coordinates from the EVCC with offsets larger than $10 \operatorname{arcsec}$ (see Figure 26 for residuals of coordinates between the EVCC and VCC). In our EVCC (see Tables 2 and 3), coordinates of all galaxies in the VCC and NED have been superseded by those returned from our own photometry.

The cluster membership of galaxies in the VCC was solely based on morphology, confirmed by the velocity data available for some galaxies (Binggeli et al. 1985). In Table 4, we compare the membership of VCC galaxies with radial velocity information in the EVCC (see also Figure 27). We divide galaxies into those with larger or lower velocities than the velocity limit of $3000 \mathrm{~km} \mathrm{~s}^{-1}$. First, only about $12 \%(84 / 715)$ of certain member galaxies in the VCC are found to have larger radial velocities than this velocity limit. In the case of VCC possible members, about $27 \%(96 / 353)$ of galaxies turn out to have radial velocities larger than $3000 \mathrm{~km} \mathrm{~s}^{-1}$. On the other hand, about $10 \%(25 / 246)$ of background objects in the VCC are within this limit of radial velocity. Binggeli et al. (1993) estimated that based on the velocity information available for 144 galaxies, the success rate for the morphology-based cluster membership of the VCC is $95 \%$. However, when we carefully examined Table 1 of Binggeli et al. (1993) in detail, we found that the change of membership status is slightly high compared to the result presented in Table 4: $\sim 13 \%$ (6 of 45) from certain member to background, $\sim 33 \%$ ( 31 of 94 ) from possible member to background, and $\sim 20 \%$ ( 1 of 5) from background to certain member.

In Figure 28, we present the projected spatial distribution of 676 galaxies that are exclusive to the EVCC. While most of these galaxies are distributed outside of the VCC region, about $19 \%$ of galaxies are still located inside of the VCC region (see also Davies et al. 2014). About 51\% and $49 \%$ of the latter are certain and possible member galaxies, respectively. It is interesting to note that these new galaxies typically have magnitudes fainter than $r>15$ and show morphologies of Irr and $\mathrm{dE} / \mathrm{dS} 0$. Furthermore, these galaxies show systematically smaller effective radii at a fixed magnitude than VCC galaxies (see Figure 29). This indicates that these galaxies are more compact with centrally concentrated light distribution when compared to the majority of galaxies in the VCC. We therefore suspect that the comparatively poor angular resolution of the VCC photographic plates prevented a morphology-based membership assignment of these galaxies in the VCC. In addition, the fact that these more compact galaxies bare a resemblance to background galaxies could be the reason why they were rejected as cluster members in the VCC study.

\section{SUMMARY AND CONCLUSIONS}

We have presented a new catalog of galaxies in the Virgo cluster and wider area, the Extended Virgo Cluster Catalog (EVCC), based on the SDSS DR7 and NED data. The EVCC covers a rectangular region $\left(60.1 \mathrm{Mpc}^{2}\right.$ assuming a distance of the Virgo cluster of $16.5 \mathrm{Mpc}$ ), which is 5.2 times larger than the footprint of the classical VCC. This paper focuses on a broad overview of the construction of the EVCC including selection of sample galaxies as well as their morphological classification and measurements of photometric parameters. The main results of this paper are summarized as follows.

1. We focus on a sample galaxies with radial velocities that are available in the SDSS and NED spectroscopic data, taking advantage of radial velocity as a suitable information for cluster membership for galaxies in a galaxy cluster. We thus selected all galaxies in the EVCC region with radial velocities less than $3000 \mathrm{~km} \mathrm{~s}^{-1}$ as a means to separate member galaxies from background. This process secured a total of 1589 galaxies, 676 of which are not part of the VCC. The member galaxies are divided into two subsamples using a spherical symmetric infall model in a plot of radial velocity versus clustercentric distance of galaxies. Certain and possible Virgo cluster members are defined as those located inside and outside of the model lines, respectively. 


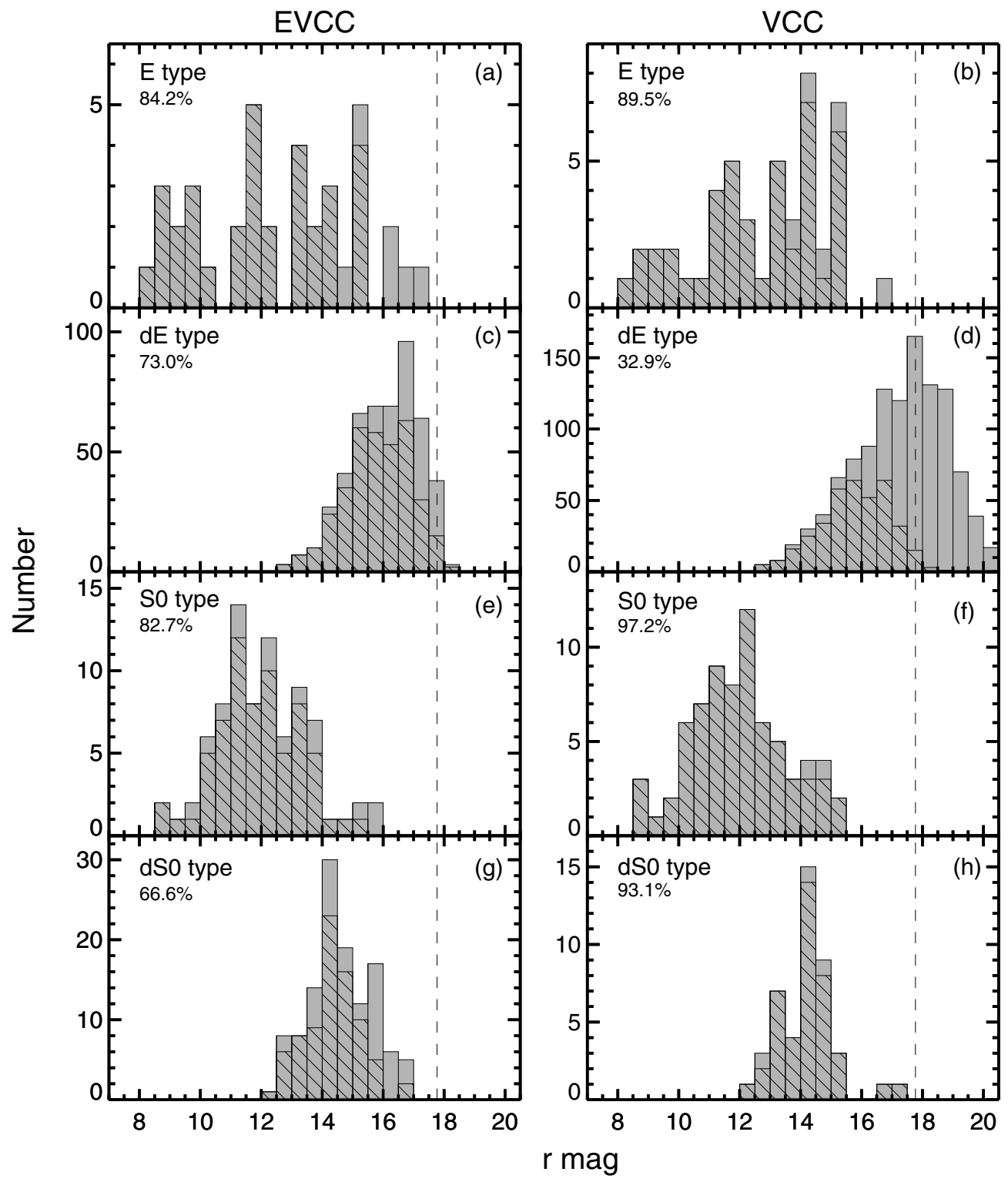

Figure 24. Galaxy luminosity function of early-type galaxies (E, dE, S0, and dS0) in the EVCC (left panels) and VCC (right panels). Certain and possible member galaxies are considered. In each panel, the hatched histogram is for galaxies included both in the EVCC and the VCC, and its fraction is also indicated below the galaxy morphological type.

Table 4

VCC Galaxies with Redshift Data

\begin{tabular}{lcccr}
\hline \hline & VCC Certain Member & VCC Possible Member & VCC Background & Total \\
\hline$c z<3000 \mathrm{~km} \mathrm{~s}^{-1}$ & 631 & 257 & 25 & 913 \\
$c z>3000 \mathrm{~km} \mathrm{~s}^{-1}$ & 84 & 96 & 221 & 401 \\
\hline Total & 715 & 353 & 246 & 1314 \\
\hline
\end{tabular}

2. We introduced two independent, complementary morphological classification schemes using the SDSS imaging and spectroscopic data: the primary morphology and secondary morphology. The primary morphology is based on the extended Hubble morphological classification scheme used in the VCC. The secondary morphological classification is using the SED shape and the presence of $\mathrm{H} \alpha$ emission/ absorption lines. The EVCC galaxies were subdivided into 21 classes in the primary and four classes in the secondary morphology.

3. The primary and secondary morphologies show an expected general trend in the sense that early-type galaxies exhibit red spectra and the majority of late-type galaxies show blue spectra, although some scatter in the main trend between two morphologies are shown. This correlation implies that the secondary morphology, which is based on the spectrum of the central region of galaxy, also reflects the global characteristics of galaxies. Furthermore, we found that within our classification scheme, fiber location in the SDSS does not significantly affect the secondary morphology at a given primary morphology.

4. The final EVCC catalog includes fundamental information of galaxies such as membership, morphology, and photometric parameters. In addition, we also computed 


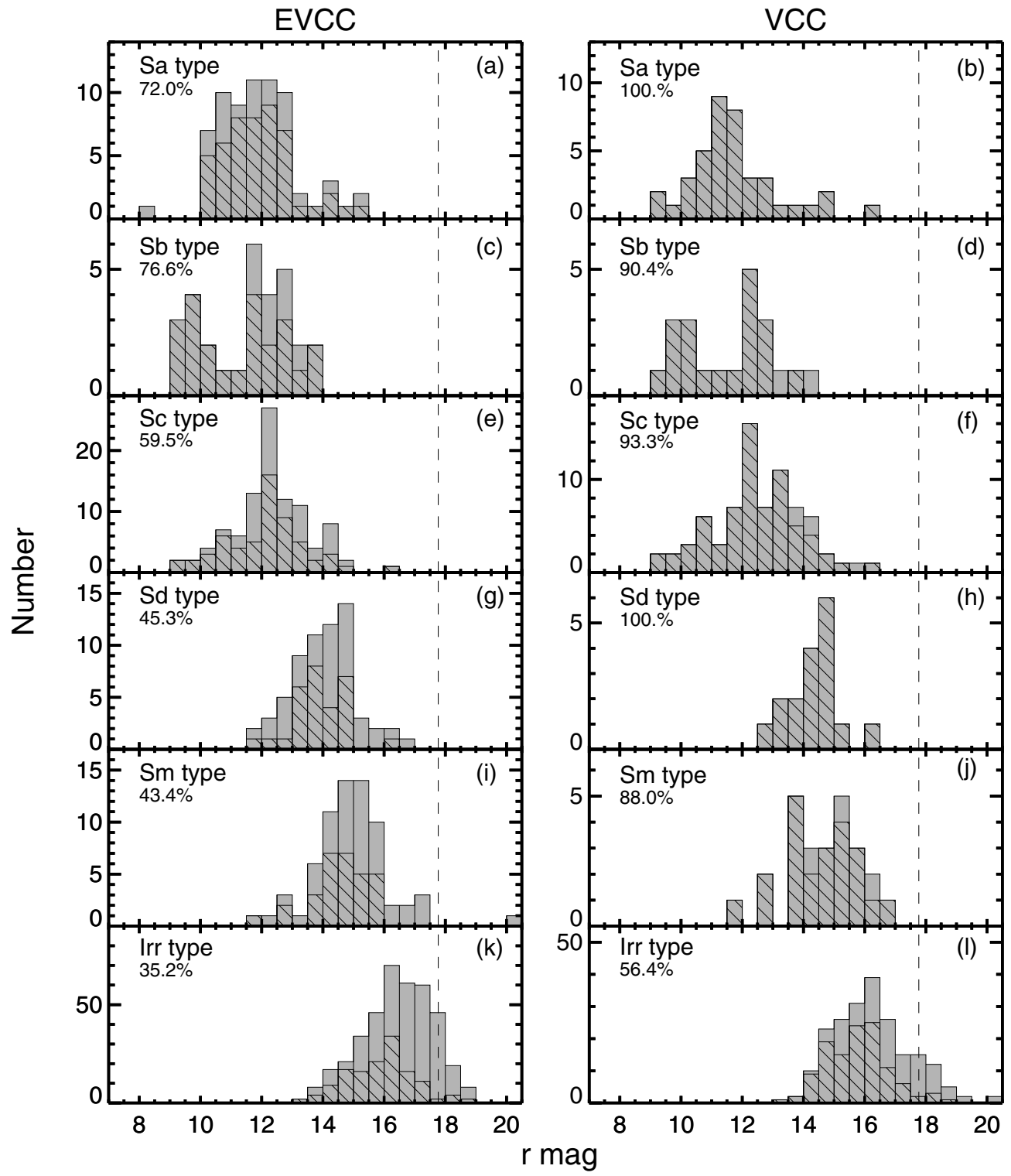

Figure 25. Same as Figure 24, but for late-type galaxies (Sa, Sb, Sc, Sd, Sm, and Irr) in the EVCC (left panels) and VCC (right panels).

photometric parameters for 1183 galaxies in the VCC that are not in common with the EVCC. The photometry of all galaxies was performed using our own SExtractor pipeline in order to remedy deficiencies in the SDSS photometry caused by deblending and sky subtraction.

5. The primary morphology in the EVCC is generally consistent with the VCC classification in which a close trend along the diagonal between two catalogs is found. The relationship has some outliers at a given morphological type where the morphologies of galaxies in our EVCC disagree with those in the VCC. However, it is worth noting that, in most cases, the morphologies of these galaxies have been updated in the EVCC thanks to the SDSS images with higher resolution and $\mathrm{S} / \mathrm{N}$ ratio compared to the photographic plate images of the VCC.

6. Member galaxies in the EVCC are widely distributed beyond the region of the VCC. Basically, the certain and possible member galaxies in the EVCC show the projected spatial distributions similar to counterparts in the VCC within the area of the VCC. The W subgroup of the Virgo cluster is dominated by possible members in the EVCC. On the other hand, the majority of EVCC galaxies in the $\mathrm{W}^{\prime}$ subgroup turned out to be certain members in contrast to the case of the VCC. A large fraction of certain member galaxies in the EVCC are found in the southern extension, while all of VCC galaxies in this region are defined as possible members. In particular, a significant fraction of certain member galaxies in the EVCC are distributed toward the eastern part of the southern extension including the previously known galaxy group of NGC 4636.

7. The overall spatial distribution of EVCC galaxies according to morphology is similar to that of the VCC. The earlytype galaxies are strongly concentrated around M87 and the centers of subgroups, whereas late-type galaxies are more evenly distributed. Outside of the VCC area, the galaxy populations are dominated by late-type galaxies. It is worth noting that the EVCC reveals many early-type galaxies in the eastern part of the southern extension (i.e., around and below the NGC 4636 group), a region that was not covered by the VCC. 


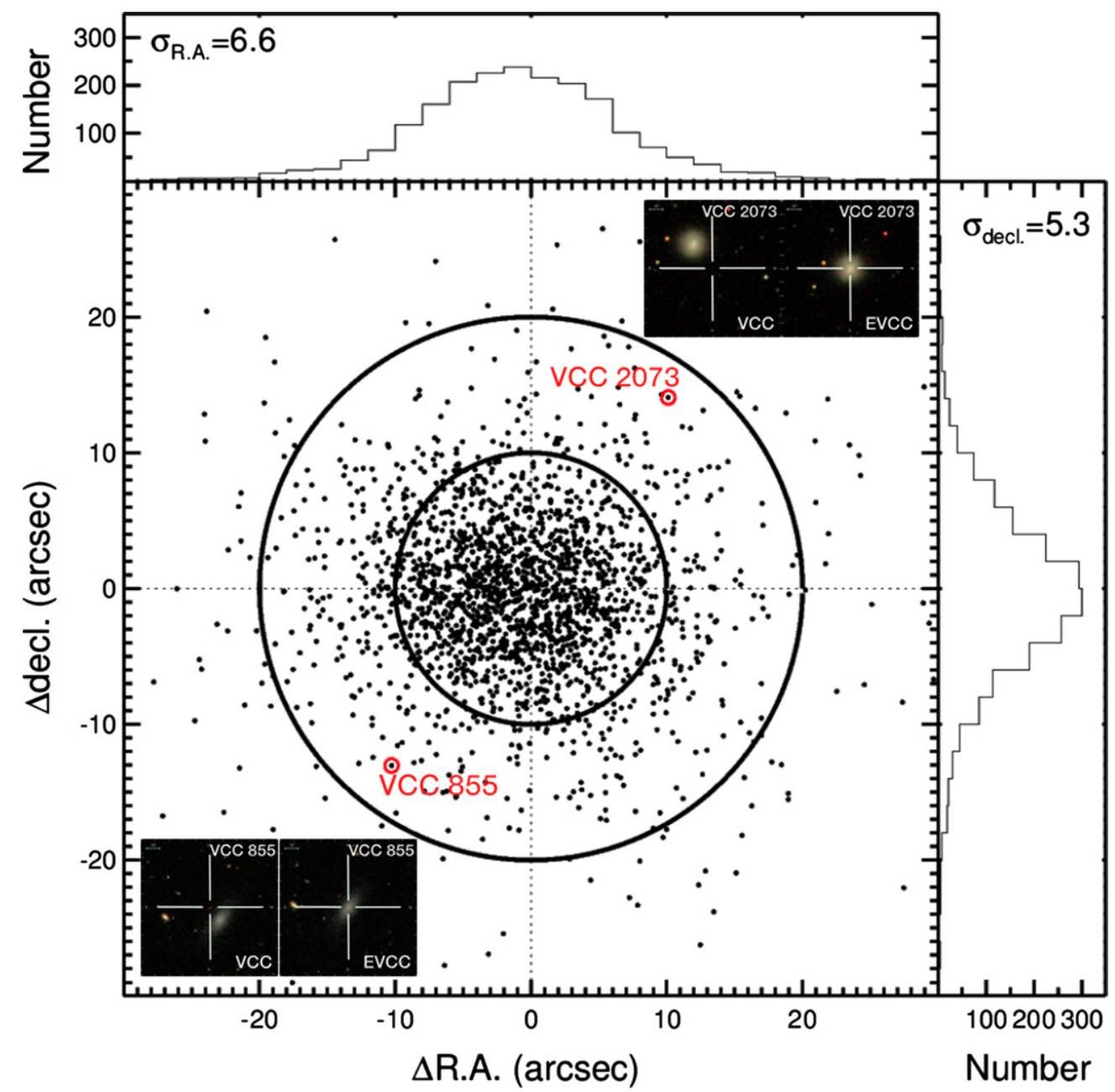

Figure 26. Residual of astrometry between the EVCC and VCC. Two circles of 10 and 20 arcsec radii are superimposed to the plot as references. The histograms are the distributions of the residuals in R.A. (top panel) and decl. (right panel). The standard deviation of the residual distribution (in arcsec) is presented in each panel. Inset images are examples of galaxies (VCC 855 and VCC 2073) with incorrect coordinates in the VCC.

(A color version of this figure is available in the online journal.)

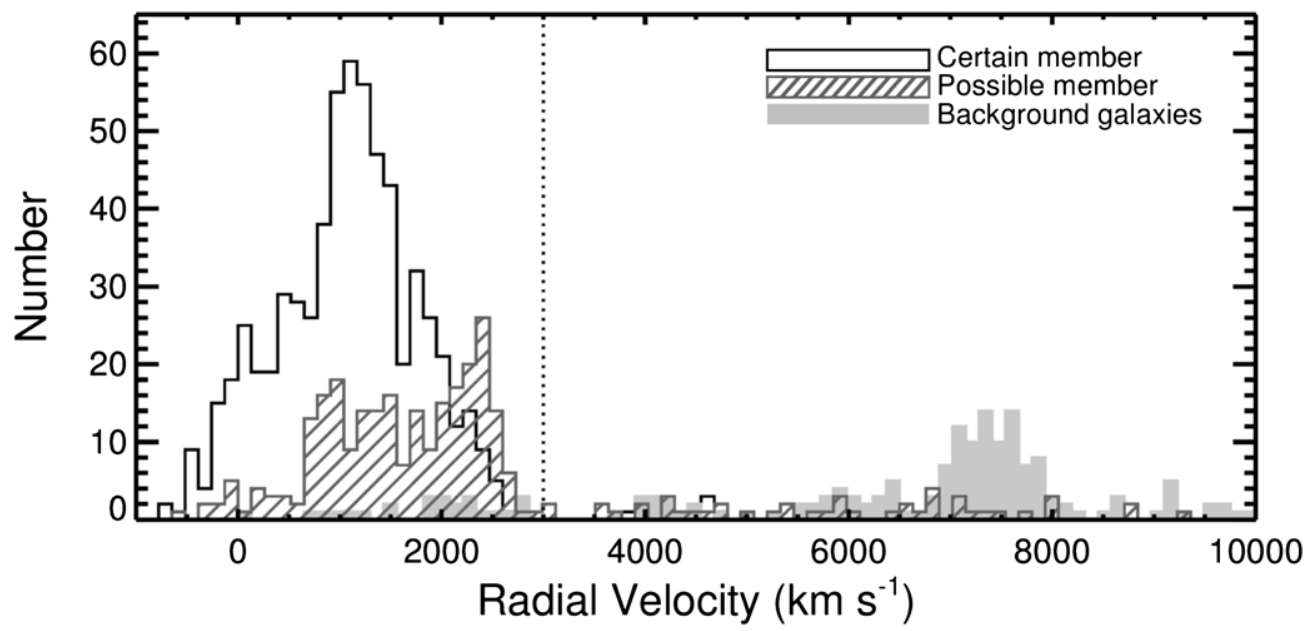

Figure 27. Radial velocity distribution of galaxies in the VCC with available spectra. The solid, hatched, and filled histograms represent certain member, possible member, and background galaxies, respectively, defined by the VCC. The vertical dotted line represents the limit of $3000 \mathrm{~km} \mathrm{~s}^{-1}$.

8. While the EVCC contains galaxies down to about $r=19$, some fainter galaxies in the morphology-based VCC are absent in the EVCC. This is mainly due to the limiting magnitude of SDSS spectroscopic observations and these faint samples are mostly $\mathrm{dE}$ and Irr galaxies. For a given galaxy morphological type except $\mathrm{dE}$ and Irr, the shape of the luminosity function of the EVCC is similar to that of the VCC. 


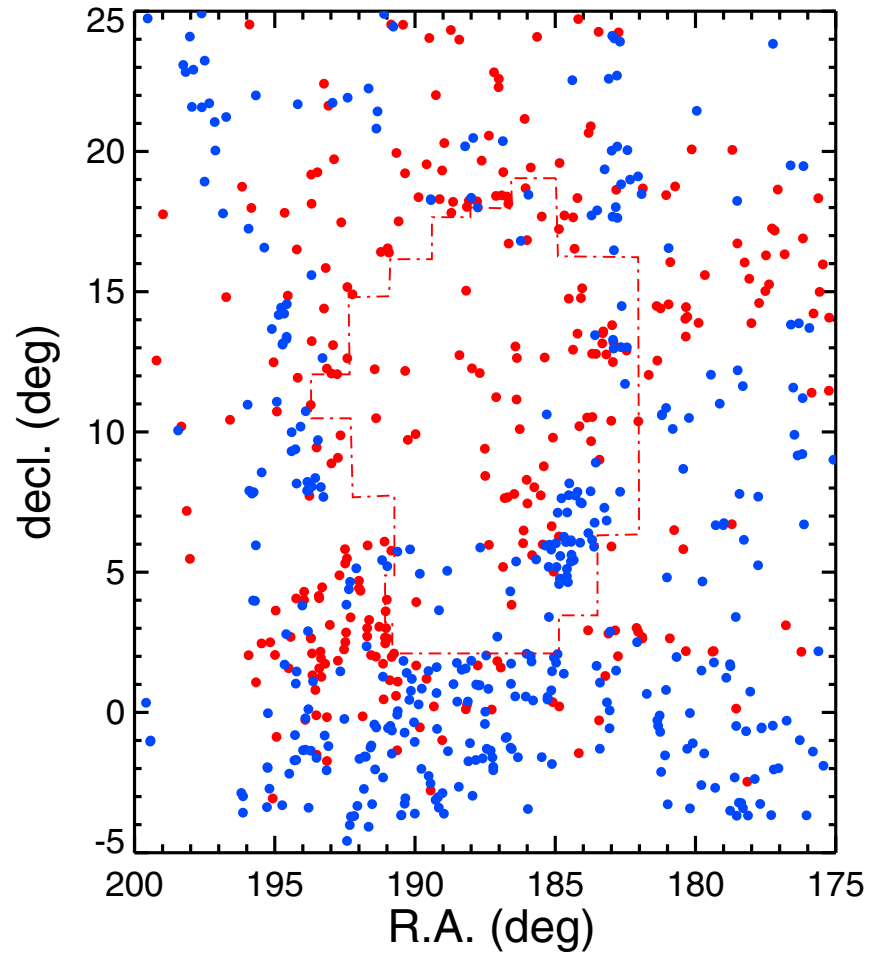

Figure 28. Projected spatial distribution of 676 galaxies included only in the EVCC. The red and blue filled circles are certain and possible member galaxies, respectively. The red contour marks the VCC footprint.

(A color version of this figure is available in the online journal.)

9. We have found that coordinates of large numbers of galaxies in the VCC significantly are deviated from our coordinates and are discrepant from true centers of galaxies. About $28 \%$ of galaxies in the VCC turned out to have different coordinates from the EVCC with offset larger than 10 arcsec.
10. Based on the limit of the radial velocity of $3000 \mathrm{~km} \mathrm{~s}^{-1}$ as a membership criterion in the EVCC, about $12 \%$ and $27 \%$ for certain and possible member galaxies, respectively, in the VCC have larger velocities than $3000 \mathrm{~km} \mathrm{~s}^{-1}$. On the other hand, about $10 \%$ of background galaxies in the VCC are within this radial velocity limit. About $19 \%$ of 676 newly covered galaxies in the EVCC are located inside of the VCC region, while they were not selected as member galaxies in the VCC. These galaxies have faint luminosities and are smaller in size compared to the galaxies in the VCC. They show morphologies of Irr and dE/dSO.

Our EVCC not only updates and extends the VCC, but also will be complementary to other available or ongoing surveys of the Virgo cluster at various wavelengths (see Ferrarese et al. 2012 for a review), and serves as basis for forthcoming scientific studies of the Virgo cluster. Taking advantage of the EVCC catalog in terms of homogeneous data for unprecedented widefield region of the Virgo cluster as well as available radial velocity and spectroscopic information of all member galaxies, our forthcoming papers will explore a number of scientific issues, including the following.

1. Kinematic structure of the Virgo cluster and its dependence on various environmental parameters.

2. Variation of luminosity functions of galaxies in various regions.

3. Chemical properties of extensive emission line galaxies as a function of various cluster environments.

4. Ultraviolet properties of galaxies and correlations with environments.

5. Surface brightness profiles and structural parameters of galaxies.

6. Identification of potential "feeding channels" of the Virgo cluster by using dwarf galaxy properties to relate known cluster substructures to regions beyond the virial radius.

7. Three-dimensional structure of the Virgo environment from surface brightness fluctuations for early-type galaxies.

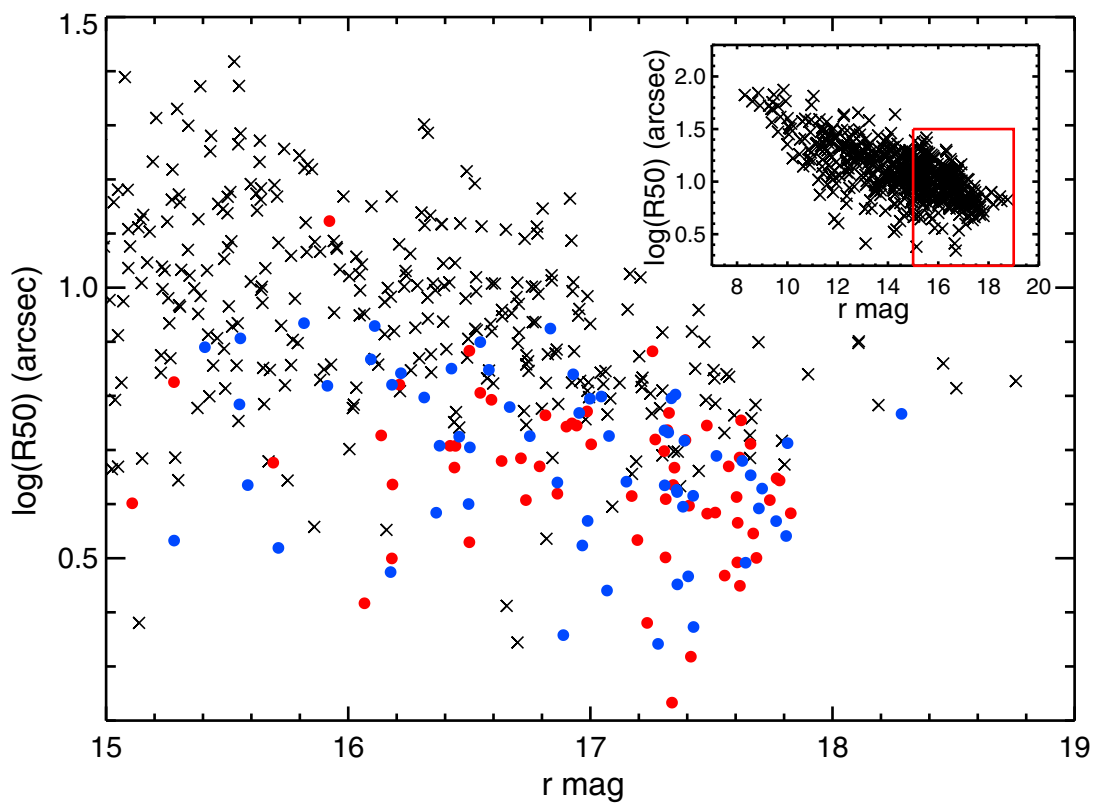

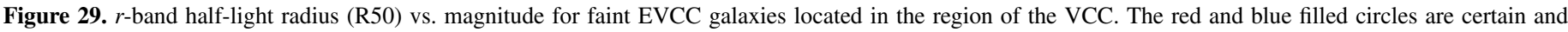

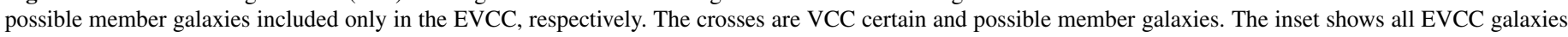
of entire magnitude range located in the $\mathrm{VCC}$ region.

(A color version of this figure is available in the online journal.) 
We are grateful to the anonymous referee for helpful comments and suggestions that improved the clarity and quality of this paper. This research was supported by Basic Science Research Program through the National Research Foundation of Korea (NRF) funded by the Ministry of Education, Science, and Technology (NRF-2012R1A1B4003097). Support for this work was also provided by the NRF of Korea to the Center for Galaxy Evolution Research (No. 2010-0027910). S.K. acknowledges support from the National Junior Research Fellowship of NRF (No. 2011-0012618). T.L. was supported within the framework of the Excellence Initiative by the German Research Foundation (DFG) through the Heidelberg Graduate School of Fundamental Physics (grant number GSC 129/1). Y.L. was supported by the Korea Astronomy and Space Science Institute under the R\&D program (Project No. 2014-1-600-05) supervised by the Ministry of Science, ICT and Future Planning.

\section{REFERENCES}

Abazajian, K., Adelman-McCarthy, J. K., Agüeros, M. A., et al. 2004, AJ, 128,502

Abazajian, K. N., Adelman-McCarthy, J. K., Agüeros, M. A., et al. 2009, ApJS, 182,543

Abdullah, M. H., Ali, G. B., Ismail, H. A., \& Rassem, M. A. 2011, MNRAS, 416, 2027

Aguerri, J. A. L., Gerhard, O. E., Arnaboldi, M., et al. 2005, AJ, 129, 2585

Arnaboldi, M., Gerhard, O., Aguerri, J. A. L., et al. 2004, ApJL, 614, L33

Bertin, E., \& Arnouts, S. 1996, A\&AS, 117, 393

Binggeli, B., \& Cameron, L. M. 1991, A\&A, 252, 27

Binggeli, B., Popescu, C. C., \& Tammann, G. A. 1993, A\&AS, 98, 275

Binggeli, B., Sandage, A., \& Tammann, G. A. 1985, AJ, 90, 1681

Binggeli, B., Sandage, A., \& Tammann, G. A. 1988, ARA\&A, 26, 509

Binggeli, B., Tammann, G. A., \& Sandage, A. 1987, AJ, 94, 251

Blanton, M. R., Kazin, E., Muna, D., Weaver, B. A., \& Price-Whelan, A. 2011, AJ, 142, 31

Blanton, M. R., Lupton, R. H., Schlegel, D. J., et al. 2005a, ApJ, 631, 208

Blanton, M. R., Schlegel, D. J., Strauss, M. A., et al. 2005b, AJ, 129, 2562

Bond, J. R., Kofman, L., \& Pogosyan, D. 1996, Natur, 380, 603

Boselli, A., Boissier, S., Cortese, L., \& Gavazzi, G. 2008, A\&A, 489, 1015

Brough, S., Forbes, D. A., Kilborn, V. A., \& Couch, W. 2006, MNRAS, 370,1223

Bruzual, G., \& Charlot, S. 2003, MNRAS, 344, 1000

Buta, R. J. 2013, Galaxy Morphology, ed. T. D. Oswalt \& W. C. Keel (Berlin: Springer-Verlag), 1

Cardelli, J. A., Clayton, G. C., \& Mathis, J. S. 1989, ApJ, 345, 245

Davies, J. I., Bianchi, S., Baes, M., et al. 2014, MNRAS, 438, 1922

de Vaucouleurs, A., \& de Vaucouleurs, G. 1973, AJ, 78, 377

de Vaucouleurs, G. 1961, ApJS, 6, 213
Ferguson, H. C. 1989, AJ, 98, 367

Ferguson, H. C., \& Sandage, A. 1988, AJ, 96, 1520

Ferrarese, L., Côté, P., Cuillandre, J.-C., et al. 2012, ApJS, 200, 4

Fouque, P., Le Bertre, T., Epchtein, N., Guglielmo, F., \& Kerschbaum, F. 1992, A\&AS, 93, 151

Ftaclas, C., Struble, M. F., \& Fanelli, M. N. 1984, ApJ, 282, 19

Garcia, A. M. 1993, A\&AS, 100, 47

Gavazzi, G., Boselli, A., Scodeggio, M., Pierini, D., \& Belsole, E. 1999, MNRAS, 304, 595

Giuricin, G., Marinoni, C., Ceriani, L., \& Pisani, A. 2000, ApJ, 543, 178

Godwin, J. G., Metcalfe, N., \& Peach, J. V. 1983, MNRAS, 202, 113

Hoffman, G. L., Lewis, B. M., \& Salpeter, E. E. 1995, ApJ, 441, 28

Hoffman, G. L., Olson, D. W., \& Salpeter, E. E. 1980, ApJ, 242, 861

Jerjen, H., Binggeli, B., \& Barazza, F. D. 2004, AJ, 127, 771

Jerjen, H., \& Dressler, A. 1997, A\&AS, 124, 1

Jerjen, H., \& Tammann, G. A. 1997, A\&A, 321, 713

Jing, Y. P., Mo, H. J., Borner, G., \& Fang, L. Z. 1995, MNRAS, 276, 417

Karachentsev, I. D., \& Nasonova, O. G. 2010, MNRAS, 405, 1075

Kennicutt, R. C., Jr. 1992, ApJS, 79, 255

Lisker, T., Glatt, K., Westera, P., \& Grebel, E. K. 2006, AJ, 132, 2432

Lisker, T., Grebel, E. K., \& Binggeli, B. 2008, AJ, 135, 380

Lupton, R. H., Gunn, J. E., \& Szalay, A. S. 1999, AJ, 118, 1406

Makarov, D., \& Karachentsev, I. 2011, MNRAS, 412, 2498

McIntosh, D. H., Guo, Y., Hertzberg, J., et al. 2008, MNRAS, 388, 1537

Mei, S., Blakeslee, J. P., Côté, P., et al. 2007, ApJ, 655, 144

Nolthenius, R. 1993, ApJS, 85, 1

Pak, M., Rey, S.-C., Lisker, T., et al. 2014, MNRAS, 445, 630

Praton, E. A., \& Schneider, S. E. 1994, ApJ, 422, 46

Sánchez Almeida, J., Aguerri, J. A. L., Muñoz-Tuñón, C., \& de Vicente, A. 2010, ApJ, 714, 487

Sánchez Almeida, J., Aguerri, J. A. L., Muñoz-Tuñón, C., \& Huertas-Company, M. 2011, ApJ, 735, 125

Sandage, A., \& Bedke, J. 1994, The Carnegie Atlas of Galaxies. Volumes I, II (Washinton, DC: Carnegie Institution of Washington, D.C.)

Sandage, A., \& Binggeli, B. 1984, AJ, 89, 919

Sandage, A., Binggeli, B., \& Tammann, G. A. 1985a, AJ, 90, 1759

Sandage, A., Binggeli, B., \& Tammann, G. A. 1985b, AJ, 90, 395

Sandage, A., \& Tammann, G. A. 1981, A Revised Shapley-Ames Catalog of Bright Galaxies

Schlegel, D. J., Finkbeiner, D. P., \& Davis, M. 1998, ApJ, 500, 525

Springel, V., White, S. D. M., Jenkins, A., et al. 2005, Natur, 435, 629

Stoughton, C., Lupton, R. H., Bernardi, M., et al. 2002, AJ, 123, 485

Strauss, M. A., Weinberg, D. H., Lupton, R. H., et al. 2002, AJ, 124, 1810

Tully, R. B. 1982, ApJ, 257, 389

Tully, R. B., \& Shaya, E. J. 1984, ApJ, 281, 31

van den Bergh, S. 1960, ApJ, 131, 215

van Haarlem, M., \& van de Weygaert, R. 1993, ApJ, 418, 544

West, A. A., Garcia-Appadoo, D. A., Dalcanton, J. J., et al. 2010, AJ, 139,315

Yasuda, N., Fukugita, M., \& Okamura, S. 1997, ApJS, 108, 417

Yoon, J. H., Putman, M. E., Thom, C., Chen, H.-W., \& Bryan, G. L. 2012, ApJ, 754, 84 